hep-th/0502076

UCB-PTH-04/37

LBNL-56763

\title{
Massless and Massive Three Dimensional Super Yang-Mills Theory and Mini-Twistor String Theory
}

\author{
Dah-Wei Chiou, Ori J. Ganor, Yoon Pyo Hong, Bom Soo Kim and Indrajit Mitra \\ Department of Physics, University of California, Berkeley, CA 94720 \\ and \\ Theoretical Physics Group, Lawrence Berkeley National Laboratory, Berkeley, CA 94720 \\ E-mails: dwchiou, origa, yph, bskim, imitra@socrates . berkeley.edu,
}

\begin{abstract}
We propose various ways of adding mass terms to three-dimensional twistor string theory. We begin with a review of mini-twistor space - the reduction of $D=4$ twistor space to $D=3$. We adapt the two proposals for twistor string theory, Witten's and Berkovits's, to $D=3$ super YangMills theory. In Berkovits's model, we identify the enhanced R-symmetry. We then construct B-model topological string theories that, we propose, correspond to $D=3$ Yang-Mills theory with massive spinors and massive and massless scalars in the adjoint representation of the gauge group. We also analyze the counterparts of these constructions in Berkovits's model. Some of our constructions can be lifted to $D=4$, where infinitesimal mass terms correspond to VEVs of certain superconformal gravity fields.
\end{abstract}




\section{DISCLAIMER}

This document was prepared as an account of work sponsored by the United States Government. While this document is believed to contain correct information, neither the United States Government nor any agency thereof, nor The Regents of the University of California, nor any of their employees, makes any warranty, express or implied, or assumes any legal responsibility for the accuracy, completeness, or usefulness of any information, apparatus, product, or process disclosed, or represents that its use would not infringe privately owned rights. Reference herein to any specific commercial product, process, or service by its trade name, trademark, manufacturer, or otherwise, does not necessarily constitute or imply its endorsement, recommendation, or favoring by the United States Government or any agency thereof, or The Regents of the University of California. The views and opinions of authors expressed herein do not necessarily state or reflect those of the United States Government or any agency thereof or The Regents of the University of California. 


\section{Contents}

1 Introduction $\quad 3$

2 The (mini-)twistor space of $\mathbb{R}^{3} \quad 6$

2.1 Harmonic functions on $\mathbb{R}^{3} \ldots \ldots \ldots \ldots$

2.2 Identification of mini-twistor space with $T \mathbb{C P}^{1} \ldots \ldots \ldots$

2.3 Geometric picture of mini-twistor space . . . . . . . . . . . . . . . . . . . . . . 9

2.4 The Poincaré group . . . . . . . . . . . . . . . . . . . . . . . . . 10

2.5 Extension to Superspace . . . . . . . . . . . . . . . . . . . . . 11

2.6 The scalar propagator $\ldots \ldots \ldots \ldots$. . . . . . . . . . . . . . . . . . 11

2.7 Minkowski space $\mathbb{R}^{2,1} \ldots \ldots \ldots \ldots \ldots \ldots \ldots \ldots$

3 Dimensional reduction $\quad 15$

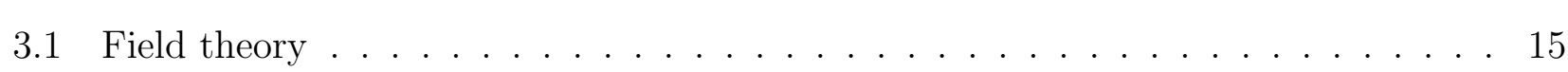

3.2 Dimensional reduction of $D=4$ twistor space . . . . . . . . . . . . . . . . . . 16

3.3 Dimensional reduction of $D=4$ super-twistor space . . . . . . . . . . . . . . . . . 19

3.4 Tree-level amplitudes . . . . . . . . . . . . . . . . . . . . . . . . . . 20

3.5 Explicit examples of amplitudes . . . . . . . . . . . . . . . . . . . . 23

3.6 Phvsical interpretation of the holomorphic curves . . . . . . . . . . . . . . . . 27

3.7 Dimensional reduction in B-model twistor string theorv . . . . . . . . . . . . . . . 29

3.8 Dimensional reduction in Berkovits's twistor string theory . . . . . . . . . . . . . 30

$3.9 \operatorname{Spin}(7)$ R-svmmetrv . . . . . . . . . . . . . . . . . . . . . 33

$3.10 \operatorname{Spin}(7)$ in Berkovits's model . . . . . . . . . . . . . . . . . . . . . . 35

4 Mass terms $\quad 36$

4.1 Dimensional reduction with twists . . . . . . . . . . . . . . . . . . . 37

4.2 Mini-twistor string theorv mass operators . . . . . . . . . . . . . . . . . . . . 38

4.3 The $\mathbf{1 0}$ and $\overline{\mathbf{1 0}}$ mass terms . . . . . . . . . . . . . . . . . . . . . 42

4.4 Chiral $D=4$ mass terms . . . . . . . . . . . . . . . . . . . . . . . . . . . . . . . . 43

4.5 Mass terms in Berkovits's model . . . . . . . . . . . . . . . . . . . . . . . . . . 49

4.6 Application of $\operatorname{Spin}(7) \mathrm{R}$-svmmetrv $\ldots \ldots \ldots \ldots$. . . . . . . . . . . 53

4.7 The singlet mass term . . . . . . . . . . . . . . . . . . . 57 
4.8 Comments on the decoupling limit $M \rightarrow \infty \ldots \ldots \ldots \ldots$

5 Conclusions and discussion $\quad 58$

$\begin{array}{ll}\text { A Dealing with inverses of fields } & 61\end{array}$

\section{Introduction}

Over the last twenty years following the work of Parke and Taylor [1] (among others, see also [2]) it has become clear that the scattering amplitudes of Yang-Mills theory in four dimensions are much simpler than one would guess. This simplicity was known to persist not only for tree-level results, but also at one loop level (for a nice review, see [3]). Witten has recently shown $[4$ that these amplitudes are most succinctly expressed not in terms of the polarization and momenta of the incoming and outgoing photons, but rather in terms of Penrose's twistor variables [5] (which of course encode information about the polarization and momenta of the particles). By applying the so-called twistor transform to the amplitudes expressed in terms of spinor variables, Witten showed that the results collapse to simple algebraic curves in twistor space.

Twistor theory uncovers holomorphic structure underlying massless free field equations of motion. The "twistor transform" converts harmonic functions on a manifold $\mathcal{M}$ to meromorphic functions on its "twistor space" $\mathbb{T} \mathcal{M}$. The twistor transform can be used to convert scattering amplitudes of $n$ massless gluons in perturbative Yang-Mills theory to a meromorphic function (i.e., a section of a certain line bundle) of $n$ points on $\mathbb{T}\left(\mathbb{R}^{4}\right)$. It was conjectured in [4] that the $l$-loop contribution to the transformed amplitude is nonvanishing only if the $n$ points on twistor space that correspond to the $n$ gluons lie on a holomorphic curve whose degree and genus are determined by the helicity of the particles and by $l$. This led Witten to conjecture that there exists a dual string theory and that it is a topological B-model [4]. The target space of this string theory is the twistor space of $\mathbb{R}^{4}$, and Witten showed how the D-instantons of this string theory can compute the scattering amplitudes of the perturbative gauge theory. (The issue of whether one should consider connected or disconnected instantons in order to reproduce the gauge theory amplitudes was rather nicely resolved in [6].) This surprising duality is a "weak-weak" duality in the sense that a weakly coupled string theory is dual to a weakly coupled gauge theory, unlike the "strong-weak" duality [7] [8] 9] where a strongly coupled string theory was dual to a weakly coupled gauge theory. 
We wish to extend these results to three dimensions and to understand the string dual of weakly coupled super Yang-Mills theory in $D=3$. The target space of this string theory is the twistor space of $\mathbb{R}^{3}$. This turns out to be the space of oriented lines in $\mathbb{R}^{3}$ (we mostly follow [10] and the nice review article [11]). It is called "mini-twistor" space [10], and is related to the twistor space of $\mathbb{R}^{3,1}$ by dimensional reduction. This well-known elegant relation [10] will guide us in developing an algorithm to obtain gauge theory scattering amplitudes in $D=3$ from the corresponding ones in $D=4$. We shall see that the $D=3$ amplitudes are still supported on holomorphic curves.

Recall that the twistor space of Minkowski space $\mathbb{R}^{3,1}$ is $\mathbb{T}\left(\mathbb{R}^{3,1}\right) \simeq \mathbb{C P}^{3} \backslash \mathbb{C P}^{1}$ (i.e., $\mathbb{C P}^{3}$ with a rational curve excised ${ }^{1}$ ); the twistor space of $\mathbb{R}^{3}$ is $T \mathbb{C P}^{1}$ (the tangent space of $\mathbb{C P}^{1}$ ) [10] [11. ${ }^{2}$ The mini-twistor space does not possess the full $D=3$ superconformal symmetry $S O(3,2)$, but only its Poincaré subgroup $S O(3) \ltimes \mathbb{R}^{3}$. It can be obtained by dimensional reduction as follows [10]. The 3(complex)-dimensional $D=4$ twistor space $\mathbb{C P}^{3} \backslash \mathbb{C P}^{1}$ can be written as a fiber bundle with the 2(complex)-dimensional $D=3$ mini-twistor space $T \mathbb{C P}^{1}$ as the base, and the fiber is $\mathbb{C}$. The structure group is the additive translation group $\sim \mathbb{C}$ (as opposed to the multiplicative group $\left.\mathbb{C}^{*}\right)$. However, the fibration is not canonical; it depends on a choice of direction in the physical space $\mathbb{R}^{4}$. This is the direction of the dimensional reduction. For a given choice of this direction (which we will refer to as the " $4^{\text {th }}$ direction") there is a natural projection from the $D=4$ twistor space $\mathbb{C P}^{3} \backslash \mathbb{C P}^{1}$ onto the $D=3$ mini-twistor space $T \mathbb{C P}^{1}$. We will use this fibration to calculate mini-twistor amplitudes of $D=3$ Yang-Mills theory by taking the $D=4$ amplitudes and integrating them over the $\mathbb{C}$ fibers of the above fibration. In the worldsheet theory of the $D=4$ twistor string theory - the B-model with target space $\mathbb{C P}^{3 \mid 4}$ —we realize dimensional reduction by gauging one of the four translation symmetries. It is an element of the B-model symmetry group $P S L(4 \mid 4)$. The resulting string theory is the B-model with target space $T \mathbb{C P}^{1}$ and four additional local fermionic coordinates that transform as sections of the pullback of the $\mathcal{O}(1)$ line bundle over $\mathbb{C P}^{1}$.

One aspect that is not so obvious in this construction is the enhanced R-symmetry. The Rsymmetry group of $D=3$ super Yang-Mills with $N=8$ supersymmetry is $\operatorname{Spin}(7)$, but we will find that only an $S U(4)$ subgroup is manifest in the B-model string theory. There is, however,

\footnotetext{
${ }^{1}$ We use the operator $\backslash$ to denote the set-theory "minus" and it is not to be confused with division.

${ }^{2}$ This space has also been discussed in footnote 13 of [4], and in [12] it was derived by dimensional reduction with constraints, from 2-Time physics.
} 
another version of twistor string theory due to Berkovits [13. This is an open string theory. There is a prescription due to Berkovits and Witten [14] which allows one to go from one picture to the other, and we will use this somewhat extensively. We will implement dimensional reduction from $D=4$ to $D=3$ in Berkovits's model as well, and we will derive the $D=3$ version of this string theory. In this string theory we will be able to construct the full R-symmetry current.

We will also describe a twistor string theory dual of a certain massive $D=3$ super Yang-Mills theory, and this is one of our main new results. The target space of this string theory is also $T \mathbb{C P}^{1}$ with four additional local fermionic coordinates, but the way in which they are fibered over $T \mathbb{C P}^{1}$ is modified from the massless case. It corresponds to a Yang-Mills theory with massive scalars and fermions. We will systematically study what adding small mass terms to the fermions means for the string theory. We will study this question both in the context of the B-model and Berkovits's open string theory. In the dimensionally reduced $D=3$ gauge theory, mass terms have two different origins. They either come from mass terms in the original $D=4$ theory, or they come by coupling the R-symmetry current to a constant background gauge field (i.e., an R-symmetry twist).

In the B-model, the effect of a mass term for the space-time fermions can be achieved by deforming the (super-)complex structure of the target space. Such a deformation corresponds to a closed string vertex operator. We will identify the vertex operators which give rise to the mass terms. Then, using the prescription of Berkovits and Witten, we convert these operators to open string operators which deform the boundary of the worldsheet. In making this transformation, we encounter a surprise: The operators that one gets using this dictionary do not lie in an irreducible representation of the R-symmetry group Spin(7), as they should. Some of the operators have to be modified to fit into the required irreducible representation.

We also propose that an infinitesimal mass term in $D=4$ can be achieved by a certain marginal deformation of the worldsheet theory. In physical space, this corresponds to a small VEV for a B-model closed string field, which according to [4] 14] is part of a conformal supergravity multiplet. We identify this field.

The paper is organized as follows. In $₫ 2$ we review the construction of mini-twistor space and its geometrical interpretation as the space of oriented lines, following [10] 11]. We review the relation between harmonic functions on $\mathbb{R}^{3}$ and meromorphic forms on mini-twistor space, and we apply it to the scalar propagator. In 93 we review the connection [10] between the $D=3$ and $D=4$ twistor spaces, and we discuss the supersymmetric extensions. We derive the tree-level 
amplitudes of $D=3$ super Yang-Mills theory by dimensional reduction, and we find that they have support on holomorphic curves, like their $D=4$ counterparts. We comment on a possible physical interpretation of this result. In $\$ 4$ we augment the theory with mass terms, and we relate the infinitesimal mass terms to worldsheet operators in the B-model and in Berkovits's model. We conclude in 95 .

\section{The (mini-)twistor space of $\mathbb{R}^{3}$}

The twistor space of $\mathbb{R}^{3}$ is $T \mathbb{C P}^{1}$ - the tangent bundle of $\mathbb{C P}^{1}$, and harmonic functions on $\mathbb{R}^{3}$ can be converted into meromorphic functions on $T \mathbb{C P}^{1}$. The space $T \mathbb{C P}^{1}$ is called "mini-twistor" space [10]. We will now explain these statements in detail and apply them to convert the propagator of massless fields on $\mathbb{R}^{3}$ to a meromorphic function of two points in mini-twistor space. Our initial discussion is based mostly on the nice review paper by Paul Baird [1].

Our discussion in this section is limited to scalar fields. This is not too much of a restriction, since in $D=3$ massless gauge fields can be converted by duality to massless scalars, as we will review in 3.1. Massless spinors in $D=3$ also have just one helicity state, and solutions to the massless Dirac equation can be readily converted to mini-twistor space. We refer the reader to [1] for further details.

\subsection{Harmonic functions on $\mathbb{R}^{3}$}

Pick coordinates $x_{1}, x_{2}, x_{3}$ on $\mathbb{R}^{3}$. For any fixed $0 \leq \theta \leq 2 \pi$, the linear expression $x_{1}+i x_{2} \sin \theta+$ $i x_{3} \cos \theta$ is a harmonic function, and so is any analytic function of this expression. We can construct a more complicated harmonic function on $\mathbb{R}^{3}$ by taking linear combinations of analytic functions of $x_{1}+i x_{3} \cos \theta+i x_{2} \sin \theta$ for various values of $\theta$. Whittaker's formula states that a complex-valued harmonic function $\phi$ on $\mathbb{R}^{3}$ can be given by an integral

$$
\phi\left(x_{1}, x_{2}, x_{3}\right)=\int_{0}^{2 \pi} d \theta f\left(x_{1}+i x_{2} \sin \theta+i x_{3} \cos \theta, \theta\right),
$$

where $f(z, \theta)$ is analytic in the first variable. To prove this formula, note that the right-hand side of (2.1) is obviously harmonic. In order to write a Whittaker formula for an arbitrary harmonic function $\phi$, pick polar coordinates $(r, u, v)$ such that

$$
x_{1}=r \cos u, \quad x_{2}=r \sin u \sin v, \quad x_{3}=r \sin u \cos v .
$$


Then, for $l \geq 0$, and $|m| \leq l$, the spherical harmonics can be written as

$$
r^{l} Y_{l m}(u, v)=\frac{\sqrt{(2 l+1)(l-m) !(l+m) !}}{4 \pi^{3 / 2} i^{3 m} l !} \int_{0}^{2 \pi} d \theta e^{i m \theta}\left(x_{1}+i x_{2} \sin \theta+i x_{3} \cos \theta\right)^{l},
$$

and later we will also need the identity

$$
\frac{1}{r^{l+1}} Y_{l, m}(u, v)= \pm \frac{i^{3 m} l !}{4 \pi^{3 / 2}} \sqrt{\frac{2 l+1}{(l-m) !(l+m) !}} \int_{0}^{2 \pi} \frac{d \theta e^{i m \theta}}{\left(x_{1}+i x_{2} \sin \theta+i x_{3} \cos \theta\right)^{l+1}}
$$

where the sign on the right-hand side of (2.3) is the same as that of $x_{1}$. The formula (2.2) is a standard integral representation of spherical harmonics, while (2.3) can be derived by starting from $[15$

$$
P_{l}^{m}(\cos u)=\frac{(-1)^{m}}{\pi} \frac{l !}{(l-m) !} e^{m \pi i / 2} \int_{0}^{\pi} \frac{\cos m \theta d \theta}{(\cos u+i \sin u \cos \theta)^{l+1}} \quad(\cos u>0) .
$$

Any harmonic function that is regular at the origin can be expanded as a linear combination of spherical harmonics

$$
\phi\left(x_{1}, x_{2}, x_{3}\right)=\sum_{l=0}^{\infty} \sum_{m=-l}^{l} C_{l m} r^{l} Y_{l m}(u, v) .
$$

This allows us to express $\phi$ as a Whittaker integral (2.1). A possible choice for the analytic function to be used on the right-hand side of (2.1) is

$$
f(\zeta, \theta)=\sum_{l=0}^{\infty} \sum_{m=-l}^{l} C_{l m} \frac{\sqrt{(2 l+1)(l-m) !(l+m) !}}{4 \pi^{3 / 2} i^{3 m} l !} e^{i m \theta} \zeta^{l}
$$

\subsection{Identification of mini-twistor space with $T \mathbb{C P}^{1}$}

As explained in [10] 11], the twistor space of $\mathbb{R}^{3}$ can be identified with the space of oriented lines in $\mathbb{R}^{3}$. This space is isomorphic to the 2-complex dimensional space $T \mathbb{C P}^{1}$. We will now review how this works.

First we rewrite Whittaker's formula (2.1) by introducing complex coordinates

$$
w=2 e^{i \theta}\left(x_{1}+i x_{2} \sin \theta+i x_{3} \cos \theta\right), \quad z=e^{i \theta}
$$

Given the analytic function $f$ on the right-hand side of (2.1), it is convenient to define a related analytic function $\varphi$ by

$$
\varphi\left(e^{i \theta}, w\right):=e^{-i \theta} f\left(\frac{1}{2} e^{-i \theta} w, \theta\right)
$$


We assume that we can extend $\varphi$ to an analytic function $\varphi(z, w)$ defined in a neighborhood of the circle $|z|=1$. Formula (2.1) can now be rewritten as

$$
\phi(\vec{x})=\frac{1}{2 \pi i} \oint \varphi\left(z,-\left[x_{2}-i x_{3}\right]+2 z x_{1}+z^{2}\left[x_{2}+i x_{3}\right]\right) d z .
$$

We take $z$ and $w$ as local coordinates on mini-twistor space, which will be identified with $T \mathbb{C P}^{1}$ soon. Under favorable conditions, $\varphi(z, w)$ can be analytically continued to a meromorphic function for all $z \in \mathbb{C}$ and $w \in \mathbb{C}$. For simplicity of the discussion we will assume that this is the case. (Although, in the more general case we can assume that $\varphi$ can be analytically continued to a neighborhood around $|z|=1$. We defer the discussion of this case till the end of this subsection, since we will need to use sheaf cohomology.) We would actually like to view $z$ as a coordinate on a $\mathbb{C P}^{1}$ by identifying $\mathbb{C P}^{1} \simeq \mathbb{C} \cup\{\infty\}$ (say, by stereographic projection), so that $z=\infty$ will be an allowed value. Then, for every $\vec{x} \in \mathbb{R}^{3}$, the equation

$$
w=-\left(x_{2}-i x_{3}\right)+2 x_{1} z+\left(x_{2}+i x_{3}\right) z^{2} \quad \text { (incidence relation) }
$$

which is analytic in $z$, defines an algebraic curve in $(z, w)$ space. We get the integrand of (2.4) from $\varphi(z, w)$ by setting (2.5) . The right-hand side of (2.5) has a double pole at $z=\infty$. We define

$$
z^{\prime}=\frac{1}{z}, \quad w^{\prime}=-\frac{w}{z^{2}}
$$

to be regular local coordinates on mini-twistor space near $z=\infty$, instead of $(z, w)$. Equation (2.5) then becomes

$$
w^{\prime}=-\left(x_{2}+i x_{3}\right)-2 x_{1} z^{\prime}+\left(x_{2}-i x_{3}\right) z^{\prime 2}
$$

which has no pole at $z=\infty$. The two coordinate systems $(z, w)[z \neq \infty]$ and $\left(z^{\prime}, w^{\prime}\right)\left[z^{\prime} \neq \infty\right]$, with the transition rules (2.6), describe the holomorphic line bundle $\mathcal{O}(2)$ over $\mathbb{C P}^{1}, w$ being the local coordinate on the fiber and $z$ being the coordinate on the base. $\mathcal{O}(2)$ can be identified with the tangent bundle $T \mathbb{C P}^{1}$, since from (2.6) it is obvious that $w$ transforms like a vector on $\mathbb{C P}^{1}$. Thus we identify the mini-twistor space $\mathbb{T}\left(\mathbb{R}^{3}\right)$ with $T \mathbb{C P}$.

The relation (2.5) describes a holomorphic section of the line bundle $T \mathbb{C P}^{1}$, and it varies with the point $\vec{x} \in \mathbb{R}^{3}$. It is called the incidence relation [11]. In 92.3 , following [11, we will give the mini-twistor space and the incidence relation a more geometric interpretation.

From (2.4) we see that it is natural to think of $\varphi$ as a holomorphic 1 -form $\varphi(z, w) d z$. This 1 -form is defined in the neighborhood of $|z|=1$. A 1 -form on $\mathbb{C P}^{1}$, by definition, takes values 


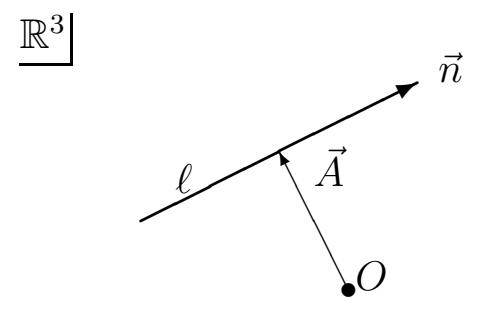

Figure 1: Mini-twistor space can be identified with the space of oriented lines $\ell \in \mathbb{R}^{3}$. The direction of $\ell$ is $\vec{n}$, and the displacement vector is $\vec{A}$.

in the holomorphic sheaf $\Omega^{1} \simeq \mathcal{O}(-2)$ over $\mathbb{C P}^{1}$. We can think of $\varphi d z$ as taking values in the pullback $\widetilde{\Omega}^{1}$ of $\Omega^{1}$ to the tangent bundle $T \mathbb{C P}^{1}$. Also, the integral (2.4) is unchanged if we replace $\varphi$ with $\varphi(z, w) d z+g_{0}(z, w) d z-g_{\infty}(z, w) d z$, for any pair of local holomorphic 1-forms $g_{0}$ and $g_{\infty}$ (taking values in the sheaf $\widetilde{\Omega}^{1}$, by definition) such that $g_{0}$ has no poles for all $|z| \leq 1$ and $g_{\infty}$ has no poles for all $|z| \geq 1$ (including $z=\infty$ ). This defines an equivalence class of 1-forms $\varphi \sim \varphi+g_{0}-g_{\infty}$, which defines the sheaf cohomology $H^{1}\left(T \mathbb{C P}^{1}, \widetilde{\Omega}^{1}\right)$. This notion will be useful in 3.4 when we derive mini-twistor tree-level amplitudes of $D=3$ super Yang-Mills theory by dimensional reduction of $D=4$ amplitudes.

\subsection{Geometric picture of mini-twistor space}

As explained in [10] [1], the mini-twistor space $T \mathbb{C P}^{1}$, whose construction was reviewed above, can be identified with the space of oriented lines in $\mathbb{R}^{3}$, and the incidence relation (2.5) is the condition that the line that corresponds to the mini-twistor $(z, w)$ passes through $\vec{x} \in \mathbb{R}^{3}$. We will now review how this works.

To describe an oriented line $\ell$ in $\mathbb{R}^{3}$, we need its direction, which is a unit vector $\vec{n}$, and its displacement vector $\vec{A}$, which is the vector from the origin of $\mathbb{R}^{3}$ to a point on $\ell$ closest to the origin. Therefore, $\vec{A}$ is perpendicular to $\vec{n}$. The space of unit vectors $\vec{n}$ in $\mathbb{R}^{3}$ is the sphere $S^{2}$, which we identify with $\mathbb{C P}^{1}$ by the stereographic projection:

$$
\|\vec{n}\|=1 \Longrightarrow z=\frac{n^{2}-i n^{3}}{1+n^{1}} \in \mathbb{C P}^{1} \simeq \mathbb{C} \cup\{\infty\}
$$

Given $\vec{n}$, the space of vectors $\vec{A}$ that satisfy $\vec{n} \cdot \vec{A}=0$ is the tangent plane to $\vec{n}$ on $S^{2}$. With the identification $S^{2} \simeq \mathbb{C P}^{1}$ we find that the space of $(\vec{n}, \vec{A})$ is $T \mathbb{C P}^{1}$. The holomorphic coordinate $w$ 
on the fiber of $T \mathbb{C P} P^{1}$ can be defined as

$$
w=-A^{k} \frac{\partial z}{\partial n^{k}}=\frac{-\left(1+n^{1}\right)\left(A^{2}-i A^{3}\right)+\left(n^{2}-i n^{3}\right) A^{1}}{\left(1+n^{1}\right)^{2}},
$$

where $A^{i}$ and $n^{i}(i=1,2,3)$ are the components of $\vec{A}$ and $\vec{n}$ respectively. Given $z$, we can recover $\vec{n}$ by

$$
\vec{n}=\left(\frac{1-|z|^{2}}{1+|z|^{2}}, \frac{z+\bar{z}}{1+|z|^{2}}, \frac{i(z-\bar{z})}{1+|z|^{2}}\right)
$$

and, noting that $\vec{n} \cdot \vec{A}=0$, we can recover $\vec{A}$ from $(z, w)$ :

$$
\vec{A}=\left(2 \frac{w \bar{z}+\bar{w} z}{\left(1+|z|^{2}\right)^{2}},-\frac{w\left(1-\bar{z}^{2}\right)+\bar{w}\left(1-z^{2}\right)}{\left(1+|z|^{2}\right)^{2}},-i \frac{w\left(1+\bar{z}^{2}\right)-\bar{w}\left(1+z^{2}\right)}{\left(1+|z|^{2}\right)^{2}}\right) .
$$

One can check [1] that the oriented line given by $\vec{x}=\left(x_{1}, x_{2}, x_{3}\right)=\vec{A}+c \vec{n}$ is the solution set to the incidence relation (2.5).

\subsection{The Poincaré group}

It will be useful for us to express the generators of the symmetry group of $T \mathbb{C P}^{1}$ in mini-twistor variables. This will allow us to easily check symmetry properties of various expressions that are given in terms of $z$ and $w$. The Poincaré group in three dimensions has generators $\vec{P}$ (translations) and $\vec{J}$ (rotations). It acts on $\vec{n}$ and $\vec{A}$ as follows:

$$
\left[P_{i}, n_{j}\right]=0, \quad\left[P_{i}, A_{j}\right]=i \delta_{i j}-i n_{i} n_{j}, \quad\left[J_{i}, n_{j}\right]=i \epsilon_{i j k} n_{k}, \quad\left[J_{i}, A_{j}\right]=i \epsilon_{i j k} A_{k}
$$

From this and (2.8)-(2.9) it is easy to find the expressions in terms of $z$ and $w$. We get

$$
\begin{aligned}
P_{1} & =i z \frac{\partial}{\partial w}+i \bar{z} \frac{\partial}{\partial \bar{w}} \\
P_{+} & :=P_{2}+i P_{3}=-i \frac{\partial}{\partial w}+i \bar{z}^{2} \frac{\partial}{\partial \bar{w}} \\
P_{-} & :=P_{2}-i P_{3}=i z^{2} \frac{\partial}{\partial w}-i \frac{\partial}{\partial \bar{w}},
\end{aligned}
$$

and

$$
\begin{aligned}
J_{1} & =-z \frac{\partial}{\partial z}+\bar{z} \frac{\partial}{\partial \bar{z}}-w \frac{\partial}{\partial w}+\bar{w} \frac{\partial}{\partial \bar{w}} \\
J_{+} & :=J_{2}+i J_{3}=\frac{\partial}{\partial z}+\bar{z}^{2} \frac{\partial}{\partial \bar{z}}+2 \overline{z w} \frac{\partial}{\partial \bar{w}} \\
J_{-} & :=J_{2}-i J_{3}=-z^{2} \frac{\partial}{\partial z}-\frac{\partial}{\partial \bar{z}}-2 z w \frac{\partial}{\partial w}
\end{aligned}
$$




\subsection{Extension to Superspace}

We can easily extend the discussion to accommodate supersymmetry. For $D=3, N=2$ supersymmetry, the generators are $Q_{ \pm}$and their hermitian conjugates are $Q_{ \pm}^{\dagger} \equiv \bar{Q}_{\mp}$. The SUSY algebra is

$$
\begin{gathered}
{\left[P_{i}, Q_{ \pm}\right]=0, \quad\left[P_{i}, \bar{Q}_{ \pm}\right]=0, \quad\left[J_{1}, Q_{ \pm}\right]= \pm \frac{1}{2} Q_{ \pm}, \quad\left[J_{1}, \bar{Q}_{ \pm}\right]= \pm \frac{1}{2} Q_{ \pm}} \\
{\left[J_{ \pm}, Q_{\mp}\right]=Q_{ \pm}, \quad\left[J_{ \pm}, \bar{Q}_{\mp}\right]=-\bar{Q}_{ \pm}, \quad\left[J_{ \pm}, Q_{ \pm}\right]=0, \quad\left[J_{ \pm}, \bar{Q}_{ \pm}\right]=0,}
\end{gathered}
$$

and

$$
\left\{Q_{ \pm}, Q_{ \pm}\right\}=\left\{Q_{ \pm}, Q_{\mp}\right\}=\left\{\bar{Q}_{ \pm}, \bar{Q}_{\mp}\right\}=\left\{\bar{Q}_{ \pm}, \bar{Q}_{ \pm}\right\}=0
$$

and

$$
\left\{Q_{ \pm}, \bar{Q}_{ \pm}\right\}=P_{ \pm}, \quad\left\{Q_{ \pm}, \bar{Q}_{\mp}\right\}= \pm P_{1} .
$$

We add a superspace coordinate $\theta$ and its complex conjugate $\bar{\theta}$. We can now express $Q_{ \pm}$and $\bar{Q}_{ \pm}$ in terms of $(z, w, \theta)$ and their conjugates $(\bar{z}, \bar{w}, \bar{\theta})$ :

$$
\begin{array}{ll}
Q_{+}=\frac{\partial}{\partial \theta}+i \bar{\theta} \bar{z} \frac{\partial}{\partial \bar{w}}, & \bar{Q}_{+}=\bar{z} \frac{\partial}{\partial \bar{\theta}}-i \theta \frac{\partial}{\partial w}, \\
Q_{-}=z \frac{\partial}{\partial \theta}-i \bar{\theta} \frac{\partial}{\partial \bar{w}}, & \bar{Q}_{-}=\frac{\partial}{\partial \bar{\theta}}+i \theta z \frac{\partial}{\partial w} .
\end{array}
$$

The angular momentum operators change slightly with the fermionic contributions:

$$
\begin{aligned}
& J_{1}=-z \frac{\partial}{\partial z}+\bar{z} \frac{\partial}{\partial \bar{z}}-w \frac{\partial}{\partial w}+\bar{w} \frac{\partial}{\partial \bar{w}}-\frac{1}{2} \theta \frac{\partial}{\partial \theta}+\frac{1}{2} \bar{\theta} \frac{\partial}{\partial \bar{\theta}}, \\
& J_{+} \equiv J_{2}+i J_{3}=\frac{\partial}{\partial z}+\bar{z}^{2} \frac{\partial}{\partial \bar{z}}+2 \overline{z w} \frac{\partial}{\partial \bar{w}}+\bar{z} \bar{\theta} \frac{\partial}{\partial \bar{\theta}} \\
& J_{-} \equiv J_{2}-i J_{3}=-z^{2} \frac{\partial}{\partial z}-\frac{\partial}{\partial \bar{z}}-2 z w \frac{\partial}{\partial w}-z \theta \frac{\partial}{\partial \theta} .
\end{aligned}
$$

Note that $\bar{z}$ and $\bar{w}$ have $J_{1}$-eigenvalue of $+1, \bar{\theta}$ and $Q_{+}, \bar{Q}_{+}$have $J_{1}$-eigenvalue $+\frac{1}{2}, \theta$ and $Q_{-}, \bar{Q}_{-}$ have $J_{1}$-eigenvalue $-\frac{1}{2}$, and $z, w$ have $J_{1}$-eigenvalue -1 . For $N=8$ supersymmetry, we take four copies of the fermionic coordinates $\theta^{A}, \bar{\theta}^{A}(A=1, \ldots, 4)$. We will denote this super mini-twistor space by $\mathbb{T}_{3}$. It is $T \mathbb{C P}^{1}$ with the four fermionic coordinates $\theta^{A}$ fibered over it.

\subsection{The scalar propagator}

For a fixed point $\vec{x}^{\prime} \in \mathbb{R}^{3}$ the Green's function for Laplace's equation on $\mathbb{R}^{3}$,

$$
G\left(\vec{x}, \vec{x}^{\prime}\right)=\frac{1}{\left\|\vec{x}-\vec{x}^{\prime}\right\|},
$$


is harmonic away from $\vec{x}=\vec{x}^{\prime}$, and therefore it should be possible to express it as in Whittaker's formula (2.4). In fact, it is not hard to check that

$$
\frac{1}{\left\|\vec{x}-\vec{x}^{\prime}\right\|} \operatorname{sgn}\left(x_{1}^{\prime}-x_{1}\right)=\frac{1}{2 \pi i} \oint \varphi\left(z,-\left[x_{2}-i x_{3}\right]+2 z x_{1}+z^{2}\left[x_{2}+i x_{3}\right] ; \vec{x}^{\prime}\right) d z,
$$

where

$$
\varphi\left(z, w ; \vec{x}^{\prime}\right)=\frac{2}{\left(x_{2}^{\prime}+i x_{3}^{\prime}\right) z^{2}+2 x_{1}^{\prime} z-\left(x_{2}^{\prime}-i x_{3}^{\prime}\right)-w} .
$$

Note that $\varphi$ has a simple pole whenever the mini-twistor $(z, w)$ and the point $\vec{x}^{\prime}$ satisfy the incidence relation (2.5). Thus, the integral (2.4) diverges when $\vec{x}=\vec{x}^{\prime}$, as it should. The extra $\operatorname{sign} \operatorname{sgn}\left(x_{1}^{\prime}-x_{1}\right)$ on the left-hand side is required if we take the contour of integration in (2.4) to be the unit circle $|z|=1$.

The left-hand side of (2.14) is translationally invariant. Likewise, the mini-twistor transform $\varphi\left(z, w ; \vec{x}^{\prime}\right)$ is also translationally invariant in the sense that it satisfies

$$
\begin{aligned}
0 & =\left(\frac{\partial}{\partial w}-\frac{1}{2} \frac{\partial}{\partial x_{2}^{\prime}}-\frac{i}{2} \frac{\partial}{\partial x_{3}^{\prime}}\right) \varphi\left(z, w ; \vec{x}^{\prime}\right), \\
0 & =\left(z \frac{\partial}{\partial w}+\frac{1}{2} \frac{\partial}{\partial x_{1}^{\prime}}\right) \varphi\left(z, w ; \vec{x}^{\prime}\right), \\
0 & =\left(-z^{2} \frac{\partial}{\partial w}-\frac{1}{2} \frac{\partial}{\partial x_{2}^{\prime}}+\frac{i}{2} \frac{\partial}{\partial x_{3}^{\prime}}\right) \varphi\left(z, w ; \vec{x}^{\prime}\right) .
\end{aligned}
$$

Here we have used (2.10) to write the translation generators in terms of $z, w$. The translational invariance of $\varphi\left(z, w ; \vec{x}^{\prime}\right)$ is not a completely trivial statement, because the integral on the righthand side of (2.14) would have been translationally invariant even if, say, the left-hand sides of (2.16) were not zero but were holomorphic functions of $z, w$.

Can we go one step further and mini-twistor transform $\varphi\left(z, w ; \vec{x}^{\prime}\right)$ with respect to $\vec{x}^{\prime}$ to get a meromorphic function of two mini-twistors $(z, w)$ and $\left(z^{\prime}, w^{\prime}\right)$ ? Using the familiar expansion

$$
\frac{1}{\left\|\vec{x}-\vec{x}^{\prime}\right\|}=4 \pi \sum_{l=0}^{\infty} \sum_{m=-l}^{l} \frac{1}{2 l+1} \frac{\|\vec{x}\|^{l}}{\left\|\vec{x}^{\prime}\right\|^{l+1}} Y_{l m}\left(\frac{\vec{x}}{\|\vec{x}\|}\right) Y_{l m}^{*}\left(\frac{\vec{x}^{\prime}}{\left\|\vec{x}^{\prime}\right\|}\right) \quad\left(\text { for }\|\vec{x}\|<\left\|\vec{x}^{\prime}\right\|\right)
$$

and the mini-twistor transforms (2.2)-(2.3), we arrive at the mini-twistor transform of the scalar propagator

$$
\widetilde{G}\left(\mathfrak{t}, \mathfrak{t}^{\prime}\right) \equiv \widetilde{G}\left(z, w ; z^{\prime}, w^{\prime}\right)=\frac{2\left(w^{\prime} z+w z^{\prime}\right)}{\left(w-w^{\prime}\right)\left(w z^{\prime 2}-w^{\prime} z^{2}\right)},
$$

where $\mathfrak{t} \equiv(z, w)$ and $\mathfrak{t}^{\prime} \equiv\left(z^{\prime}, w^{\prime}\right)$ are shorthand for our twistor variables. 
It can be explicitly checked that if $\|\vec{x}\|<\left\|\vec{x}^{\prime}\right\|$, then

$$
\begin{aligned}
& \frac{1}{\left\|\vec{x}-\vec{x}^{\prime}\right\|}=\operatorname{sgn}\left(x_{1}^{\prime}\right) \oint_{|z|=1} \frac{d z}{2 \pi i} \oint_{\left|z^{\prime}\right|=1} \frac{d z^{\prime}}{2 \pi i} \\
& \quad \widetilde{G}\left(z,-\left[x_{2}-i x_{3}\right]+2 x_{1} z+\left[x_{2}+i x_{3}\right] z^{2} ; z^{\prime},-\left[x_{2}^{\prime}-i x_{3}^{\prime}\right]+2 x_{1}^{\prime} z^{\prime}+\left[x_{2}^{\prime}+i x_{3}^{\prime}\right] z^{\prime 2}\right)
\end{aligned}
$$

holds, unless all of the inequalities

$$
\left|x_{1}^{\prime}\right| \leq\|\vec{x}\| \leq\left\|\vec{x}^{\prime}\right\| \text { and } 4 x_{1}^{2} \leq x_{2}^{2}+x_{3}^{2} \text { and } 4 x_{1}^{\prime 2} \leq x_{2}^{\prime 2}+x_{3}^{\prime 2}
$$

are satisfied. If condition (2.20) holds, there are poles along the integration path which need a special treatment. Identity (2.19) does not necessarily hold for values of $\vec{x}$ and $\vec{x}^{\prime}$ that do not satisfy (2.20). This is because when we derive (2.18) from (2.2) $-(2.3)$ and (2.17), we have to change the order of integration and summation, and for an infinite series that does not necessarily converge.

If, on the other hand, $\|\vec{x}\|>\left\|\vec{x}^{\prime}\right\|$, then (2.19) still holds, except that $\operatorname{sgn}\left(x_{1}^{\prime}\right)$ needs to be replaced by $\operatorname{sgn}\left(x_{1}\right)$. The analog of the condition (2.20) is now

$$
\left|x_{1}\right| \leq\left\|\vec{x}^{\prime}\right\| \leq\|\vec{x}\| \text { and } 4 x_{1}^{2} \leq x_{2}^{2}+x_{3}^{2} \text { and } 4 x_{1}^{\prime 2} \leq x_{2}^{\prime 2}+x_{3}^{\prime 2}
$$

The mini-twistor transform $G\left(\mathfrak{t}, \mathfrak{t}^{\prime}\right)$ is not uniquely defined, because we can, for example, add an arbitrary meromorphic function that has no poles in the region $|z| \leq 1$, and we can also add an arbitrary meromorphic function with no poles in the region $|z| \geq 1$ (including $z=\infty$ ). We can also add functions with similar properties for $z^{\prime}$. Note also that the propagator (2.18) is not invariant under translations. The total translation generators can be read off from (2.10). When acting on holomorphic functions, they reduce to

$$
P_{+}=-i \frac{\partial}{\partial w}-i \frac{\partial}{\partial w^{\prime}}, \quad P_{1}=i z \frac{\partial}{\partial w}+i z^{\prime} \frac{\partial}{\partial w^{\prime}}, \quad P_{-}=i z^{2} \frac{\partial}{\partial w}+i z^{\prime 2} \frac{\partial}{\partial w^{\prime}} .
$$

It can be checked that $P_{ \pm} \widetilde{G}$ and $P_{1} \widetilde{G}$ do not vanish.

It is interesting that the off-shell propagator can be recast in terms of mini-twistors, in some region of parameter space. This seems to be the $D=3$ analog of the off-shell twistor propagator of [16]. It is possible that these formulas could be used to convert Feynman diagram rules, which are usually expressed in terms of momenta or coordinates, to diagrams in terms of mini-twistor variables. We were unsuccessful in putting such rules to practical use. This is partly because (2.19) only holds under the assumption (2.20), and partly because individual Feynman diagrams of gauge theories are not gauge invariant. 


\subsection{Minkowski space $\mathbb{R}^{2,1}$}

We can also define mini-twistor space for Minkowski signature. Pick a Majorana representation of the Clifford algebra, say,

$$
\Gamma^{0}=\left(\begin{array}{cc}
0 & 1 \\
-1 & 0
\end{array}\right), \quad \Gamma^{1}=\left(\begin{array}{cc}
-1 & 0 \\
0 & 1
\end{array}\right), \quad \Gamma^{2}=\left(\begin{array}{ll}
0 & 1 \\
1 & 0
\end{array}\right) .
$$

For a 3 -momentum $\vec{k}=\left(k^{0}, k^{1}, k^{2}\right)$, set

$$
k_{\mu} \Gamma^{\mu}=\left(\begin{array}{cc}
-k_{1} & k_{2}+k_{0} \\
k_{2}-k_{0} & k_{1}
\end{array}\right)=\left(\begin{array}{cc}
-k^{1} & k^{2}-k^{0} \\
k^{2}+k^{0} & k^{1}
\end{array}\right) \Longrightarrow k_{\alpha}{ }^{\beta}=k_{\mu}\left(\Gamma^{\mu}\right)_{\alpha}{ }^{\beta} .
$$

For a lightlike $\vec{k}$ we get

$$
0=\operatorname{det}\left(k_{\mu} \Gamma^{\mu}\right) \Longrightarrow k_{\alpha}{ }^{\beta}=\lambda_{\alpha} \tilde{\lambda}^{\beta}
$$

and if $\vec{k}$ is real, $\lambda$ and $\tilde{\lambda}$ are also real.

We get mini-twistor space by Fourier transforming with respect to $\tilde{\lambda}$ :

$$
e^{i \vec{k} \cdot \vec{x}} \longrightarrow \frac{1}{(2 \pi)^{2}} \int d^{2} \tilde{\lambda} e^{i x^{\alpha}{ }_{\beta} \lambda_{\alpha} \tilde{\lambda}^{\beta}} e^{i \tilde{\lambda}^{\beta} \mu_{\beta}}=\delta^{(2)}\left(x^{\alpha}{ }_{\beta} \lambda_{\alpha}+\mu_{\beta}\right),
$$

where

$$
x_{\beta}^{\alpha}=\left(\begin{array}{cc}
-x^{1} & x^{2}+x^{0} \\
x^{2}-x^{0} & x^{1}
\end{array}\right) .
$$

The condition

$$
\mu_{\beta}+x_{\beta}^{\alpha} \lambda_{\alpha}=0
$$

is related to the incidence relation (2.5) as follows. Expanding the components we get

$$
-\mu_{1}=x^{1}{ }_{1} \lambda_{1}+x^{2}{ }_{1} \lambda_{2}=-x^{1} \lambda_{1}+\left(x^{2}-x^{0}\right) \lambda_{2}, \quad-\mu_{2}=x^{1}{ }_{2} \lambda_{1}+x^{2}{ }_{2} \lambda_{2}=\left(x^{2}+x^{0}\right) \lambda_{1}+x^{1} \lambda_{2} \text {. }
$$

Now set

$$
z=\frac{\lambda_{1}}{\lambda_{2}}, \quad w=\frac{\mu_{1} \lambda_{2}-\mu_{2} \lambda_{1}}{\left(\lambda_{2}\right)^{2}}
$$

Then we find from (2.23) that

$$
w=2 z x^{1}-\left(x^{2}-x^{0}\right)+\left(x^{2}+x^{0}\right) z^{2} .
$$

Renaming $x^{0} \rightarrow i x^{3}$, the Minkowski incidence relation (2.25) becomes the Euclidean version (2.5). For Minkowski space, $z$ and $w$ are real. $z$ takes values in $\mathbb{R P}^{1} \simeq S^{1}$ and $w$ takes values in its tangent space, so together they parameterize the tangent bundle $T S^{1} \simeq \mathbb{R} \times S^{1}$. 


\section{Dimensional reduction}

We can calculate amplitudes of $D=3$ super Yang-Mills theory by dimensional reduction of $D=4$ amplitudes. For this purpose, we need to understand the connection [10 between the twistor space $\mathbb{C P}^{3} \backslash \mathbb{C P}^{1}$ of $\mathbb{R}^{4}$ and the mini-twistor space $T \mathbb{C P}^{1}$ of $\mathbb{R}^{3}$. We will review this connection in 3.2 . after a brief review of dimensional reduction for super Yang-Mills theory. For a recent comprehensive review of various aspects of $D=3$ Yang-Mills theory see [17.

\subsection{Field theory}

Euclidean $D=4$ Yang-Mills theory with $N=4$ supersymmetry has an $S U(4)$ R-symmetry group and $S U(2)_{L} \times S U(2)_{R}$ is the (double cover of) the rotation group. We will now review the $S U(2)_{L} \times S U(2)_{R} \times S U(4)$ representations of the fields, and at the same time introduce our notation. The field content is given by: a bosonic gauge field $A_{i}(i=1, \ldots, 4)$ in $(\mathbf{2}, \mathbf{2}, \mathbf{1})$, bosonic scalars $\Phi^{I}(I=1, \ldots, 6)$ in $(\mathbf{1}, \mathbf{1}, \mathbf{6})$, fermionic left-spinors $\psi_{\alpha}^{A}(A=1, \ldots, 4$ and $\alpha=1,2)$ in $(\mathbf{2}, \mathbf{1}, \mathbf{4})$, and fermionic right-spinors $\psi_{\dot{\alpha} A}(\dot{\alpha}=\dot{1}, \dot{2})$ in $(\mathbf{1}, \mathbf{2}, \overline{\mathbf{4}})$.

Dimensional reduction to $D=3$ proceeds by taking all the fields to be independent of $x_{4}$ and defining $\Phi^{7}:=A_{4}$. The (double cover of the) rotation group in (Euclidean) $D=3$ is $S U(2)$ and the distinction between dotted and undotted spinors disappears. The $D=3 \mathrm{R}$-symmetry group is $\operatorname{Spin}(7)$. We will now review the $S U(2) \times \operatorname{Spin}(7)$ representations of the $D=3$ fields. The fermions $\psi_{\alpha}^{A}$ and $\psi_{\dot{\alpha} A}$ combine to form fields in the $(\mathbf{2}, \mathbf{8})$, the gauge field components $A_{1}, \ldots, A_{3}$ form a gauge field in the $(\mathbf{3}, \mathbf{1})$ [that is dual to a scalar in $D=3$ ], and the scalars $\Phi^{I}(I=1, \ldots, 7)$ are in the $(\mathbf{1}, \mathbf{7})$ representation.

We will denote the $D=3$ fermions by $\chi_{\alpha}^{a}$ with $a=1, \ldots, 8$ being the index of the spinor representation of $s o(7)$ and $\alpha=1,2$ the $D=3$ spacetime spinor index. The $D=3$ Lagrangian is given by

$$
\begin{aligned}
g_{3}^{2} \mathcal{L}=\operatorname{tr}\left(\frac{1}{4} F_{i j} F^{i j}+\right. & \frac{1}{2} \sum_{i=1}^{7} D_{i} \Phi^{I} D^{i} \Phi^{I}-\frac{1}{4} \sum_{I, J}\left[\Phi^{I}, \Phi^{J}\right]^{2} \\
& \left.+\sum_{a=1}^{8} \chi_{\alpha}^{a} \sigma^{i \alpha \beta} \partial_{i} \chi_{\beta}^{a}+\sum_{a, b, I} \epsilon^{\alpha \beta} \Gamma_{a b}^{I} \Phi^{I} \chi_{\alpha}^{a} \chi_{\beta}^{b}\right)
\end{aligned}
$$

where $g_{3}$ is the $D=3$ coupling constant, $\epsilon^{\alpha \beta}$ is the standard antisymmetric lowering and raising matrix for 2-component spinors, $\sigma^{i \alpha \beta}$ are Pauli matrices, and $\Gamma_{a b}^{I}$ are $s o(7)$ Dirac matrices. 
Helicity in $D=3$ is defined as follows. We Wick rotate to Minkowski metric $\mathbb{R}^{2,1}$ and let $k^{\mu}(\mu=0, \ldots, 2)$ be a 3 -momentum of a massless particle. Choose a reference frame and let $\vec{k} \equiv k \vec{n} \in \mathbb{R}^{2}$ be the spatial component of $k^{\mu}$, with $\vec{n}$ a unit vector. Then, in the temporal gauge $A_{0}=0$, a $( \pm)$ helicity photon has a wave-function $\vec{A}$ (the two-component spatial part of $A_{i}$ ) satisfying $\vec{n} \times \vec{A}= \pm i \Phi^{7}$. This can be described more conveniently as follows. In $D=3$ a photon is equivalent to a scalar $\Phi^{8}$, by duality. The field-strength is then given by

$$
F_{i j}=\epsilon_{i j l} \partial_{l} \Phi^{8}
$$

A $( \pm)$ helicity photon then satisfies $\Phi^{7}= \pm i \Phi^{8}$. Note that the condition for a particular helicity breaks the $\operatorname{Spin}(7)$ R-symmetry to $\operatorname{Spin}(6) \simeq S U(4)$. We will see later in 3.3 that, indeed, our super-mini-twistor space only has a manifest $S U(4)$ symmetry and not $\operatorname{Spin}(7)$.

\subsection{Dimensional reduction of $D=4$ twistor space}

What is the relation between the twistor space $\mathbb{C P}^{3} \backslash \mathbb{C P}^{1}$ of $\mathbb{C}^{4}$ (regarded here as complexified $\mathbb{R}^{2,2}$ ) and the mini-twistor space $T \mathbb{C P}^{1}$ of $\mathbb{R}^{3}$ ? The answer is that $\mathbb{C P}^{3} \backslash \mathbb{C P}^{1}$ is a fibration over $T \mathbb{C P}^{1}\left[10\right.$. To see this, consider an arbitrary lightlike 4 -momentum $k^{\mu}$ in $\mathbb{C}^{4}$. It can be written as

$$
k_{\alpha \dot{\alpha}}=\lambda_{\alpha} \tilde{\lambda}_{\dot{\alpha}}
$$

In accordance with the Clifford algebra in (2.22), we choose the Majorana-Weyl spinor representation of $\mathbb{R}^{2,2}$ as

$$
\sigma_{\alpha \dot{\alpha}}^{0}=\left(\begin{array}{cc}
0 & -1 \\
1 & 0
\end{array}\right), \quad \sigma_{\alpha \dot{\alpha}}^{1}=\left(\begin{array}{cc}
1 & 0 \\
0 & -1
\end{array}\right), \quad \sigma_{\alpha \dot{\alpha}}^{2}=\left(\begin{array}{cc}
0 & -1 \\
-1 & 0
\end{array}\right), \quad \sigma_{\alpha \dot{\alpha}}^{3}=\left(\begin{array}{cc}
-1 & 0 \\
0 & -1
\end{array}\right),
$$

where we are working in signature $(+--+)$. Now suppose that we set $k^{3}=0$, so that $k^{\mu}$ will lie in $\mathbb{C}^{3}$ (complexified $\mathbb{R}^{2,1}$ ). This implies the constraint

$$
k^{\alpha \dot{\alpha}} \sigma_{\alpha \dot{\alpha}}^{3}=0 \Longrightarrow \lambda^{1} \tilde{\lambda}^{\dot{1}}+\lambda^{2} \tilde{\lambda}^{\dot{2}}=0
$$

In general, we will refer to the direction on which we dimensionally reduce as the $4^{\text {th }}$ direction. We can now see how a choice of direction for dimensional reduction naturally defines a fibration structure of $\mathbb{C P}^{3} \backslash \mathbb{C P}^{1}$. For example, the choice of $4^{\text {th }}$ direction in (3.3) defines the fibration by the condition that two twistors $(\lambda, \mu)$ and $\left(\lambda^{\prime}, \mu^{\prime}\right)$ in $\mathbb{C P}^{3} \backslash \mathbb{C P}^{1}$ belong to the same fiber if

$$
\lambda_{\alpha}^{\prime}=\lambda_{\alpha}, \quad \mu_{\dot{1}}^{\prime}=\mu_{\dot{1}}-t \lambda^{1}, \quad \mu_{\dot{2}}^{\prime}=\mu_{\dot{2}}-t \lambda^{2}
$$


for some $t \in \mathbb{C}$. The equivalence relation (3.4) arises naturally from (3.3) if we recall that the twistor transform of $\mathbb{R}^{2,2}$ is the Fourier transform from $\tilde{\lambda}$ to $\mu$. In general, had we chosen another direction $n^{\mu}$ on which to dimensionally reduce [instead of a unit vector $(0,0,0,1)$ ], we would have gotten the condition

$$
\lambda_{\alpha}^{\prime}=\lambda_{\alpha}, \quad \mu_{\dot{\alpha}}^{\prime}=\mu_{\dot{\alpha}}-t n_{\mu} \sigma_{\alpha \dot{\alpha}}^{\mu} \lambda^{\alpha}
$$

To see that (3.4) gives $T \mathbb{C P}^{1}$ as the base of the fibration, consider the two patches of $\mathbb{C P}^{3} \backslash \mathbb{C P}^{1}$, defined by the conditions $\lambda_{1} \neq 0$ and $\lambda_{2} \neq 0$, respectively. If $\lambda_{2} \neq 0$ we can set $z=\lambda_{1} / \lambda_{2}$, and rescale by $\lambda_{2}$ to get

$$
\left(\lambda_{1}, \lambda_{2}, \mu^{\dot{1}}, \mu^{\dot{2}}\right) \rightarrow\left(z, 1, \mu^{\dot{1}} / \lambda_{2}, \mu^{\dot{2}} / \lambda_{2}\right) .
$$

After raising and lowering indices, the equivalence relation (3.4) can be written as

$$
\lambda_{\alpha}^{\prime}=\lambda_{\alpha}, \quad \mu^{\prime \dot{1}}=\mu^{\dot{1}}+t \lambda_{1}, \quad \mu^{\prime \dot{2}}=\mu^{\dot{2}}+t \lambda_{2} .
$$

Therefore, it has a unique representative given by $\mu^{\dot{2}}=0$. We get to that representative by picking $t=-\mu^{\dot{2}} / \lambda_{2}$ in (3.5) , and using $\mu^{\prime}$ instead of $\mu$. Thus, the twistor

$$
\left(z, 1, \frac{\mu^{\dot{1}}}{\lambda_{2}}+t z, \frac{\mu^{\dot{2}}}{\lambda_{2}}+t\right)=\left(z, 1, \frac{\mu^{\dot{1}} \lambda_{2}-\lambda_{1} \mu^{\dot{2}}}{\left(\lambda_{2}\right)^{2}}, 0\right)
$$

represents the equivalence class (3.4). We set

$$
w:=\frac{\mu^{\dot{1}} \lambda_{2}-\lambda_{1} \mu^{\dot{2}}}{\left(\lambda_{2}\right)^{2}} .
$$

Now consider the other patch $\lambda_{1} \neq 0$. By similar arguments

$$
\left(1, \frac{1}{z}, 0, \frac{\mu^{\dot{2}} \lambda_{1}-\lambda_{2} \mu^{\dot{1}}}{\left(\lambda_{1}\right)^{2}}\right)
$$

represents (3.4). In this patch we choose the coordinates

$$
z^{\prime}=\frac{\lambda_{2}}{\lambda_{1}}=\frac{1}{z}, \quad w^{\prime}=\frac{\mu^{\dot{2}} \lambda_{1}-\lambda_{2} \mu^{\dot{1}}}{\left(\lambda_{1}\right)^{2}}=-\frac{w}{z^{2}} .
$$

A given spacetime point $x \in \mathbb{R}^{2,2}$ corresponds to a holomorphic section on $\mathbb{T}\left(\mathbb{R}^{2,2}\right)$ through the incidence relation [4]:

$$
\mu_{\dot{\alpha}}+x_{\alpha \dot{\alpha}} \lambda^{\alpha}=0
$$

In particular, if $x \in \mathbb{R}^{2,1}\left(x^{0^{\prime}}=0\right)$, the incidence relation gives

$$
\mu^{\dot{1}}=x^{1 \dot{1}} \lambda_{1}+x^{2 \dot{1}} \lambda_{2}=x^{1} \lambda_{1}-\left(x^{2}-x^{0}\right) \lambda_{2}, \quad \mu^{\dot{2}}=x^{1 \dot{2}} \lambda_{1}+x^{2 \dot{2}} \lambda_{2}=-\left(x^{2}+x^{0}\right) \lambda_{1}-x^{1} \lambda_{2},
$$


where we used the conventions (3.2). This together with (3.6) leads to the same three-dimensional incidence relation as (2.25), except that $z$ and $w$ are now complex numbers. Again, (2.25) becomes (2.5) by taking $x^{0} \rightarrow i x^{3}$. Therefore, $(z, w)$ and $\left(z^{\prime}, w^{\prime}\right)$ parameterize the mini-twistor space $T \mathbb{C P}^{1}$ of $\mathbb{R}^{3}$ exactly as described in $\$ 2.2$

Thus, the twistor space $\mathbb{C P}^{3} \backslash \mathbb{C P}^{1}$ of $\mathbb{C}^{4}$ is a fibration over the mini-twistor space $T \mathbb{C P}^{1}$ of $\mathbb{R}^{3}$. The fiber is $F \simeq \mathbb{C}$ and the structure group is the group $\mathbb{C}$ of translations of $\mathbb{C}$. To see this, note that on the patch of $T \mathbb{C P}^{1}$ where $z$ is a good coordinate, $u:=\mu^{2} / \lambda_{2}$ is a good coordinate on $F$, and on the other patch where $z^{\prime}$ is a good coordinate, $u^{\prime}:=\mu^{1} / \lambda_{1}$ is a good coordinate on $F$. On the intersection of the two patches, where both $z$ and $z^{\prime}$ are good coordinates,

$$
u^{\prime}=\frac{\mu^{\dot{1}}}{\lambda_{1}}=u+\frac{w}{z} .
$$

We will see in 93.4 that tree-level $D=3$ amplitudes in mini-twistor space $T \mathbb{C P}^{1}$ can be obtained from tree-level $D=4$ amplitudes in twistor space $\mathbb{C P}^{3} \backslash \mathbb{C P}^{1}$ by integration over the fiber $F$.

To understand the fibration structure more geometrically, note that $\mu=0$ defines a rational curve (which is homeomorphic to $\mathbb{C P}^{1}$ ) in $\mathbb{C P}^{3} \backslash \mathbb{C P}^{1}$. The normal bundle to the curve $\mu=0$ is isomorphic to the direct sum $\mathcal{O}(1) \oplus \mathcal{O}(1)$ of line-bundles. [Embedded in $\mathbb{C P}^{3} \backslash \mathbb{C P}^{1}$, the normal bundle to $\mu=0$ can be parameterized as $\left(\lambda_{1}, \lambda_{2}, d \mu^{\dot{1}}, d \mu^{\dot{2}}\right) \sim\left(z, 1, \xi_{1}, \xi_{2}\right)$ for $z=\lambda_{1} / \lambda_{2} \neq \infty$ and $\left(\lambda_{1}, \lambda_{2}, d \mu^{\dot{1}}, d \mu^{\dot{2}}\right) \sim\left(1, z^{\prime}, \xi_{1}^{\prime}, \xi_{2}^{\prime}\right) \equiv\left(1,1 / z, \xi_{1} / z, \xi_{2} / z\right)$ for $z=1 / z^{\prime} \neq 0$. Thus, the normal bundle is $\mathcal{O}(1) \oplus \mathcal{O}(1)$. See also [19]-22] for a related discussion and further details.] This holomorphic vector bundle has nowhere-vanishing holomorphic sections. For example, we define a section $\mathfrak{s}$ of $\mathcal{O}(1) \oplus \mathcal{O}(1)$ as follows. Set $\mathfrak{s}(z, 1)=(z, 1, z, 1)$ for $z \neq \infty$ and $\mathfrak{s}\left(1, z^{\prime}\right)=\left(1, z^{\prime}, 1, z^{\prime}\right)$ for $z^{\prime}=1 / z \neq \infty$. Obviously, the section values agree on the intersection of the two patches because $(z, 1, z, 1) \sim\left(\lambda_{1}, \lambda_{2}, \lambda_{1}, \lambda_{2}\right) \sim\left(1, z^{\prime}, 1, z^{\prime}\right)$, and $\mathfrak{s}$ is nowhere zero.

Based on the section $\mathfrak{s}$, we can define the sub-bundle of $\mathcal{O}(1) \oplus \mathcal{O}(1)$ as $c \mathfrak{s}(c \in \mathbb{C})$, which is a trivial line-bundle over $\mu=0$, since $c$ is a good global coordinate for the line fibers. Modding out $\mathcal{O}(1) \oplus \mathcal{O}(1)$ by this trivial line bundle, we get a quotient space. This means that we impose the equivalence relation on $\mathcal{O}(1) \oplus \mathcal{O}(1)$ by $c \mathfrak{s}$ :

$$
\left(\xi_{1}, \xi_{2}\right)+c(z, 1) \sim\left(\xi_{1}, \xi_{2}\right), z \neq \infty ; \quad\left(\xi_{1}^{\prime}, \xi_{2}^{\prime}\right)+c^{\prime}\left(1, z^{\prime}\right) \sim\left(\xi_{1}^{\prime}, \xi_{2}^{\prime}\right), z^{\prime} \neq \infty
$$

We get the representatives $(\eta, 0)=\left(\xi_{1}-\xi_{2} z, 0\right)$ and $\left(0, \eta^{\prime}\right)=\left(0, \xi_{2}^{\prime}-\xi_{1}^{\prime} z^{\prime}\right)$ by choosing $c=-\xi_{2}$ and $c^{\prime}=-\xi_{1}^{\prime}$. It can be easily shown that $\eta^{\prime}=-\eta / z^{2}$ and therefore the quotient space is a line bundle $\mathcal{O}(2)$. 
The resulting $\mathcal{O}(2)$ line bundle can be identified with the mini-twistor space $T \mathbb{C P}^{1}$. The $\mu=0$ rational curve is then identified with the base of $T \mathbb{C P}^{1}$. We denote the projection by

$$
\pi: \mathbb{C P}^{3} \backslash \mathbb{C P}^{1} \rightarrow T \mathbb{C P}^{1}
$$

These observations can be readily modified to spacetime $\mathbb{R}^{2,2}$ with signature $(++--)$, for which the twistor space is $\mathbb{R P}^{3} \backslash \mathbb{R P}^{1}$. All the equations above still apply, but $z, w$ and $\lambda, \mu$ should be real. In particular, if we choose a timelike $4^{\text {th }}$ direction $\left(0^{\prime}\right)$ for dimensional reduction we get $D=3$ Minkowski space $\mathbb{R}^{2,1}$. The choice of $4^{\text {th }}$ direction defines a fibration structure on twistor space $\mathbb{R P}^{3} \backslash \mathbb{R} \mathbb{P}^{1}$, with the projection

$$
\pi^{\prime}: \mathbb{R P}^{3} \backslash \mathbb{R} \mathbb{P}^{1} \rightarrow T \mathbb{R} \mathbb{P}^{1}
$$

The base of this fibration is the mini-twistor space of $D=3$ Minkowski space $T \mathbb{R P}^{1}$. The incidence relation is (2.25), as discussed in $\$ 2.7$

\subsection{Dimensional reduction of $D=4$ super-twistor space}

The dimensional reduction of supertwistor space $\mathbb{C P}^{3 \mid 4}$ proceeds in a similar fashion. We take homogeneous variables

$$
\left(\lambda_{1}, \lambda_{2}, \mu^{\dot{1}}, \mu^{\dot{2}}, \theta^{1}, \theta^{2}, \theta^{3}, \theta^{4}\right)
$$

on $\mathbb{C P}^{3 \mid 4}$ and begin with the patch $\lambda_{2} \neq 0$. At the end of $\$ 3.2$ we identified the three-dimensional mini-twistor space $T \mathbb{C P}^{1}$ with a quotient of the normal bundle to the $\mu=0$ rational curve, where we modded out by a trivial sub-bundle. We can repeat the same procedure for $\mathbb{C P}^{3 \mid 4}$. Pick the rational curve given by $\mu=0$ and $\theta=0$. The normal superspace is a sum of $\mathcal{O}(1) \oplus \mathcal{O}(1)$ corresponding to the $\mu$ directions, and four copies of anti-commuting $\mathcal{O}(1)$ spaces, corresponding to the $\theta$ directions. As before, a choice of $4^{\text {th }}$ direction on which to dimensionally reduce defines, as in (3.4), a trivial sub-bundle of the commuting $\mathcal{O}(1) \oplus \mathcal{O}(1)$ vector bundle. Modding out by this subspace leaves

$$
\mathcal{O}(2 \mid 0) \oplus \mathcal{O}(0 \mid 1)^{4}
$$

where the first factor is commuting and the last four are anticommuting. This is the $D=3$ super-mini-twistor space. It can be covered with two patches $U_{1}$ and $U_{2}$. On the first patch $U_{1}$ the local coordinates are $\left(z, w, \theta_{1}, \ldots, \theta_{4}\right)$ (where $z, w$ are commuting), and on the second patch 
$U_{2}$ the local coordinates are $\left(z^{\prime}, w^{\prime}, \theta_{1}^{\prime}, \ldots, \theta_{4}^{\prime}\right)$, with transition functions

$$
z^{\prime}=\frac{1}{z}, \quad w^{\prime}=-\frac{w}{z^{2}}, \quad \theta_{i}^{\prime}=\frac{1}{z} \theta_{i} \quad(i=1 \ldots 4)
$$

generalizing (2.6) . Note that this super-mini-twistor space is a noncompact Calabi-Yau supermanifold [18]. Defining $x$ to be the generator of the cohomology group $H^{2}\left(\mathbb{C P}^{1}, \mathbb{Z}\right)$, we find that the first Chern class of $\mathbb{C P}^{1}$ is $2 x$, the $\mathcal{O}(2 \mid 0)$ factor contributes another $2 x$, and the four $\mathcal{O}(0 \mid 1)$ anti-commuting factors contribute $-x$ each, to a total of 0 .

\subsection{Tree-level amplitudes}

For simplicity, we will start with $D=4$ with signature $\mathbb{R}^{2,2}$ and dimensionally reduce by picking a timelike $4^{\text {th }}$ direction to obtain $D=3$ Minkowski space $\mathbb{R}^{2,1}$. We have seen in $\$ 3.2$ that the twistor space $\mathbb{R} \mathbb{P}^{3} \backslash \mathbb{R} \mathbb{P}^{1}$ is a fibration over $T \mathbb{R P}^{1}$. We will now argue that the tree-level $D=3$ amplitudes are obtained by integrating the $D=4$ tree-level amplitudes over the fiber.

In [4, the twistor space $\mathbb{R} \mathbb{P}^{3} \backslash \mathbb{R} \mathbb{P}^{1}$ is obtained as the parameter space of the Fourier transforms of functions of $k_{\alpha \dot{\alpha}}^{\mu}=\lambda_{\alpha} \tilde{\lambda}_{\dot{\alpha}}$ with respect to $\tilde{\lambda}$. Alternatively, if we have a function $\widetilde{F}(\lambda, \mu)$ on the twistor space, we can get back a function $F(\lambda, \tilde{\lambda})$ by Fourier transforming with respect to $\mu$.

To dimensionally reduce a given amplitude of the $D=4$ theory in its twistor space $\mathbb{R P}^{3} \backslash \mathbb{R P}^{1}$, we need to enforce the condition (3.3.3). Let us integrate $\widetilde{F}(\lambda, \mu)$ over the fiber of the fibration (3.9). We get

$$
\begin{aligned}
\frac{1}{2 \pi} \int d t \widetilde{F}\left(\lambda_{1}, \lambda_{2}, \mu_{\dot{1}}+t \lambda^{1}, \mu_{\dot{2}}+t \lambda^{2}\right) & =\frac{1}{2 \pi} \int d t d^{2} \tilde{\lambda} e^{i \tilde{\lambda}^{\mathrm{i}}\left(\mu_{\mathrm{i}}+t \lambda^{1}\right)+i \tilde{\lambda}^{\dot{2}}\left(\mu_{\dot{2}}+t \lambda^{2}\right)} F\left(\lambda_{1}, \lambda_{2}, \tilde{\lambda}^{\mathrm{i}}, \tilde{\lambda}^{\dot{2}}\right) \\
& =\int d^{2} \tilde{\lambda} e^{i \tilde{\lambda}^{\dot{\alpha}} \mu_{\dot{\alpha}}} \delta\left(\lambda^{1} \tilde{\lambda}^{\mathrm{i}}+\lambda^{2} \tilde{\lambda}^{\dot{2}}\right) F\left(\lambda_{1}, \lambda_{2}, \tilde{\lambda}^{\mathrm{i}}, \tilde{\lambda}^{\dot{2}}\right)
\end{aligned}
$$

Thus, integrating over the fiber is equivalent to inserting a delta function $\delta\left(\lambda^{1} \tilde{\lambda}^{i}+\lambda^{2} \tilde{\lambda}^{\dot{2}}\right)=\delta\left(k^{3}\right)$.

To transform an $n$-particle $D=4$ tree-level amplitude $A_{4}\left(\lambda^{(1)}, \mu^{(1)} ; \cdots ; \lambda^{(n)}, \mu^{(n)}\right)$ on $\mathbb{R} \mathbb{P}^{3} \backslash \mathbb{R P}^{1}$ to $T \mathbb{R P}^{1}$, all we need then is to integrate $A_{4}$ over the fibers of $n$ twistors:

$$
A_{3}\left(\lambda^{(1)}, \mu^{(1)} ; \cdots ; \lambda^{(n)}, \mu^{(n)}\right)=\int \prod_{j=1}^{n} d t_{j} A_{4}\left(\lambda^{(1)}, \mu^{(1)}+t_{1} \lambda^{(1)} ; \cdots ; \lambda^{(n)}, \mu^{(n)}+t_{n} \lambda^{(n)}\right) .
$$

In momentum space, each $d t_{j}$ integration inserts a $\delta\left(k_{j}^{3}\right)$, as in (3.11). Because of total 4momentum conservation, this is one $\delta\left(k_{j}^{3}\right)$ too many,

$$
\delta\left(\sum_{j=1}^{n} k_{j}^{3}\right) \prod_{j=1}^{n} \delta\left(k_{j}^{3}\right)=\delta(0) \prod_{j=1}^{n} \delta\left(k_{j}^{3}\right) .
$$


The infinite factor $\delta(0)$ can be regularized if we take a compact $4^{\text {th }}$ dimension. The singular $\delta(0)$ can then be interpreted as the spatial size $(\sim 2 \pi R)$ in the $4^{\text {th }}$ direction, which is absorbed in the $D=3$ coupling constant $g_{3}=g_{4} / 2 \pi R$. (Here $g_{4}$ is the $D=4$ coupling constant.) The resulting amplitude $A_{3}$ is invariant under the equivalence relation (3.4) for each particle separately. It can therefore be written as a function of only $\mathfrak{t}_{j}:=\pi^{\prime}\left(\lambda^{(j)}, \mu^{(j)}\right)$ (for $j=1 \ldots n$ ) [where the projection $\pi^{\prime}$ was defined in (3.9) $]$.

We are now ready to show that tree-level amplitudes have support on algebraic curves, as in $D=4$ [4]. Let $A_{3}\left(\mathfrak{t}_{1}, \ldots, \mathfrak{t}_{n}\right)$ be a tree-level $n$-point $D=3$ amplitude as a function of $n$ minitwistors in $T R \mathbb{P}^{1}$. As argued above, this amplitude can be obtained from a $D=4$ amplitude $A_{4}\left(\tilde{\mathfrak{t}}_{1}, \ldots, \tilde{\mathfrak{t}}_{n}\right)$ where $\tilde{\mathfrak{t}}_{i}$ is a twistor in $\mathbb{R} \mathbb{P}^{3} \backslash \mathbb{R} \mathbb{P}^{1}$ that projects to $\mathfrak{t}_{i}$ in the fibration above. That is, $A_{3}\left(\mathfrak{t}_{1}, \ldots, \mathfrak{t}_{n}\right)$ is obtained from $A_{4}\left(\tilde{\mathfrak{t}}_{1}, \ldots, \tilde{\mathfrak{t}}_{n}\right)$ by integrating with respect to $\tilde{\mathfrak{t}}_{1}, \ldots, \tilde{\mathfrak{t}}_{n}$ over the fibers above $\mathfrak{t}_{1}, \ldots, \mathfrak{t}_{n}$. In the notation of (3.9) we have $\pi^{\prime}\left(\tilde{\mathfrak{t}}_{i}\right)=\mathfrak{t}_{i}$. According to [4], $A_{4}$ is nonzero only if its arguments $\tilde{\mathfrak{t}}_{1}, \ldots, \tilde{\mathfrak{t}}_{n}$ lie on an algebraic curve of degree $d=q-1$, where $q$ is the number of negative helicity gluons. Since this curve is given by algebraic equations in the homogeneous coordinates of $\mathbb{R P}^{3}$, it can be analytically continued to $\mathbb{C P}^{3}$. Let this analytically continued curve be $\widetilde{\Sigma} \subset \mathbb{C P}^{3}$. According to [4, this curve must be of genus 0 , otherwise the amplitude vanishes. We denote its projection by $\Sigma=\pi(\widetilde{\Sigma}) \subset T \mathbb{C P}^{1}$, where we used $\pi$ from (3.8). The genus of a projection of a sphere cannot be $>0$, so $\Sigma$ is also of genus 0 . In the local coordinates $(z, w)$ on $T \mathbb{C P}^{1}, \Sigma$ can be expressed as a polynomial equation

$$
0=\sum_{r, s} C_{r, s} z^{r} w^{s}
$$

with some coefficients $C_{r, s} \in \mathbb{C}$. In order to reduce Witten's conjectures 4 ] to $D=3$, we need to define the degree of $\Sigma$. We can do that by identifying $T \mathbb{C P}^{1}$ with an open subset of weighted projective space $W \mathbb{C P}^{1,1,2}$ as follows. Let $\xi_{1}, \xi_{2}, \xi_{3}$ be projective coordinates on $W \mathbb{C P}^{1,1,2}$ defined with the equivalence relation

$$
\left(\xi_{1}, \xi_{2}, \xi_{3}\right) \sim\left(\zeta \xi_{1}, \zeta \xi_{2}, \zeta^{2} \xi_{3}\right), \quad 0 \neq \zeta \in \mathbb{C}
$$

If we take the singular point $\xi_{1}=\xi_{2}=0$ out of $W \mathbb{C P}^{1,1,2}$, we get the mini-twistor space $T \mathbb{C P}^{1}$ as follows,

$$
z=\frac{\xi_{2}}{\xi_{1}}, \quad w=\frac{\xi_{3}}{\xi_{1}^{2}} .
$$


The equation (3.13) for $\Sigma$ becomes

$$
0=\sum_{r, s} C_{r, s} \xi_{1}^{-r-2 s} \xi_{2}^{r} \xi_{3}^{s}
$$

We define the degree of $\Sigma$ by

$$
\tilde{d}(\Sigma):=\max _{r, s}(r+2 s) .
$$

Multiplying (3.15) by $\xi_{1}^{\tilde{d}(\Sigma)}$ we see that $\Sigma$ can be represented by a homogeneous polynomial of degree $\tilde{d}(\Sigma)$ in weighted projective space $W \mathbb{C P}^{1,1,2}$.

We will see below that the dimensional reduction of a $D=4$ twistor amplitude that corresponds to twistors lying on a holomorphic curve of degree $d$ in $\mathbb{C P}^{3}$ reduces to a mini-twistor amplitude with the mini-twistors restricted to lie on a curve of the form (3.13) and of degree $\tilde{d}(\Sigma)=2 d$. Thus, it follows immediately from the observations in [4] that the $D=3$ tree-level mini-twistor amplitude with $q$ negative helicity gluons and $n-q$ positive helicity gluons is nonzero only if the $n$ mini-twistors lie on a genus 0 curve $\Sigma$ of degree $\tilde{d}(\Sigma)=2(q-1)$ in $T \mathbb{C P}^{1}$. The Minkowski tree-level amplitude is nonzero if the $n$ mini-twistors lie on an algebraic curve in $T \mathbb{R} \mathbb{P}^{1} \simeq \mathbb{R}^{1} \times S^{1}$ with the same degree and genus as above.

Suppose that the $D=4$ tree-level amplitude is supported on a "complete intersection," which is defined as the zero set of two homogeneous polynomials $f_{1}\left(\lambda_{\alpha}, \mu^{\dot{\alpha}}\right)$ and $f_{2}\left(\lambda_{\alpha}, \mu^{\dot{\alpha}}\right)$, of degrees $d_{1}$ and $d_{2}$, respectively. Then the degree of such a curve is $d=d_{1} d_{2}$. The $D=4$ amplitude can be schematically written as

$$
A_{4}\left(\tilde{\mathfrak{t}}_{1}, \ldots, \tilde{\mathfrak{t}}_{n}\right)=\int_{\mathcal{M}}\left[d f_{1}\right]\left[d f_{2}\right] \prod_{i=1}^{n} \delta\left(f_{1}\left(\lambda_{\alpha}^{(i)}, \mu^{(i) \dot{\alpha}}\right)\right) \delta\left(f_{2}\left(\lambda_{\alpha}^{(i)}, \mu^{(i) \dot{\alpha}}\right)\right) \mathcal{A}\left(\lambda_{\alpha}^{(i)}, \mu^{(i) \dot{\alpha}}\right),
$$

where the integration is performed over the moduli space $\mathcal{M}$ of genus 0 , degree $d$ curves in $\mathbb{C P}^{3}$ of this particular "complete intersection" form (or equivalently, over the space of polynomials $f_{1}\left(\lambda_{\alpha}, \mu^{\dot{\alpha}}\right)$ and $f_{2}\left(\lambda_{\alpha}, \mu^{\dot{\alpha}}\right)$ with specified degrees, after some appropriate identifications). According to our previous discussion, the $D=3$ amplitude can then be obtained by integrating the $D=4$ amplitude over the fibers of the twistors $\tilde{\mathfrak{t}}_{i}$ :

$A_{3}\left(\mathfrak{t}_{1}, \ldots, \mathfrak{t}_{n}\right)=\int_{\mathcal{M}}\left[d f_{1}\right]\left[d f_{2}\right] \int \prod_{i=1}^{n} d t_{i} \delta\left(f_{1}\left(\lambda_{\alpha}^{(i)}, \mu^{(i) \dot{\alpha}}+t_{i} \lambda_{\alpha}^{(i)}\right)\right) \delta\left(f_{2}\left(\lambda_{\alpha}^{(i)}, \mu^{(i) \dot{\alpha}}+t_{i} \lambda_{\alpha}^{(i)}\right)\right) \mathcal{A}\left(\lambda_{\alpha}^{(i)}, \mu^{(i) \dot{\alpha}}+t_{i} \lambda_{\alpha}^{(i)}\right)$.

Therefore, our $D=3$ amplitude will not vanish provided there exist two polynomials $f_{1}\left(\lambda_{\alpha}, \mu^{\dot{\alpha}}\right)$ and $f_{2}\left(\lambda_{\alpha}, \mu^{\dot{\alpha}}\right)$ and some value $t_{i}$ for each $i$ so that both $f_{1}\left(\lambda_{\alpha}^{(i)}, \mu^{(i) \dot{\alpha}}+t_{i} \lambda_{\alpha}^{(i)}\right)$ and $f_{2}\left(\lambda_{\alpha}^{(i)}, \mu^{(i) \dot{\alpha}}+\right.$ $\left.t_{i} \lambda_{\alpha}^{(i)}\right)$ are non-zero for each $i$. 
Now, for two fixed polynomials $f_{1}\left(\lambda_{\alpha}, \mu^{\dot{\alpha}}\right)$ and $f_{2}\left(\lambda_{\alpha}, \mu^{\dot{\alpha}}\right)$, what is the condition for such values $t_{i}$ to exist? To answer this question, let us expand the two polynomials $f_{1}\left(\lambda_{\alpha}^{(i)}, \mu^{(i) \dot{\alpha}}+t_{i} \lambda_{\alpha}^{(i)}\right)$ and $f_{2}\left(\lambda_{\alpha}^{(i)}, \mu^{(i) \dot{\alpha}}+t_{i} \lambda_{\alpha}^{(i)}\right)$ and group the terms in decreasing order in $t_{i}$. As these polynomials are homogeneous of degrees $d_{1}$ and $d_{2}$, we will get two polynomial equations,

$$
\begin{gathered}
a_{0} t_{i}^{d_{1}}+a_{1} t_{i}^{d_{1}-1}+\cdots+a_{d_{1}}=0, \\
b_{0} t_{i}^{d_{2}}+b_{1} t_{i}^{d_{2}-1}+\cdots+b_{d_{2}}=0,
\end{gathered}
$$

where the coefficients $a_{0}, \ldots, a_{d_{1}}$ and $b_{0}, \ldots, b_{d_{2}}$ are of degrees $d_{1}$ and $d_{2}$, respectively, in $\lambda_{\alpha}^{(i)}$ and $\mu^{(i) \dot{\alpha}}$. But the condition for the existence of a simultaneous solution $t_{i}$ for the above two polynomial equations is that the resultant of the polynomials be zero. By explicitly writing down the resultant, one can easily check that each term of the resultant is of degree $d_{2}$ in $a_{0}, \ldots, a_{d_{1}}$ and of degree $d_{1}$ in $b_{0}, \ldots, b_{d_{2}}$. Hence the condition for the $D=3$ amplitude to be non-zero is that the twistors $\tilde{\mathfrak{t}}_{i}=\left(\lambda^{(i)}, \mu^{(i)}\right)$ satisfy a certain polynomial equation of degree

$$
\tilde{d}=d_{1} d_{2}+d_{2} d_{1}=2 d_{1} d_{2}=2 d .
$$

As $z$ is linear and $w$ is quadratic in $\lambda_{\alpha}$ and $\mu^{\dot{\alpha}}$ after setting $\lambda_{2}=1$ in (3.6), the degree of the resulting polynomial when expressed in terms of $z$ and $w$ becomes $\tilde{d}=2 d$, according to our definition in (3.16).

We will end this subsection with an analysis of dimensional reduction for Euclidean signature. We have seen at the end of 2.2 that a harmonic scalar function on $\mathbb{R}^{3}$ is mini-twistor transformed to an element of the sheaf cohomology $H^{1}\left(T \mathbb{C P}^{1}, \widetilde{\Omega}^{1}\right)$, where $\widetilde{\Omega}$ was the pullback of the sheaf $\mathcal{O}(-2)$ over $\mathbb{C P}^{1}$. By the arguments of [4], for each external particle the scattering amplitude must be an element of the dual space, which in our case also happens to be $H^{1}\left(T \mathbb{C P}^{1}, \widetilde{\Omega}^{1}\right)$. As explained in $\S 2.5$ of $[4$, for each external particle of helicity $h$ the $D=4$ amplitude is an element of the sheaf cohomology $H^{1}\left(\mathbb{C P}^{3} \backslash \mathbb{C P}^{1}, \mathcal{O}(h-2)\right)$. We have seen in 3.2 that $\mathbb{C P}^{3} \backslash \mathbb{C P}^{1}$ is a fibration over $T \mathbb{C P}^{1}$ with $\mathbb{C}$ fibers.

Given a $D=3$ harmonic function, we can lift it to a $D=4$ harmonic function that is invariant under translations in the $4^{\text {th }}$ direction. What is the corresponding statement for the twistor transforms? The pull-back of an element of $H^{1}\left(T \mathbb{C P}^{1}, \widetilde{\Omega}^{1}\right)$, with respect to the projection (3.8), is an element of $H^{1}\left(\mathbb{C P}^{3} \backslash \mathbb{C P}^{1}, \mathcal{O}(-2)\right)$ that is invariant under translations along the fiber.

\subsection{Explicit examples of amplitudes}

We will now give a few examples of dimensionally reduced tree-level amplitudes. 
The tree-level maximally helicity-violating (MHV) twistor amplitude with 2 gluons of negative helicity and $(n-2)$ gluons of positive helicity is given by formula (3.3) of [4],

$$
A\left(\lambda^{(i)}, \mu^{(i)}\right)=i g_{4}^{n-2} \int d^{4} x \prod_{i=1}^{n} \delta^{2}\left(\mu_{\dot{\alpha}}^{(i)}+x_{\alpha \dot{\alpha}} \lambda^{(i) \alpha}\right) \frac{\left\langle\lambda^{(r)}, \lambda^{(s)}\right\rangle^{4}}{\prod_{i=1}^{n}\left\langle\lambda^{(i)}, \lambda^{(i+1)}\right\rangle},
$$

where we use the standard notation

$$
\left\langle\lambda^{(i)}, \lambda^{(j)}\right\rangle:=\lambda_{\alpha}^{(i)} \lambda^{(j) \alpha}, \quad \lambda^{(n+1)}:=\lambda^{(1)}
$$

Here, the $r$-th and $s$-th gluons are of negative helicity. To get the $D=3$ twistor amplitude, we have to replace the $D=4$ coupling constant $g_{4}$ by the $D=3$ coupling constant $g_{3}$, replace $d^{4} x$ by $d^{3} x$, and integrate over the fibers:

$$
i g_{3}^{n-2} \int d^{3} x \prod_{i=1}^{n} d t_{i} \delta\left(\mu_{\dot{1}}^{(i)}+t \lambda^{(i) 1}+x_{\alpha \dot{1}} \lambda^{(i) \alpha}\right) \delta\left(\mu_{\dot{2}}^{(i)}+t \lambda^{(i) 2}+x_{\alpha \dot{2}} \lambda^{(i) \alpha}\right) \frac{\left\langle\lambda^{(r)}, \lambda^{(s)}\right\rangle^{4}}{\prod_{i=1}^{n}\left\langle\lambda^{(i)}, \lambda^{(i+1)}\right\rangle} .
$$

Note that if we first perform the fiber integrations over $d t_{i}$ and leave the $d^{3} x$ integral for last, the integrand will be independent of the $4^{\text {th }}$ component of $x$. This is because momentum conservation in the $4^{\text {th }}$ direction is already enforced, since the $d t_{i}$ integrations make sure that the $4^{\text {th }}$ component of momentum is zero.

We calculate

$$
\int d t \delta\left(\mu_{\dot{1}}+t \lambda^{1}+x_{\alpha \dot{1}} \lambda^{\alpha}\right) \delta\left(\mu_{\dot{2}}+t \lambda^{2}+x_{\alpha \dot{2}} \lambda^{\alpha}\right)=\frac{1}{\left(\lambda_{2}\right)^{2}} \delta\left(w+\left[x^{2}-i x^{3}\right]-2 x^{1} z-\left[x^{2}+i x^{3}\right] z^{2}\right),
$$

where we used (3.6) and $z=\lambda_{1} / \lambda_{2}$. The argument of the $\delta$-function enforces the incidence relation (2.5), which is a polynomial of degree 2 . Note also that

$$
\left\langle\lambda^{(i)}, \lambda^{(i+1)}\right\rangle=\left(z_{i+1}-z_{i}\right)\left(\lambda_{2}^{(i)} \lambda_{2}^{(i+1)}\right)
$$

The $D=3 \mathrm{MHV}$ amplitude is therefore

$$
i g_{3}^{n-2} \int d^{3} x \prod_{i=1}^{n} \delta\left(w_{i}+\left[x^{2}-i x^{3}\right]-2 x^{1} z_{i}-\left[x^{2}+i x^{3}\right] z_{i}^{2}\right) \frac{\left(z_{r}-z_{s}\right)^{4}}{\prod_{i=1}^{n}\left(z_{i+1}-z_{i}\right)} .
$$

Here, we used the fact that on the patch where $\lambda_{2} \neq 0$ we can scale the factor $\lambda_{2}$ to 1 . If, instead, we had included all the $\lambda_{2}$ factors, we would have needed an extra factor of $\left(\lambda_{2}^{(r)}\right)^{4}\left(\lambda_{2}^{(s)}\right)^{4} / \prod_{i=1}^{n}\left(\lambda_{2}^{(i)}\right)^{4}$. This factor indicates that the MHV amplitude in $D=3$ is homogeneous of degree -4 and 4 
for each negative and positive helicity particle, respectively. On the patch $\lambda_{1} \neq 0$ we define the twistor amplitude as

$$
A_{3}\left(w_{i}^{\prime}, z_{i}^{\prime}\right)=i g_{3}^{n-2} \int d^{3} x \prod_{i=1}^{n} \delta\left(w_{i}^{\prime}+\left[x_{2}-i x_{3}\right]-2 x_{1} z_{i}^{\prime}-\left[x_{2}+i x_{3}\right] z_{i}^{\prime 2}\right) \frac{\left(z_{r}^{\prime}-z_{s}^{\prime}\right)^{4}}{\prod_{i=1}^{n}\left(z_{i+1}^{\prime}-z_{i}^{\prime}\right)} .
$$

The $\delta$-functions enforce the incidence relations. In the geometrical language of 92.3 , the amplitude is nonzero only if all $n$ mini-twistors, which are oriented lines in $\mathbb{R}^{3}$, intersect at a common point.

Our next example is the "googly" description of the tree-level amplitudes with helicities ---++ . It was shown in [4] that these are supported on genus zero, degree two curves in the $D=4$ twistor space $\mathbb{R P}^{3}$. It was also shown there that this condition is equivalent to saying that the amplitude is nonzero only if (i) the five points $\tilde{\mathfrak{t}}_{i}=\left(\lambda^{(i)}, \mu^{(i)}\right)$ lie on a common $\mathbb{R P}^{2} \subset \mathbb{R P}^{3}$, and (ii) the five points lie on a common conic section contained in that $\mathbb{R P}^{2}$. But once the first condition is satisfied, the second one is automatic, because a generic set of five points in $\mathbb{R} \mathbb{P}^{2}$ is contained in a unique conic section. Therefore we can schematically write the $D=4$ amplitude $\mathrm{as}^{3}$

$$
A_{4}\left(\tilde{\mathfrak{t}}_{1}, \ldots, \tilde{\mathfrak{t}}_{5}\right)=\int_{\mathcal{M}}[d a][d b] \prod_{i=1}^{5} \delta\left(\sum_{I=1}^{4} a_{I} Z_{i}^{I}\right) \delta\left(\sum_{I, J=1}^{4} b_{I J} Z_{i}^{I} Z_{i}^{J}\right) \mathcal{A}\left(Z_{1}^{I}, \ldots, Z_{5}^{I}\right)
$$

where

$$
\left(Z_{i}^{1}, Z_{i}^{2}, Z_{i}^{3}, Z_{i}^{4}\right)=\left(\lambda_{\alpha}^{(i)}, \mu^{(i) \dot{\alpha}}\right)
$$

are the coordinates of the five twistors $\tilde{\mathfrak{t}}_{i}$, and the integration is to be performed over the moduli space of genus zero, degree two algebraic curves in $\mathbb{R P}^{3}$. (This space is parameterized by the coefficients $a_{I}$ and $b_{I J}$, with appropriate identification.)

As before, dimensional reduction in twistor space is achieved by replacing $\mu^{(i) \dot{\alpha}}$ with $\mu^{(i) \dot{\alpha}}+$ $t_{i} \lambda_{\alpha}^{(i)}$ and integrating over $t_{i}$ for each $i$. So in the $D=3$ amplitude, the first delta function for each $i$ in the above expression becomes

$$
\delta\left(a_{1} \lambda_{1}^{(i)}+a_{2} \lambda_{2}^{(i)}+a_{3}\left(\mu^{(i) \dot{1}}+t_{i} \lambda_{1}^{(i)}\right)+a_{4}\left(\mu^{(i) \dot{2}}+t_{i} \lambda_{2}^{(i)}\right)\right)
$$

Integration over $t_{i}$ then amounts to solving the equation

$$
a_{1} \lambda_{1}^{(i)}+a_{2} \lambda_{2}^{(i)}+a_{3}\left(\mu^{(i) \dot{1}}+t_{i} \lambda_{1}^{(i)}\right)+a_{4}\left(\mu^{(i) \dot{2}}+t_{i} \lambda_{2}^{(i)}\right)=0
$$

\footnotetext{
${ }^{3} \mathrm{~A}$ precise formula for the googly amplitude was derived from B-model calculation in [23]. For our purposes, this schematic form is enough.
} 
for $t_{i}$ and plugging it into the $t_{i}$ in the argument of the second delta function. Therefore, we end up with a product of delta functions whose arguments look like

$$
b_{11}\left(\lambda_{1}^{(i)}\right)^{2}+b_{12} \lambda_{1}^{(i)} \lambda_{2}^{(i)}+b_{13} \lambda_{1}^{(i)}\left(\mu^{(i) \dot{1}}+t_{i} \lambda_{1}^{(i)}\right)+b_{14} \lambda_{1}^{(i)}\left(\mu^{(i) \dot{2}}+t_{i} \lambda_{2}^{(i)}\right)+\cdots+b_{44}\left(\mu^{(i) \dot{2}}+t_{i} \lambda_{2}^{(i)}\right)^{2},
$$

where $t_{i}$ are to be replaced with the solution of the above equation. After clearing the denominators, these arguments become homogeneous polynomials of degree four in $\lambda_{\alpha}^{(i)}$ and $\mu^{(i) \dot{\alpha}}$. Then from (3.6), we see that when expressed in terms of $z_{i}$ and $w_{i}$, they will become a polynomial of degree four in $T \mathbb{R} P^{1}$ according to our definition of degree in (3.16). We conclude that dimensionally reduced tree-level ---++ amplitudes are nonvanishing only if the five twistors $\mathfrak{t}_{i}$ lie on a common curve of degree four in $D=3$ twistor space. This result agrees with our claim in $\$ 3.4$

But this condition is actually trivial, because a generic set of five points in $T \mathbb{R} \mathbb{P}^{1}$ always lies on a common curve of degree four. To see this, it suffices to simply write down the most general form of degree four curves in $T \mathbb{R} \mathbb{P}^{1}$ :

$$
f(z, w)=w^{2}+c_{1} z^{2} w+c_{2} z^{4}+c_{3} z^{3}+c_{4} z w+c_{5} z^{2}+c_{6} w+c_{7} z+c_{8}=0
$$

We have eight parameters $c_{1}, \ldots, c_{8}$ at our disposal but have to satisfy only five constraints $f\left(z_{i}, w_{i}\right)=0$, so generically such curves exist.

In summary, we have seen that (a) for MHV amplitudes, the $D=3$ twistor amplitudes after dimensional reduction are nonvanishing only if the twistors lie on a common algebraic curve with genus 0 and degree 2 in $T \mathbb{R P}^{1}$, while (b) for the googly description of ---++ amplitudes, there does not exist such nontrivial criterion. The difference between these two cases is easy to understand in geometrical terms. In the MHV case, the $D=4$ amplitude in (3.18) contains two $\delta$-functions for each $i$. We can think of the first set of $\delta$-functions as enforcing the $n$ points $\left(\lambda^{(i)}, \mu^{(i)}+t_{i} \lambda^{(i)}\right)$ to lie on a common $\mathbb{R P}^{2}$. Then the second set of $\delta$-functions further demands that the $n$ points lie on a common line contained in that $\mathbb{R P}^{2}$. We can always pick $t_{i}$ for each $i$ so that the $n$ points lie on a common $\mathbb{R P}^{2}$, but after fixing $t_{i}$, these points will in general not lie on a common line if $n>2$. Therefore, we still have a nontrivial criterion for nonvanishing amplitudes after dimensional reduction.

In contrast, for ---++ amplitudes, the first set of $\delta$-functions in (3.20) requires that the five twistor points lie on a common $\mathbb{R P}^{2}$. Again, this can always be achieved by a judicious choice of $t_{i}$. But then the second set of $\delta$-functions demands that the five points lie on a common 
conic section, which is satisfied automatically. So there is no nontrivial criterion for nonvanishing amplitudes.

\subsection{Physical interpretation of the holomorphic curves}

In this subsection we will present a geometrical and physical interpretation of the holomorphic curves in mini-twistor space. There is an interesting connection between holomorphic curves in mini-twistor space $T \mathbb{C P}^{1}$ and (real) surfaces of minimal area in the physical space $\mathbb{R}^{3}$. A minimal area surface is constructed from a holomorphic curve $\Sigma \subset T \mathbb{C P}^{1}$ as follows [10]. Given a point $\left(z_{0}, w_{0}\right)$ on $\Sigma$, we can write the equation for $\Sigma$ near $\left(z_{0}, w_{0}\right)$ as

$w=w_{0}+a_{1}\left(z-z_{0}\right)+a_{2}\left(z-z_{0}\right)^{2}+O\left(z-z_{0}\right)^{3}=\left(w_{0}-a_{1} z_{0}+a_{2} z_{0}^{2}\right)+\left(a_{1}-2 a_{2} z_{0}\right) z+a_{2} z^{2}+O\left(z-z_{0}\right)^{3}$.

Dropping the $O\left(z-z_{0}\right)^{3}$ terms, we can approximate the curve by a quadratic equation and find a vector $\vec{x} \in \mathbb{C}^{3}$ such that this quadratic equation will look like the incidence relation (2.5). Given the coefficients of $1, z, z^{2}$ in (3.21) , we therefore define $\vec{x} \equiv\left(x_{1}, x_{2}, x_{3}\right) \in \mathbb{C}^{3}$ as the unique solution to the linear equations

$$
-\left(x_{2}-i x_{3}\right)=\left(w_{0}-a_{1} z_{0}+a_{2} z_{0}^{2}\right), \quad 2 x_{1}=\left(a_{1}-2 a_{2} z_{0}\right), \quad x_{2}+i x_{3}=a_{2}
$$

Thus, each point on $\Sigma$ defines a point $\vec{x} \in \mathbb{C}^{3}$ and the collection of these points defines a holomorphic curve in $\mathbb{C}^{3}$. The projection of this curve to $\mathbb{R}^{3}$, i.e., the collection of points $\operatorname{Re} \vec{x} \equiv$ $\left(\operatorname{Re} x_{1}, \operatorname{Re} x_{2}, \operatorname{Re} x_{3}\right)$, is a minimal area (real) surface in $\mathbb{R}^{3}$. Furthermore, there is a one-to-one map from minimal area surfaces in $\mathbb{R}^{3}$ to holomorphic curves in $T \mathbb{C P}^{1}$. For more details, we refer the reader to the appendix of [10].

We saw in 33.4 that a physical amplitude defines a holomorphic curve in $T \mathbb{C P}^{1}$. From the above discussion it follows that a physical amplitude defines a minimal area surface in $\mathbb{R}^{3}$. What is its significance?

We will address the Minkowski variant of this question. We have seen in $\$ 2.7$ that the minitwistor space of $\mathbb{R}^{2,1}$ is $T \mathbb{R} \mathbb{P}^{1}$. For $\mathbb{R}^{2,1}$ the coordinates $z$ and $w$ are real, and so are the coefficients $a_{1}, a_{2}$, in (3.21). We define $\vec{x} \in \mathbb{R}^{3}$ by comparison with the Minkowski incidence relation (2.25),

$$
-\left(x_{2}-x_{0}\right)=w_{0}-a_{1} z_{0}+a_{2} z_{0}^{2}, \quad 2 x_{1}=a_{1}-2 a_{2} z_{0}, \quad x_{2}+x_{0}=a_{2}
$$

The solution to (3.23) defines a unique point in $\mathbb{R}^{2,1}$ for every point on the real curve $\Sigma \in T \mathbb{R P}^{1}$. 


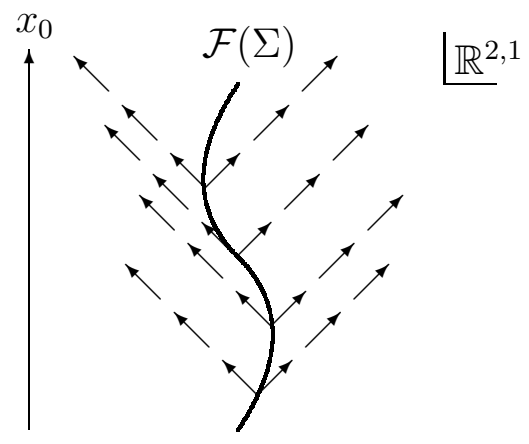

Figure 2: The outgoing waves of the scattering process can be described as a physical disturbance that is emanating from the "focal curve" $\mathcal{F}(\Sigma)$.

The collection of these points form a curve $\mathcal{F}(\Sigma)$ in $\mathbb{R}^{2,1}$. What is the physical significance of $\mathcal{F}(\Sigma)$ ?

Take a particular amplitude with $n$ mini-twistors $\mathfrak{t}_{1}, \ldots, \mathfrak{t}_{n}$, and let us consider a particular scattering experiment to which this amplitude would contribute. Thus, we pick $m<n$ twistors $\mathfrak{t}_{1}, \ldots, \mathfrak{t}_{m}$ to describe incoming particles, and assume that $\mathfrak{t}_{m+1}, \ldots, \mathfrak{t}_{n}$ describe outgoing particles. We also assume that the number of negative helicities $q$ is fixed and that $m$ and $\mathfrak{t}_{1}, \ldots, \mathfrak{t}_{m}$ are chosen so that there is a unique holomorphic curve, of the corresponding degree $2(q-1)$, that passes through all the mini-twistors $\mathfrak{t}_{1}, \ldots, \mathfrak{t}_{m}$. The amplitude will then be nonzero only if $\mathfrak{t}_{m+1}, \ldots, \mathfrak{t}_{n}$ lie on that curve.

A mini-twistor $\mathfrak{t}=(z, w)$ describes an incoming planar shockwave of the form

$$
\phi_{\mathfrak{t}}(\vec{x}) \sim \delta\left(w-2 z x^{1}+\left[x^{2}-x^{0}\right]-\left[x^{2}+x^{0}\right] z^{2}\right)
$$

that travels at the speed of light. The scattering process is therefore a collision of $m$ incoming shockwaves. What comes out?

For fixed mini-twistors $\mathfrak{t}_{m+2}, \ldots, \mathfrak{t}_{n}$, the outgoing wave-function of the $(m+1)^{s t}$ particle is a linear combination of shock-waves of the form (3.24), and in general all mini-twistors $\mathfrak{t}$ that lie on $\Sigma$ can contribute. Suppose a particular $\mathfrak{t} \equiv \mathfrak{t}_{m+1} \equiv\left(z_{0}, w_{0}\right)$ contributes to the outgoing wave-function. Then nearby mini-twistors $\mathfrak{t}+\delta \mathfrak{t} \equiv\left(z_{0}+\delta z, w_{0}+\delta w\right)$ will also contribute provided that they lie on $\Sigma$. But near $\left(z_{0}, w_{0}\right)$ the curve $\Sigma$ looks like the parabola (3.21). This implies that up to second order in $\delta \mathfrak{t}$, all shock-waves $\phi_{\mathfrak{t}+\delta \mathfrak{t}}$ are nonzero at the point $\vec{x}$ defined by (3.23). Thus, the outgoing wave-function of the $(m+1)^{s t}$ particle is a linear combination of shockwaves, all of which pass through the "focal curve" $\mathcal{F}(\Sigma)$. The outgoing wave-function is therefore a disturbance emanating from $\mathcal{F}(\Sigma)$. (See Figure 2, ) 
As an example, take the curve $\Sigma$ given by

$$
w=2 z^{3} .
$$

Then from (3.21) and (3.23) we get the parametric equation for $\mathcal{F}(\Sigma)$ in the form

$$
\vec{x} \equiv\left(x_{0}, x_{1}, x_{2}\right)=\left(z^{3}+3 z,-3 z^{2}, 3 z-z^{3}\right) .
$$

Note that the tangent to this curve is null in $\mathbb{R}^{2,1}$. It is easy to see that this is true for a generic curve $\Sigma$. In the special case of MHV amplitudes, the focal curve $\mathcal{F}(\Sigma)$ degenerates to a point.

\subsection{Dimensional reduction in B-model twistor string theory}

In 4, Witten proposed a reformulation of $D=4$ super Yang-Mills theory as a B-model on supertwistor space $\mathbb{C P}^{3 \mid 4}$. An alternative approach was presented in [13] [24]. In this section we will make a few observations about the twistor string theory of $D=3$ super Yang-Mills theory.

The twistor string theory that describes $D=3$ super Yang-Mills with $N=8$ supersymmetry is the topological B-model on the $D=3$ supertwistor space from $\$ 2.5$ It can be obtained from the $D=4$ twistor space $\mathbb{C P}^{3 \mid 4}$ by dimensional reduction. For the B-model, dimensional reduction is implemented by gauging one generator of $S L(4 \mid 4)^{4}$ that corresponds to translations in the $4^{\text {th }}$ direction. (This is somewhat reminiscent of the construction of topological $\sigma$-models in [25].) Let

$$
Z_{1} \equiv \lambda_{1}, Z_{2} \equiv \lambda_{2}, Z_{3} \equiv \mu_{\dot{1}}, Z_{4} \equiv \mu_{\dot{2}}, \theta^{1}, \ldots, \theta^{4}
$$

be projective coordinates on $\mathbb{C P}^{3 / 4}$ with the equivalence relation

$$
\left(Z_{1}, \ldots, Z_{4}, \theta^{1}, \ldots, \theta^{4}\right) \sim\left(\zeta Z_{1}, \ldots, \zeta Z_{4}, \zeta \theta^{1}, \ldots, \zeta \theta^{4}\right) .
$$

We choose the basis so that the translation generator $P_{4}$ in the $4^{\text {th }}$ direction acts as

$$
P_{4}: \quad \delta Z_{1}=\delta Z_{2}=0, \quad \delta Z_{3}=\epsilon Z_{1}, \quad \delta Z_{4}=-\epsilon Z_{2}, \quad \delta \theta^{1}=\cdots=\delta \theta^{4}=0 .
$$

The transformation (3.27) is a symmetry of the B-model, since it preserves the complex structure of $\mathbb{C P}^{3 \mid 4}$ and the holomorphic measure.

The resulting topological B-model on the $D=3$ mini-twistor space can also be viewed as a limit of a discrete orbifold of the B-model on $\mathbb{C P}^{3 \mid 4}$. To construct this orbifold, pick a constant

\footnotetext{
${ }^{4}$ We are now considering the 4 -d space to have signature $(+--+)$ and will reduce along one of the time-like directions.
} 


\begin{tabular}{|cclc|}
\hline \hline field & Variable & Worldsheet & $S U(4)$ representation \\
\hline$\tilde{Z}, \tilde{W}, \bar{Z}, \bar{W}$ & commuting & scalars & \\
$\bar{\eta}^{z}, \bar{\eta}^{\bar{w}}$, & anti-commuting & scalars & $\mathbf{1}$ \\
$\vartheta_{z}, \vartheta_{w}$ & anti-commuting & scalars & $\mathbf{1}$ \\
$\rho^{z}, \rho^{w}$ & anti-commuting & 1-forms & $\mathbf{1}$ \\
$\Theta^{A}$ & anti-commuting & scalars & $\mathbf{4}$ \\
$\bar{\Theta}_{A}$ & anti-commuting & scalars & $\overline{\mathbf{4}}$ \\
$\bar{\eta}_{A}$ & commuting & scalars & $\overline{\mathbf{4}}$ \\
$\vartheta_{A}$ & commuting & scalars & $\overline{\mathbf{4}}$ \\
$\rho^{A}$ & commuting & 1-forms & $\mathbf{4}$ \\
\hline \hline
\end{tabular}

Table 1: The fields of the worldsheet B-model. Note that the index $z$ on some of the fields refers to the target space coordinate, and should not be confused with the worldsheet coordinate that is implicit.

parameter $r>0$ and define the group $\Gamma_{r} \simeq \mathbb{Z}$ generated by $\gamma_{r}=\exp \left(2 \pi i r P_{4}\right)$. It acts on $\mathbb{C P}^{3 \mid 4}$ as

$$
\begin{aligned}
\gamma_{r} \equiv e^{2 \pi i r P_{4}} \quad: \quad Z_{1} \mapsto Z_{1}, \quad Z_{2} \mapsto Z_{2}, \quad Z_{3} \mapsto Z_{3}+2 \pi r Z_{1} \\
Z_{4} \mapsto Z_{4}-2 \pi r Z_{2}, \quad \theta^{A} \mapsto \theta^{A} \quad(A=1, \ldots, 4)
\end{aligned}
$$

This map is compatible with the equivalence relation (3.26), and it also preserves the holomorphic superform $\epsilon^{I J K L} Z_{I} d Z_{J} \wedge d Z_{K} \wedge d Z_{L} \wedge d \theta^{1} \wedge \cdots \wedge d \theta^{4}$. The orbifold $\mathbb{C P}^{3 \mid 4} / \Gamma_{r}$ is therefore a CalabiYau superspace. The fixed-point set of $\Gamma_{r}$ is the subset $Z_{1}=Z_{2}=0$ which is the $\mathbb{C P}^{1 \mid 4}$ that is excised. The physical interpretation of the B-model on the $\Gamma_{r}$-orbifold is, of course, the twistor string worldsheet theory for $D=4$ super Yang-Mills theory compactified on a circle of radius $r$. In the limit $r \rightarrow 0$ we recover the $D=3$ mini-twistor space. The resulting worldsheet fields of the B-model on mini-twistor space are listed in Table 1. The BRST transformation laws are

$$
\begin{gathered}
\delta \overline{\tilde{Z}}=\bar{\eta}^{\bar{z}}, \quad \delta \overline{\tilde{W}}=\bar{\eta}^{\bar{w}}, \quad \delta \bar{\Theta}_{A}=\bar{\eta}_{A}, \\
\delta \rho^{z}=d \tilde{Z}, \quad \delta \rho^{w}=d \tilde{W}, \quad \delta \rho^{A}=d \Theta^{A} .
\end{gathered}
$$

The transformation laws of the remaining fields are zero.

\subsection{Dimensional reduction in Berkovits's twistor string theory}

Dimensional reduction can be performed similarly in Berkovits's model of the twistor string theory [13. In this model there are separate left- and right-moving worldsheet fields. The 
$S L(4 \mid 4)^{5}$ charged fields are $Z_{L}^{i}, Z_{R}^{i}, Y_{i L}, Y_{i R}(i=1, \ldots, 4)$ and $\Theta_{L}^{A}, \Theta_{R}^{A}, \Upsilon_{A L}, \Upsilon_{A R}(A=1, \ldots, 4)$, where $L(R)$ denotes a left-moving (right-moving) field. There is an additional $G L(1)$ gauge field $A$ under which $Z^{i}, \Theta^{A}$ have +1 charge and $Y_{i}, \Upsilon_{A}$ have -1 charge. Also, there are left-moving and right-moving chiral current algebras that give rise to the spacetime $S U(N)$ quantum numbers. The action is [13]

$$
S=\int d^{2} \mathfrak{z}\left[Y_{L i} \nabla_{\overline{\mathfrak{z}}} Z_{L}^{i}+\Upsilon_{L A} \nabla_{\overline{\mathfrak{z}}} \Theta_{L}^{A}+Y_{i R} \nabla_{\mathfrak{z}} Z_{R}^{i}+\Upsilon_{A R} \nabla_{\mathfrak{z}} \Theta_{R}^{A}\right]+S_{C},
$$

where $\nabla_{\mathfrak{z}}=\partial_{\mathfrak{z}}-A_{\mathfrak{z}}$ and $\nabla_{\overline{\mathfrak{z}}}=\partial_{\overline{\mathfrak{z}}}-A_{\overline{\mathfrak{z}}}$ are the covariant derivatives, and $S_{C}$ is the action of the chiral current algebras.

It is important to recall that even though (3.30) has cubic gauge interactions it is a conformally invariant theory. In fact, the equations of motion are

$$
\begin{array}{ccccc}
\nabla_{\overline{\mathfrak{z}}} Z_{L}^{i}=0, & \nabla_{\overline{\mathfrak{z}}} Y_{L i}=0, & \nabla_{\mathfrak{z}} Z_{R}^{i}=0, & \nabla_{\mathfrak{z}} Y_{R i}=0, & i=1, \ldots, 4, \\
\nabla_{\overline{\mathfrak{z}}} \Theta_{L}^{A}=0, & \nabla_{\overline{\mathfrak{z}}} \Upsilon_{L A}=0, & \nabla_{\mathfrak{z}} \Theta_{R}^{A}=0, & \nabla_{\mathfrak{z}} \Upsilon_{R A}=0, & A=1, \ldots, 4,
\end{array}
$$

and

$$
0=\sum_{i} Z_{L}^{i} Y_{L i}+\sum_{A} \Upsilon_{L A} \Theta_{L}^{A}, \quad 0=\sum_{i} Z_{R}^{i} Y_{R i}+\sum_{A} \Upsilon_{R A} \Theta_{R}^{A},
$$

and the gauge fields can be solved in terms of the other fields. For $Z_{L}^{1} \neq 0$ we can set

$$
A_{\overline{\mathfrak{z}}}=\partial_{\overline{\mathfrak{z}}} Z_{L}^{1} / Z_{L}^{1}
$$

Eliminating $A_{\overline{\mathfrak{z}}}$ from all the equations of motion (3.31), we find that all left-moving gauge invariant combinations (for example $Z_{L}^{2} / Z_{L}^{1}$ ) are holomorphic, and all right-moving gauge invariant combinations are anti-holomorphic. This holomorphicity condition, together with the analytic constraints (3.32), completely captures all the equations of motion. The theory is therefore conformally invariant, since the gauge invariant fields are holomorphic.

As was explained in 24], the path integral splits into sectors that are labeled by an "instanton number" $d$. This number represents the total $U(1) \subset G L(1)$ flux. Following [24], we gauge-fix the Weyl transformations on the worldsheet and the $G L(1)$ gauge field by setting the worldsheet metric in such a way that a field of $G L(1)$ charge $q$ and conformal dimension $h$ will be equivalent to a gauge neutral holomorphic field of conformal dimension $h+(d / 2) q$. This is a particularly convenient gauge fixing, because as explained in 24], all the left-moving fields are holomorphic

\footnotetext{
${ }^{5}$ We are still working in signature $(+--+)$.
} 
and all the right-moving field are anti-holomorphic. We can therefore use conformal field theory OPEs to calculate commutators.

(To avoid clutter, unless otherwise specified we will from now on write formulas only for the left-movers and suppress the $L$ subscripts.) Dimensional reduction proceeds in a similar fashion as for the B-model. Instead of (3.28), we have

$$
\begin{aligned}
\gamma_{r} \equiv e^{2 \pi i r P_{4}}: \quad Z^{1} \mapsto Z^{1}, \quad Z^{2} \mapsto Z^{2}, \quad Z^{3} \mapsto Z^{3}+2 \pi r Z^{1}, \quad Z^{4} \mapsto Z^{4}-2 \pi r Z^{2}, \\
Y_{1} \mapsto Y_{1}-2 \pi r Y_{3}, \quad Y_{2} \mapsto Y_{2}+2 \pi r Y_{4}, \quad Y_{3} \mapsto Y_{3}, \quad Y_{4} \mapsto Y_{4}, \\
\Theta^{A} \mapsto \Theta^{A}, \quad \Upsilon_{A} \mapsto \Upsilon_{A} \quad(A=1, \ldots, 4),
\end{aligned}
$$

In the limit $r \rightarrow 0$ we gauge a continuous symmetry. We can do this by introducing an extra auxiliary gauge field $\widetilde{B}_{\mathfrak{z}}, \widetilde{B}_{\overline{\mathfrak{z}}}$ and modifying the covariant derivatives of $Z^{3}, Z^{4}$ to

$$
\nabla_{\overline{\mathfrak{z}}} Z^{3}=\partial_{\overline{\mathfrak{z}}} Z^{3}-A_{\overline{\mathfrak{z}}} Z^{3}-\widetilde{B}_{\overline{\mathfrak{z}}} Z^{1}, \quad \nabla_{\overline{\mathfrak{z}}} Z^{4}=\partial_{\overline{\mathfrak{z}}} Z^{4}-A_{\overline{\mathfrak{z}}} Z^{4}+\widetilde{B}_{\overline{\mathfrak{z}}} Z^{2}
$$

and similarly for the right-moving fields. Inserting these covariant derivatives into the action (3.30) and integrating over $\widetilde{B}_{\overline{\mathfrak{z}}}$, we get the constraint

$$
Y_{3} Z^{1}-Y_{4} Z^{2}=0
$$

Out of $Z^{1}, \ldots, Z^{4}, \Theta^{1}, \ldots, \Theta^{4}$ we can make the following $\widetilde{B}$-gauge invariant combinations: $W:=Z^{3} Z^{2}+$ $Z^{4} Z^{1}$ with $G L(1)$ charge +2 , and $Z^{1}, Z^{2}, \Theta^{A}$ with $G L(1)$ charge +1 . The constraint (3.35) allows us to define

$$
U:=\frac{Y_{3}}{Z^{2}}=\frac{Y_{4}}{Z^{1}}
$$

This field $U$ has $G L(1)$ charge -2 . It is well-defined provided that either $Z^{1} \neq 0$ or $Z^{2} \neq 0$, which is indeed always the case. We also define

$$
\tilde{Y}_{1}=Y_{1}-U Z^{4}, \quad \tilde{Y}_{2}=Y_{2}-U Z^{3}
$$

The action (3.30) together with the constraint (3.35) becomes,

$$
S=\int d^{2} \mathfrak{z}\left[\widetilde{Y}_{1} \nabla_{\overline{\mathfrak{z}}} Z^{1}+\widetilde{Y}_{2} \nabla_{\overline{\mathfrak{z}}} Z^{2}+U \nabla_{\overline{\mathfrak{z}}} W+\Upsilon_{A} \nabla_{\overline{\mathfrak{z}}} \Theta^{A}\right]+(\text { right-movers })+S_{C},
$$

where $S_{C}$ is the action of the current algebra and $\nabla_{\overline{\mathfrak{z}}}:=\partial_{\overline{\mathfrak{z}}}-A_{\overline{\mathfrak{z}}}$ is the covariant $G L(1)$ derivative.

The resulting theory is easy to interpret if we recall that mini-twistor space is equivalent to $W \mathbb{C P}^{1,1,2}$ [see (3.14)]. The fields $Z^{1}, Z^{2}$ and $W$ correspond to standard projective coordinates 


\begin{tabular}{|cccc|}
\hline \hline field & statistics & worldsheet & $G L(1)$ charge \\
\hline$Z^{1}, Z^{2}$ & commuting & scalars & 1 \\
$W$ & commuting & scalar & 2 \\
$\widetilde{Y}_{1}, \widetilde{Y}_{2}$ & commuting & vectors & -1 \\
$U$ & commuting & vector & -2 \\
$\Theta^{A}$ & anti-commuting & scalars & 1 \\
$\Upsilon^{A}$ & anti-commuting & vectors & -1 \\
\hline \hline
\end{tabular}

Table 2: The left-moving worldsheet fields of mini-twistor string theory à la Berkovits.

on $W \mathbb{C P}^{1,1,2}$ with weights 1,1 and 2 , respectively. The $G L(1)$ charges of those fields correspond to these weights. In addition, we have four anti-commuting fields $\Theta^{A}(A=1, \ldots, 4)$ with $G L(1)$ charges 1 . Each of the fields above has a canonical conjugate field. $\widetilde{Y}_{1}, \widetilde{Y}_{2}$ are the conjugates of $Z^{1}, Z^{2}$, and $U$ is the conjugate of $W$. The conjugate of $\Theta^{A}$ is $\Upsilon_{A}$. In addition, there are also left and right moving current algebras. As Berkovits and Motl 24] explained, the worldsheet path integral splits into discrete sectors labeled by the flux $d$ of the $G L(1)$ gauge field. The left-moving fields are listed in Table 2, The right-moving fields have similar quantum numbers.

The generators of the Poincaré algebra (2.10), (2.13) can easily be expressed in terms of the fields $Z^{1}, Z^{2}, W$ and their conjugates $\widetilde{Y}_{1}, \widetilde{Y}_{2}, U$. The worldsheet currents that correspond to the translation generators $P_{+}, P_{-}$and $P_{1}$ are

$$
\mathcal{P}_{+}=-U\left(Z^{1}\right)^{2}, \quad \mathcal{P}_{1}=U Z^{1} Z^{2}, \quad \mathcal{P}_{-}=U\left(Z^{2}\right)^{2}
$$

The transformation properties can be determined from the OPEs

$$
\begin{aligned}
Z^{i}(\mathfrak{z}) \tilde{Y}_{j}(0) & \sim \frac{1}{\mathfrak{z}}, \quad W(\mathfrak{z}) U(0) \\
Z^{i}(\mathfrak{z}) U(0) \sim Z^{i}(\mathfrak{z}) Z^{j}(0) \sim \widetilde{Y}_{i}(\mathfrak{z}) \widetilde{Y}_{j}(0) \sim W(\mathfrak{z}) W(0) \sim U(\mathfrak{z}) U(0) & \sim \text { regular } .
\end{aligned}
$$

The supersymmetry generators (2.12) can also be expressed as currents. The left-moving currents corresponding to the SUSY generators are

$$
\mathcal{Q}_{A+}=Z^{1} \Upsilon_{A}, \quad \overline{\mathcal{Q}}_{+}^{A}=-i Z^{1} \Theta^{A} U, \quad \mathcal{Q}_{A-}=Z^{2} \Upsilon_{A}, \quad \overline{\mathcal{Q}}_{-}^{A}=i Z^{2} \Theta^{A} U
$$

\section{9 $\operatorname{Spin}(7)$ R-symmetry}

The R-symmetry group of our $D=3$ super Yang-Mills theory is $\operatorname{Spin}(7)$. It acts linearly on the 8 supersymmetry generators, which transform as the spinor representation 8 of Spin(7). However, 
only an $S U(4)$ subgroup is manifest in mini-twistor string theory. This is the subgroup that acts linearly on $\Theta^{A}$ and can be identified with the $D=4$ R-symmetry, before dimensional reduction. ${ }^{6}$

It is not a big surprise that full Spin(7) symmetry is not explicit. Some symmetries are obscure in twistor string theory. A nice example is parity, which is not at all manifest in the B-model [71], but was cleverly identified by Berkovits and Motl [24] in the open twistor string model. In this subsection we will identify the full Spin(7) R-symmetry generators in Berkovits's model.

Let us first write down the commutation relations for the $\operatorname{Spin}(7)$ R-symmetry generators. We take a basis for the $s o(7)$ Lie algebra that consists of $s u(4)$ generators, which we denote by $T_{B}^{A}$, and 6 additional generators, which we denote by $T^{A B}=-T^{B A}(A, B=1, \ldots, 4)$. The commutation relations are

$$
\begin{aligned}
& {\left[T^{A}{ }_{B}, T^{C}{ }_{D}\right]=\delta_{D}^{A} T_{B}^{C}-\delta_{B}^{C} T_{D}^{A}, \quad\left[T_{B}^{A}, T^{C D}\right]=\delta_{B}^{D} T^{A C}-\delta_{B}^{C} T^{A D},} \\
& {\left[T^{A B}, T^{C D}\right]=-\epsilon^{A B C E} T_{E}^{D}+\epsilon^{A B D E} T_{E}^{C}+\epsilon^{C D A E} T^{B}{ }_{E}-\epsilon^{C D B E} T^{A}{ }_{E} .}
\end{aligned}
$$

To see how the missing R-symmetry generators $T^{A B}$ operate, let us look at a particular example of a $\operatorname{Spin}(7)$ multiplet of fields. According to [14], $D=4$ twistor string theory contains, in addition to the super Yang-Mills theory, a sector that describes conformal supergravity. Our example of a $\operatorname{Spin}(7)$ multiplet of fields will comprise of the dimensional reduction of some of these $D=4$ conformal supergravity fields. We will take a multiplet of fields that transform in the irreducible representation 35 (anti-symmetric 3-tensors) of Spin(7). This irreducible representation decomposes under $S U(4) \subset \operatorname{Spin}(7)$ as

$$
35=15+10+\overline{\mathbf{1 0}}
$$

Thus, we can construct our multiplet by combining fields that transform in the three irreducible representations on the right hand side of (3.43).

The fields of $D=4$ conformal supergravity are listed in Table 1 in $\S 4.2$ of [14]. We can obtain our multiplet by combining three irreducible $S U(4)_{R}$ representations from that list. In the notation of [14], we pick the fields $E_{A B}=E_{B A}, \bar{E}^{A B}=\bar{E}^{B A}$ and $V_{\mu}{ }^{A}$. The first is a $D=4$ space-time scalar in the $\overline{\mathbf{1 0}}$ of $S U(4)_{R}$; the second is a $D=4$ space-time scalar in the $\mathbf{1 0}$ of $S U(4)_{R}$, and the last one is a $D=4$ space-time vector in the $\mathbf{1 5}$ adjoint representation of

\footnotetext{
${ }^{6}$ We are now working in signature $(-+++)$ again. Had we started instead in signature $(-++-)$ and dimensionally reduced along a timelike direction, we would have had to work with the noncompact R-symmetry group $\operatorname{Spin}(4,3)$, which extends the $D=4$ group $\operatorname{Spin}(3,3) \sim S L(4)$.
} 
$S U(4)_{R}$. After dimensional reduction to $D=3$, the scalars $E_{A B}, \bar{E}^{A B}$ and the $4^{\text {th }}$ component $V_{4}{ }_{B}{ }_{B}$ of $V_{\mu}$ form a $\operatorname{Spin}(7)$ multiplet in the irreducible representation 35.

Now let us see how $\operatorname{Spin}(7)$ acts on $E_{A B}, \bar{E}^{A B}$ and $V_{4}{ }_{B}{ }_{B}$. The action of the $S U(4) \subset \operatorname{Spin}(7)$ generators $T^{A}{ }_{B}$ is obvious. The $\operatorname{Spin}(7)$ generators $T^{A B}$ must also transform these states to each other. It is, however, difficult to identify the $T^{A B}$ generators in the B-model version of mini-twistor string theory. Part of the problem is that there are no perturbative B-model string vertex operators that correspond to the fields $E_{A B}$. The vertex operator for the dimensionally reduced $\bar{E}^{B A}$ and the vertex operator for $V_{4}{ }^{A}{ }_{B}$ were constructed in [14], and we will recall them below, in (4.13) and (4.10). But the vertex operators for $E_{A B}$ are absent in the perturbative B-model. To understand this, recall that the states of a perturbative B-model with target space $X$ correspond to the sheaf cohomology $H^{p}\left(X, \wedge^{q} T X\right)$ where $T X$ is the holomorphic tangent bundle. Nonperturbatively, it is conjectured 38. that the B-model is S-dual to the A-model and therefore also has states that correspond to Dolbeault cohomology $H^{p}\left(X, \Omega^{q} X\right)$ [where $\Omega^{q} X$ is the sheaf of holomorphic $(q, 0)$-forms]. The states $E_{A B}$ are analogous to the latter. (See also the discussion after equation (2.12) of [14.) For recent developments in the nonperturbative formulation of the topological string theory see [39] 40] 41].

\subsection{0 $\operatorname{Spin}(7)$ in Berkovits's model}

It is easier, however, to identify $T^{A B}$ in Berkovits's model. As usual in a two-dimensional conformal field theory, the symmetry generators correspond to holomorphic and anti-holomorphic currents. We will denote these currents by $\mathcal{J}^{A B}$. Let us first identify the worldsheet currents that correspond to the generators $T_{B}^{A}$. They are easily constructed from the $S U(4)$ transformation properties of $\Theta^{A}$ and $\Upsilon_{A}$, and we get

$$
\mathcal{J}^{A}{ }_{B}:=\Upsilon_{B} \Theta^{A}-\frac{1}{4} \delta_{B}^{A} \Upsilon_{C} \Theta^{C}
$$

We claim that the worldsheet currents that correspond to $T^{A B}$ can be expressed, formally, as

$$
\mathcal{J}^{A B}:=U \Theta^{A} \Theta^{B}+\frac{1}{2} U^{-1} \epsilon^{A B C D} \Upsilon_{C} \Upsilon_{D}
$$

We determined the generators (3.44) by looking for expressions with total conformal dimension 1, GL(1) charge zero, and the correct residues in their OPEs among themselves and with the Poincaré currents (3.39) and supersymmetry currents (3.41). These residues are determined by the known commutation relations between the R-symmetry and super-Poincaré generators. For 
example, using the residues of the simple poles in the OPEs of the currents $\mathcal{J}^{A B}$ and $\mathcal{J}^{A}{ }_{B}$, one can verify the commutation relations (3.42).

The operator $U^{-1}$ that appears in (3.44) has to be defined carefully. It is required to have the OPE

$$
U^{-1}(\mathfrak{z}) U(0)=1+O(\mathfrak{z})
$$

This operator $U^{-1}$ can be handled by bosonization as follows. The $(U, W)$ system is very similar to the superconformal ghosts $(\beta, \gamma)$ of superstring theory, except that the conformal dimensions are shifted. Thus, $(U, W)$ can be bosonized in much the same way as the superconformal ghosts. (See, e.g., $§ 10.4$ of [46]. Bosonization of the $\left(Y_{I}, Z^{I}\right)$ fields has also been discussed in [24] 47], and see also [4].) Let $\phi$ be a chiral boson, and let $(\xi, \eta)$ be anti-commuting ghosts, with OPEs

$$
\phi(\mathfrak{z}) \partial \phi(0) \sim \frac{1}{\mathfrak{z}}, \quad \eta(\mathfrak{z}) \xi(0) \sim \frac{1}{\mathfrak{z}}
$$

(See $\S 10.4$ of [46].) The bosonization formulas are then

$$
W \simeq-e^{-\phi} \partial \xi, \quad U \simeq e^{\phi} \eta
$$

We can then take

$$
U^{-1} \simeq e^{-\phi} \xi
$$

and this has all the properties required of the inverse of $U$.

We can now use the R-symmetry operators (3.44) to complete partial Spin(7) multiplets in mini-twistor string theory. We will apply this procedure in 4.6. where we will again encounter our operators $E_{A B}, \bar{E}^{A B}$ and $V_{4}{ }_{B}{ }_{B}$, from 33.9 . More details on bosonization can be found in Appendix A

\section{Mass terms}

We come now to the main new point of our paper — adding mass terms. We can augment the $D=3$ super Yang-Mills Lagrangian with mass terms for the scalars and fermions. The $D=3$ fermions are in the spinor representation 8 of the R-symmetry group $\operatorname{Spin}(7)$. Fermion mass terms are linear in the mass parameter and correspond to operators of the form $M_{a b} \chi_{\alpha}^{a} \chi^{\alpha b}$, where $M_{a b}$ is the mass matrix, and we used the notation of (3.1). These operators are in the symmetric part of the tensor product representation $8 \otimes \mathbf{8}$ of $\operatorname{Spin}(7)$. (It decomposes into the 
irreducible representations $\mathbf{1}+\mathbf{3 5}$.) The $D=3$ scalars are in the vector representation 7 of Spin(7). Scalar mass terms are quadratic in the mass parameter and correspond to operators in the symmetric part of the tensor product representation $\mathbf{7} \otimes \mathbf{7}$. (It decomposes into irreducible representations $\mathbf{1}+\mathbf{2 7}$.) The $D=3$ super-mini-twistor space formalism only exhibits a manifest $S U(4) \subset \operatorname{Spin}(7)$ subgroup of the R-symmetry. This is the subgroup inherited from $D=4$, and the anti-commuting variables $\theta^{A}(A=1, \ldots, 4)$ are in the $\mathbf{4}$ of this $S U(4)$, while the $\partial / \partial \theta^{A}$ derivatives are in the $\overline{4}$ conjugate representation. The operators that correspond to $D=3$ fermion mass terms split into the following $S U(4)$ irreducible representations

$$
(8 \otimes 8)_{S}=(4 \otimes 4)_{S}+(\overline{4} \otimes \overline{4})_{S}+4 \otimes \overline{4}=10+\overline{\mathbf{1 0}}+[\mathbf{1}+15]
$$

Here $(\cdots)_{S}$ denotes the symmetric part of a tensor product, and $\mathbf{1 5}$ is the adjoint representation of $S U(4)$. We will now study the deformations of mini-twistor string theory that correspond to these mass terms. Generating the mass terms in the adjoint representation $\mathbf{1 5}$ is simpler, and we therefore start with those.

\subsection{Dimensional reduction with twists}

A convenient way to achieve a massive theory with mass terms in the $\mathbf{1 5}$ representation is to modify the procedure of dimensional reduction by adding an R-symmetry twist. In 93.7 we obtained massless $D=3$ super Yang-Mills theory from the massless $D=4$ theory by gauging a translation generator $P_{4}$ (in the worldsheet theory). To obtain massive $D=3$ super YangMills theory from the massless $D=4$ theory, we gauge a linear combination of translation and R-symmetry, $P_{4}-\operatorname{tr}(M R)$, where $R$ is the $S U(4)$ R-symmetry charge (realized as a $4 \times 4$ hermitian traceless matrix) and $M$ is a constant $4 \times 4$ hermitian traceless mass-matrix that will end up as the mass-matrix of the $D=3$ fermions. The logic behind this reduction is as follows. Let $\psi_{\alpha}^{A}(A=1, \ldots, 4)$ be a $D=4$ Weyl fermion field, which transforms in the fundamental representation 4 of the R-symmetry group $S U(4)$. Gauging the combination $P_{4}-\operatorname{tr}(M R)$ on the worldsheet corresponds to setting

$$
i \partial_{3} \psi_{\alpha}^{A}=M_{B}^{A} \psi_{\alpha}^{B}
$$

in physical space. The term $\bar{\psi}_{A}^{\dot{\alpha}} \sigma_{\alpha \dot{\alpha}}^{3} \partial_{3} \psi^{A \alpha}$ in the $D=4$ Lagrangian will become a mass term in the $D=3$ dimensionally reduced Lagrangian. The fermion mass matrix will be $M$, and the scalar mass matrix squared will be the anti-symmetric part of $M \otimes M$, which, in the 6 representation of $S U(4)$, is the $6 \times 6$ matrix representative of $M$. 
In twistor space, we augment (3.27) to get

$$
\begin{gathered}
P_{4}-\operatorname{tr}(M R): \quad \delta Z_{1}=\delta Z_{2}=0, \quad \delta Z_{3}=\epsilon Z_{1}, \quad \delta Z_{4}=-\epsilon Z_{2}, \\
\delta \theta^{A}=\epsilon M^{A}{ }_{B} \theta^{B} \quad(A, B=1, \ldots, 4) .
\end{gathered}
$$

Gauging this translation symmetry can be done along the lines that led to (3.10). We get a similar supermanifold that can be covered by two patches $U_{1}, U_{2}$ with transition functions that are a slight modification of (3.10),

$$
z^{\prime}=\frac{1}{z}, \quad w^{\prime}=-\frac{w}{z^{2}}, \quad \theta^{\prime}=\frac{1}{z} e^{i \frac{w}{z} M} \theta
$$

where $\theta$ represents a 4 -component vector, and $\exp [i(w / z) M]$ is a $4 \times 4$ matrix [in the complexification of $S U(4)]$. This is one of our main results. Equation (4.3) describes the target space of a twistor string theory that is, by conjecture, dual to a mass-deformed $D=3$ super Yang-Mills theory with a mass-matrix $M$.

Similarly to the discussion at the end of $\$ 3.7$, we can think of (4.2) as the $r \rightarrow 0$ limit of the discrete orbifold of $\mathbb{C P}^{3 \mid 4}$ by the group $\Gamma_{r, M} \simeq \mathbb{Z}$ generated by

$$
\begin{aligned}
\gamma_{r, M} \equiv e^{2 \pi i r\left(P_{4}-\operatorname{tr}(M R)\right)}: Z_{1} \mapsto Z_{1}, \quad Z_{2} \mapsto Z_{2}, \quad Z_{3} \mapsto Z_{3}+2 \pi r Z_{1}, \\
Z_{4} \mapsto Z_{4}-2 \pi r Z_{2}, \quad \theta \mapsto e^{-2 \pi i r M} \theta,
\end{aligned}
$$

where $\theta$ again denotes a 4-component vector. Physically, (4.4) corresponds to a circle compactification of $D=4$ super Yang-Mills theory with an R-symmetry twist. That is, the scalars and fermions have nonperiodic boundary conditions on the circle. Such compactifications have been studied, for example, in [26]. R-symmetry orbifolds of the $D=4$ theory (without the $P_{4}$ generator) have been recently studied in [27]. For recent developments in worldsheet orbifolds see $[28$.

\subsection{Mini-twistor string theory mass operators}

For any value of the mass matrix $M$, the manifold described by (4.3) is, presumably, the target space of the worldsheet (mini-)twistor string theory that describes the massive deformation studied in 4.1] In particular, for an infinitesimally small $M$, (4.3) describes a small deformation of the complex structure of the super mini-twistor space $\mathbb{T}_{3}$ (defined as $T \mathbb{C P}^{1}$ with four extra anti-commuting coordinates fibered over it). 
In general, a small deformation of the complex structure corresponds to an operator in the worldsheet theory, which is the topological B-model with target space $\mathbb{T}_{3}$. For example, an interesting case was studied in [30] where marginal deformations of the $D=4$ theory [31] 32] 33 . were associated with certain B-model closed string operators. The operators of the topological B-model on a manifold $X$ correspond to the sheaf cohomology classes $H^{p}\left(X, \wedge^{m} T^{(1,0)} X\right)[34$. Locally on $X$, with a choice of complex coordinates $z_{i}\left(i=1, \ldots, \operatorname{dim}_{\mathbb{C}} X\right)$ and their complex conjugates $\bar{z}_{\bar{i}}$, these sheaf cohomology classes can be realized as tensors $V_{\bar{i}_{1} \cdots \bar{i}_{p}}{ }_{1} \cdots j_{m}$ that are anti-symmetric in the $\bar{i}_{s}$ and $j_{t}$ indices. Deformation by the operators with $p=1$ and $m=1$ corresponds to a complex structure deformation. (The target space action that describes these deformation is the Kodaira-Spencer action [35.) This correspondence can be extended, presumably, to supermanifolds and we get the cohomology classes $H^{p \mid q}\left(X, \wedge^{m \mid n} T^{(1,0)} X\right)$. In [30], B-model operators that correspond to elements of $H^{0 \mid 2}\left(X, \wedge^{0 \mid 2} T^{(1,0)} X\right)$, with $X=\mathbb{C P}^{3 \mid 4}$, were identified with cubic deformations of the $D=4, N=4$ super Yang-Mills superpotential. Furthermore, general deformations of holomorphic vector bundles over weighted projective superspaces were recently studied in [22]. The particular complex structure deformation that we need for the mass term is a special case.

Let us now study the $D=3$ super-cohomology classes. First, let us take the mass deformation in the limit of infinitesimal mass matrix $M$. Equation (4.3) teaches us that the infinitesimal change in coordinate transition functions on the intersection $U_{12} \equiv U_{1} \cap U_{2}$ of the two patches $U_{1} \equiv\{z<\infty\}$ and $U_{2} \equiv\left\{z^{\prime}<\infty\right\}$ is

$$
\delta z^{\prime}=0, \quad \delta w^{\prime}=0, \quad \delta \theta^{\prime}=i \frac{w}{z^{2}} M \theta=-i \frac{w^{\prime}}{z^{\prime}} M \theta^{\prime}+O(M)^{2} .
$$

Here, we think of $U_{1}, U_{2}$ as two patches of the undeformed $\left(M^{A}{ }_{B}=0\right) D=3$ twistor supermanifold $\mathbb{T}_{3}$. Equation (4.5) defines a holomorphic vector field on $U_{12}$ whose components are given, in local coordinates, by

$$
\delta z^{\prime} \frac{\partial}{\partial z^{\prime}}+\delta w^{\prime} \frac{\partial}{\partial w^{\prime}}+\delta \theta^{\prime T} \frac{\partial}{\partial \theta^{\prime}}=-i \frac{w^{\prime}}{z^{\prime}} \theta^{\prime T} M^{T} \frac{\partial}{\partial \theta^{\prime}}=i \frac{w}{z} \theta^{T} M^{T} \frac{\partial}{\partial \theta} .
$$

This defines an element of the sheaf cohomology $H^{1 \mid 0}\left(X, \wedge^{0 \mid 1} T^{(1,0)} X\right)$ for $X=\mathbb{T}_{3}$. In fact, $H^{1 \mid 0}\left(X, \wedge^{0 \mid 1} T^{(1,0)} X\right)$ is the space of holomorphic vector fields of the form

$$
V_{B}^{A}\left(z, w, \theta_{1}, \ldots, \theta_{4}\right) \theta_{A} \frac{\partial}{\partial \theta_{B}}
$$

that are defined for $0<z<\infty$ up to the equivalence relation

$$
V_{B}^{A} \sim V_{B}^{A}+\left(V_{\{1\}}\right)_{B}^{A}-\left(V_{\{2\}}\right)_{B}^{A}
$$


where $V_{\{1\}}$ is a holomorphic vector field of a similar form that is defined for all $z<\infty$ (including $z=0$ ) and $V_{\{2\}}$ is similarly defined for all $0<z$ (including $z=\infty$ ). [Recall that in general, given a cover of $X$ by contractible open patches $X=\cup_{\alpha} U_{\alpha}$, an element of $H^{p}\left(X, T^{(1,0)} X\right)$ can be described by a collection of local vector fields, one vector field for each nontrivial intersection of $(p+1)$ patches $U_{\alpha_{1}} \cap \cdots \cap U_{\alpha_{p+1}}$, such that a certain linear relation-the cocycle condition-holds on intersections of $(p+2)$ patches. In our case, there are only two patches, $U_{1}$ and $U_{2}$, and a vector field that is defined on the intersection $U_{1} \cap U_{2}$ defines an element of $H^{1}\left(X, T^{(1,0)} X\right)$.]

The vector field corresponding to (4.6) is

$$
V_{B}^{A}=\frac{w}{z} M_{B}^{A}=-\frac{w^{\prime}}{z^{\prime}} M_{B}^{A} .
$$

This vector field therefore has poles both at $z=0$ and $z^{\prime}=0$.

More generally, the field

$$
\frac{w^{r}}{z^{s}} M_{B}^{A}=\frac{\left(-w^{\prime}\right)^{r}}{z^{\prime 2 r-s}} M_{B}^{A}
$$

has poles both at $z=0$ and $z^{\prime}=0$ only if $0<s<2 r$. For other values of $s$, the vector field can be extended to either $z=0$ or $z=\infty$, and therefore is trivial in cohomology. Let us check for which values of $s, r$ this cohomology class is invariant under translations of the physical $\mathbb{R}^{3}$. The generators of translations were written down in (2.10). Since the field (4.8) is holomorphic, only the holomorphic parts of $P_{1}, P_{2}, P_{3}$ in (2.10) are relevant. We therefore need to check that $z^{k} \partial / \partial w$, for $k=0,1,2$, annihilates (4.8) in cohomology. By the discussion above, this is equivalent to requiring that $0<s-k<2(r-1)$ is not satisfied for any of the values $k=0,1,2$. It is not hard to check that the only solution is $s=r=1$, which is the field from (4.6). Similarly, using equations (2.11) for the rotation generators, it can be checked that (4.6) is rotationally invariant. Thus, the mass deformation (4.6) corresponds to the unique (up to multiplication by a constant) translationally invariant element of $H^{1 \mid 0}\left(X, \wedge^{0 \mid 1} T^{(1,0)} X\right)$.

Alternatively, the cohomology $H^{1 \mid 0}\left(X, \wedge^{0 \mid 1} T^{(1,0)} X\right)$ can be represented by a global differential 1-form with coefficients in $\wedge^{0 \mid 1} T^{(1,0)} X$. The way to convert a sheaf cohomology representative to a global form is to write

$$
V_{B}^{A}=\left(V_{\{1\}}\right)_{B}^{A}-\left(V_{\{2\}}\right)_{B}^{A},
$$

where $V_{\{1\}}$ and $V_{\{2\}}$ are well-defined on $U_{1}$ and $U_{2}$ respectively, but, unlike (4.7), $V_{\{1\}}$ and $V_{\{2\}}$ are not required to be holomorphic (otherwise $V^{A}{ }_{B}$ would be trivial in cohomology). Then, the 1-form $\bar{\partial}\left(V_{\{1\}}\right)_{B}^{A}=\bar{\partial}\left(V_{\{2\}}\right)_{B}^{A}$ (where $\bar{\partial}$ is the Dolbeault differential operator) is globally defined 
and represents the cohomology class. In our case, we can pick an arbitrary differentiable function $f\left(|z|^{2}\right)$ such that $f(0)=1$ and $f(\infty)=0$ and set

$$
\left(V_{\{1\}}\right)_{B}^{A}=\left[1-f\left(|z|^{2}\right)\right] \frac{w}{z} M_{B}^{A}, \quad\left(V_{\{2\}}\right)_{B}^{A}=-f\left(|z|^{2}\right) \frac{w}{z} M_{B}^{A}
$$

Then,

$$
\bar{\partial}\left(V_{\{1\}}\right)_{B}^{A}=\bar{\partial}\left(V_{\{2\}}\right)_{B}^{A}=-f^{\prime}\left(|z|^{2}\right) M_{B}^{A} w d \bar{z}
$$

is a global 1-form that represents the cohomology class. The corresponding B-model operator is easily constructed from this form. It is

$$
V_{(\mathbf{1 5})}=-f^{\prime}(\tilde{Z} \bar{Z}) \bar{\eta}^{\bar{z}} \vartheta_{A} M_{B}^{A} \tilde{W} \Theta^{B}
$$

(The worldsheet fields of the B-model are listed in Table 1) Can the operator corresponding to (4.9) be related by dimensional reduction to a $D=4$ operator? We will work in the coordinates (3.25) for $\mathbb{C P}^{3 \mid 4}$, and define the two patches

$$
U_{1}^{\prime}:=\left\{Z_{1} \neq 0\right\}, \quad U_{2}^{\prime}:=\left\{Z_{2} \neq 0\right\}
$$

Together, $U_{1}^{\prime}$ and $U_{2}^{\prime}$ cover the $D=4$ twistor space (given by the condition that $Z_{1}$ and $Z_{2}$ do not vanish simultaneously). Using (3.6), we calculate

$$
\frac{w}{z}=\frac{\mu^{\dot{1}}}{\lambda_{1}}-\frac{\mu^{\dot{2}}}{\lambda_{2}}=\frac{Z_{3}}{Z_{1}}-\frac{Z_{4}}{Z_{2}}
$$

The element (4.6) can therefore be dimensionally "oxidized" (the inverse of "dimensionally reduced") to the following (super-)vector field on $U_{1}^{\prime} \cap U_{2}^{\prime}$,

$$
V_{m}^{(D=4)}=\left(\frac{Z_{3}}{Z_{1}}-\frac{Z_{4}}{Z_{2}}\right) \theta^{A} M_{A}{ }^{B} \frac{\partial}{\partial \theta^{B}}=\left(\frac{Z_{3}}{Z_{1}} \theta^{A} M_{A}{ }^{B} \frac{\partial}{\partial \theta^{B}}\right)-\left(\frac{Z_{4}}{Z_{2}} \theta^{A} M_{A}^{B} \frac{\partial}{\partial \theta^{B}}\right) .
$$

In the last equality, we have written $V_{m}$ as the difference of two terms, the first of which can be extended to a holomorphic function on the entire $U_{1}^{\prime}$ patch, and the second can be extended to a holomorphic function on the entire $U_{2}^{\prime}$ patch. $V_{m}$ is therefore exact in sheaf cohomology and corresponds to the zero operator. This was to be expected, since the $D=3$ mass terms that we consider in this subsection, which are in $\mathbf{1 5}$ of $S U(4)$, lift to terms of the form $M^{A}{ }_{B} A_{3} \sigma_{\alpha \dot{\alpha}}^{3} \psi_{A}^{\alpha} \bar{\psi}^{B \dot{\alpha}}+$ $O(M)^{2}$ in $D=4$, where $A_{3}$ is the $4^{\text {th }}$ component of the gauge field (counting from 0 ). These terms can be gauged away by an $x_{4}$-dependent gauge transformation. 


\subsection{The 10 and $\overline{\mathbf{1 0}}$ mass terms.}

We still have to analyze the remaining mass terms from (4.1). These are the terms in the representations 10, their complex conjugates in $\overline{\mathbf{1 0}}$, and the representation $\mathbf{1}$. We will discuss the singlet $\mathbf{1}$ separately, and concentrate on the $\mathbf{1 0}$ and $\overline{\mathbf{1 0}}$ first.

First, let us ask whether these $D=3$ mass terms can be derived by dimensional reduction of $D=4$ mass terms. The answer is yes! Looking back at (4.1), we see that, unlike the mass terms in $\mathbf{1 5}$ that were discussed in 4.1 , the mass terms in $\mathbf{1 0}$ and $\overline{\mathbf{1 0}}$ can indeed be gotten by dimensional reduction of $D=4$ mass terms. This is because $D=4$ mass terms are of the form $\epsilon^{\alpha \beta} \psi_{\alpha}^{A} \psi_{\beta}^{B}$ and $\epsilon^{\dot{\alpha} \dot{\beta}} \bar{\psi}_{\dot{\alpha} A} \bar{\psi}_{\dot{\beta} B}$ but cannot involve $\psi_{\alpha}^{A} \bar{\psi}_{\dot{\alpha} B}$, since spinors of different chirality in $D=4$ cannot be contracted in a Lorentz invariant manner. After dimensional reduction to $D=3$, however, both spinors become the same representation. Thus, the $D=3$ mass terms in $\mathbf{1 0}$ and $\overline{\mathbf{1 0}}$ can be derived from dimensionally reduced $D=4$ mass terms, but the mass terms in $\mathbf{1 5}$ have to be derived by twisting as in $\$ 4.1$

So what are the $D=4$ operators that can give such mass terms upon dimensional reduction? These are closed string vertex operators of the B-model twistor string theory of $D=4$ super YangMills. It has been argued in [4 14 36 that they correspond to fields of $D=4$ superconformal supergravity. In particular, Table 1 in $\S 4.2$ of [14] lists the physical states of that theory. And indeed, among these fields we find two that have the right quantum numbers. In the notation of [14], these are $E_{(A B)}(A, B=1, \ldots 4$ are $S U(4)$ indices $)$ in $\overline{\mathbf{1 0}}$, and $\bar{E}^{(A B)}$ in $\mathbf{1 0}$. The conformal dimensions of these fields can be read off from their $U(1)_{R}$ charge, which is $Q=-2$ (and is also listed in Table 1 of [14]). Their conformal dimensions are $\Delta=D+(3 / 2) Q=1$, and this is consistent with a fermionic mass term.

Now let us write down the vertex operators for the dimensionally reduced $D=3$ mass terms in the 10 representation. The $\bar{E}^{A B}$ operators correspond to linear combinations of vector fields of the form

$$
\frac{w^{r}}{z^{s}} M^{A B} \epsilon_{A C D E} \theta^{C} \theta^{D} \theta^{E} \frac{\partial}{\partial \theta^{B}},
$$

defined on the intersection $U_{1} \cap U_{2}$ (i.e., $0<|z|<\infty$ ), where $r, s$ are integers. By the same arguments that follow (4.8) we conclude that only $r=0$ and $s=1$ describes a nonzero Poincaré invariant operator. Converting from sheaf to Dolbeault $\bar{\partial}$-cohomology, we find the operator

$$
V_{(\mathbf{1 0})}=-\frac{1}{6} f^{\prime}(\tilde{Z} \bar{Z}) \bar{\eta}^{\bar{z}} M^{A B} \epsilon_{A C D E} \Theta^{C} \Theta^{D} \Theta^{E} \vartheta_{B} .
$$


Here, $f\left(|z|^{2}\right)$ is, as in (4.9), an arbitrary function that satisfies $f(0)=1$ and $f(\infty)=0$.

\subsection{Chiral $D=4$ mass terms}

Let us make a brief digression to check that $\bar{E}^{A B}$ indeed corresponds to a mass term in $D=4$. We hope to present a more complete analysis elsewhere [53].

The mass-deformed $N=4$ super Yang-Mills theory that, we claim, corresponds to turning on an $\bar{E}^{A B} \mathrm{VEV}$ is nonstandard. It is the CPT violating theory that one obtains by giving a mass term only to the positive helicity spinors of $D=4, N=4$ super Yang-Mills theory. This is a perturbation of the form $M^{A B} \psi_{\alpha A} \psi_{B}^{\alpha}$, where $\psi_{\alpha A}$ denote, as before, the $D=4$ fermions in the $\overline{4}$ of $S U(4)$.

In momentum space, the free massive Dirac equation now reads

$$
p_{\alpha \dot{\alpha}} \psi^{\alpha A}=M^{A B} \bar{\psi}_{\dot{\alpha} B}, \quad p_{\alpha \dot{\alpha}} \bar{\psi}_{A}^{\dot{\alpha}}=0
$$

Thus, the 4-momentum $p_{\alpha \dot{\alpha}}$ is still lightlike, and we can decompose it as in the massless case,

$$
p_{\alpha \dot{\alpha}}=\lambda_{\alpha} \tilde{\lambda}_{\dot{\alpha}}
$$

The general solution to (4.14) is of the form

$$
\bar{\psi}_{\dot{\alpha} A}=\tilde{\lambda}_{\dot{\alpha}} \widetilde{\varrho}_{A}, \quad \psi_{\alpha}^{A}=\lambda_{\alpha} \varrho^{A}+M^{A B} \eta_{\alpha} \widetilde{\varrho}_{B},
$$

where $\widetilde{\varrho}_{A}(\lambda, \tilde{\lambda})$ and $\varrho^{A}(\lambda, \tilde{\lambda})$ are arbitrary functions, and $\eta_{\alpha}$ is any $(\lambda$-dependent) solution to the linear equation

$$
\eta_{\alpha} \lambda^{\alpha}=1
$$

There is, of course, a family of solutions to this equation. Given a solution $\eta_{\alpha}$ and an arbitrary function $\zeta(\lambda, \tilde{\lambda})$, the following is also a solution to (4.16):

$$
\eta_{\alpha}^{\prime}:=\eta_{\alpha}+\lambda_{\alpha} \zeta
$$

If we choose $\eta_{\alpha}^{\prime}$ instead of $\eta_{\alpha}$ as the solution to (4.16), we can preserve the physical wavefunctions (4.15) by setting

$$
\widetilde{\varrho}_{A}:=\widetilde{\varrho}_{A}, \quad \varrho^{A}:=\varrho^{A}-M^{A B} \zeta \widetilde{\varrho}_{B},
$$

so that

$$
\bar{\psi}_{\dot{\alpha} A}=\tilde{\lambda}_{\dot{\alpha}} \widetilde{\varrho}_{A}, \quad \psi_{\alpha}^{A}=\lambda_{\alpha} \varrho^{A}+M^{A B} \widetilde{\varrho}_{B} \eta_{\alpha}^{\prime}
$$


An example of a solution to (4.16) is given by $\eta_{1}=1 / \lambda^{1}$ and $\eta_{2}=0$. It is well-defined on the patch of $\lambda$-space where $\lambda^{1} \neq 0$. Similarly, on the patch where $\lambda^{2} \neq 0$ we can take $\eta_{2}^{\prime}=1 / \lambda^{2}$ and $\eta_{1}^{\prime}=0$, so that on the intersection of the two patches, where both components of $\lambda$ are nonzero, we have $\eta_{\alpha}^{\prime}=\eta_{\alpha}-\epsilon_{\alpha \beta} \lambda^{\beta} /\left(\lambda^{1} \lambda^{2}\right)$. If we choose to work with $\eta$ on the patch $\lambda^{1} \neq 0$ and with $\eta^{\prime}$ on the patch $\lambda^{2} \neq 0$, we need the transition relations

$$
\varrho^{\prime A}=\varrho^{A}+\frac{1}{\lambda^{1} \lambda^{2}} M^{A B} \widetilde{\varrho}_{B}, \quad \widetilde{\varrho}_{A}=\widetilde{\varrho}_{A} .
$$

External fermions in scattering amplitudes are described by wave-functions of the form (4.15). These wave-functions can be twistor-transformed as usual,

$$
\widehat{\varrho}^{A}(\lambda, \mu):=\int d^{2} \tilde{\lambda} e^{i \mu_{\dot{\alpha}} \tilde{\lambda}^{\dot{\alpha}}} \varrho^{A}(\lambda, \tilde{\lambda}), \quad \widehat{\widetilde{\varrho}}_{A}(\lambda, \mu):=\int d^{2} \tilde{\lambda} e^{i \mu_{\dot{\alpha}} \tilde{\lambda}^{\dot{\alpha}}} \widetilde{\varrho}_{A}(\lambda, \tilde{\lambda}) .
$$

The freedom (4.18) extends to the twistor transforms

$$
\widehat{\widetilde{\varrho}}_{A}:=\widehat{\widetilde{\varrho}}_{A}, \quad \widehat{\varrho}^{A}:=\widehat{\varrho}^{A}-M^{A B} \widehat{\zeta} \cdot \widehat{\widetilde{\varrho}}_{B}
$$

where $\widehat{\zeta} \cdot \widehat{\widetilde{\varrho}}_{A}$ denotes the convolution of $\widehat{\widetilde{\varrho}}_{A}$ with the twistor transform of $\zeta$, and the convolution is taken with respect to $\mu_{\dot{\alpha}}$. Note, however, that the equivalence (4.18) can be generalized to $\zeta$ 's that are operator functions of $\lambda_{\alpha}, \tilde{\lambda}_{\dot{\alpha}}$ and $\partial / \partial \tilde{\lambda}_{\dot{\alpha}}$. For the special case that $\zeta$ is of the form $\zeta\left(\lambda_{\alpha}, i \partial / \partial \tilde{\lambda}_{\dot{\alpha}}\right)$ the convolution becomes an ordinary product, and (4.20) becomes

$$
\widehat{\widetilde{\varrho}}_{A}:=\widehat{\widetilde{\varrho}}_{A}, \quad \widehat{\varrho}^{A}:=\widehat{\varrho}^{A}-M^{A B} \zeta \widehat{\widetilde{\varrho}}_{B},
$$

where $\zeta(\lambda, \mu)$ is a function on twistor space.

Following similar steps as in the appendix of [4], we take the twistor transforms (4.19), plug them into (4.15), and integrate over $\lambda$ and $\tilde{\lambda}$ to convert from momentum-space back to coordinate space. We perform the $\lambda_{\alpha}$-integrals by gauge-fixing $\lambda^{1}=1$ and integrating $z \equiv \lambda^{2}$ over a path $C$ around the origin.

$$
\begin{aligned}
\bar{\psi}_{\dot{\alpha} A}(x) & =\frac{1}{2 \pi} \oint_{C} d \lambda^{2} \int d^{2} \tilde{\lambda} \int d^{2} \mu e^{i x_{\alpha \dot{\alpha}} \lambda^{\alpha} \tilde{\lambda}^{\dot{\alpha}}-i \mu_{\dot{\alpha}} \tilde{\lambda}^{\dot{\alpha}}} \tilde{\lambda}_{\dot{\alpha}} \widehat{\widetilde{\varrho}}_{A}(\lambda, \mu)=\left.\frac{1}{2 \pi i} \oint_{C} d z \frac{\partial \widehat{\widetilde{\varrho}}_{A}}{\partial \mu_{\dot{\alpha}}}\right|_{\left(\lambda^{\alpha}, x_{\alpha \dot{\alpha}} \lambda^{\alpha}\right)}, \\
\psi_{\alpha}^{A}(x) & =\frac{1}{2 \pi} \oint_{C} d \lambda^{2} \int d^{2} \tilde{\lambda} \int d^{2} \mu e^{i x_{\alpha \dot{\alpha}} \lambda^{\alpha} \tilde{\lambda}^{\dot{\alpha}}-i \mu_{\dot{\alpha}} \tilde{\lambda}^{\dot{\alpha}}}\left[\lambda_{\alpha} \widehat{\varrho}^{A}(\lambda, \mu)+M^{A B} \eta_{\alpha}(\lambda) \widehat{\widetilde{\varrho}}_{B}(\lambda, \mu)\right] \\
& =\frac{1}{2 \pi} \oint_{C} d z\left[\lambda_{\alpha} \widehat{\varrho}^{A}\left(\lambda^{\alpha}, x_{\alpha \dot{\alpha}} \lambda^{\alpha}\right)+M^{A B} \eta_{\alpha}(\lambda) \widehat{\widetilde{\varrho}}_{B}\left(\lambda^{\alpha}, x_{\alpha \dot{\alpha}} \lambda^{\alpha}\right)\right]
\end{aligned}
$$

where

$$
\left(\lambda^{1}, \lambda^{2}\right) \equiv(1, z), \quad\left(\eta_{1}, \eta_{2}\right) \equiv(1,0)
$$


Now let us analyze the coupling of the closed string B-model mode $\bar{E}^{A B}$ to the open string modes. In general, the coupling of closed string B-model modes to open string modes has been extensively studied. (See 42, 43] 44] and [30] for example.) Turning on a VEV for $\bar{E}^{A B}$ corresponds to a perturbation of the complex structure of the super-manifold target space $\mathbb{C P}^{3 / 4}$. We will denote by $X^{i}(i=1, \ldots, 4)$ and $\Psi^{A}(A=1, \ldots, 4)$ the homogeneous coordinates on $\mathbb{C P}^{3 \mid 4}$. (In [4], $X^{i}$ is denoted by $Z^{I}$, but we use the symbols $Z^{1}, Z^{2}$ to denote some of the projective coordinates of $W \mathbb{C P}^{1,1 \mid 2}$.) We will cover the $D=4$ twistor space $\mathbb{C P}^{3 \mid 4} \backslash \mathbb{C P}^{1 \mid 4}$ with the two patches

$$
U_{1}:=\left\{X^{1} \neq 0\right\}, \quad \text { and } \quad U_{2}:=\left\{X^{2} \neq 0\right\}
$$

On the patch $U_{1}$ where $X^{1} \neq 0$, we can rescale the projective coordinates and set $X^{1}=1$. Then $X^{2}, X^{3}, X^{4}$ and the $\Psi^{A}$ are the independent coordinates. The good coordinates on the patch $U_{2}$, where $X^{2} \neq 0$, are then

$$
\frac{1}{X^{2}}, \frac{X^{3}}{X^{2}}, \frac{X^{4}}{X^{2}}, \frac{\Psi^{1}}{X^{2}}, \ldots, \frac{\Psi^{4}}{X^{2}}
$$

On the patch $X^{1} \neq 0$, the $\mathrm{VEV}\left\langle\bar{E}^{A B}\right\rangle=M^{A B}$ corresponds to the local holomorphic vector field

$$
\frac{1}{6 X^{2}} M^{A B} \epsilon_{A C D E} \Psi^{C} \Psi^{D} \Psi^{E} \frac{\partial}{\partial \Psi^{B}}
$$

[see (4.12) with $r=0$ and $s=1$ ]. This vector field, in turn, corresponds to a (super-)complex structure deformation of the B-model target space $\mathbb{C P}^{3 \mid 4}$.

Formula (4.16) of [4] gives us the component expansion of the B-model super 1-form field as follows,

$$
\begin{aligned}
\mathcal{A}(X, \bar{X}, \Psi)= & d \bar{X}^{\bar{i}}\left(A_{\bar{i}}+\Psi^{A} \chi_{\bar{i} A}+\frac{1}{2} \Psi^{A} \Psi^{B} \phi_{\bar{i} A B}\right. \\
& \left.+\frac{1}{6} \epsilon_{A B C D} \Psi^{A} \Psi^{B} \Psi^{C} \widetilde{\chi}_{\bar{i}}^{D}+\frac{1}{24} \epsilon_{A B C D} \Psi^{A} \Psi^{B} \Psi^{C} \Psi^{D} G_{\bar{i}}\right)
\end{aligned}
$$

where $A_{\bar{i}}, \chi_{\bar{i} A}, \phi_{\bar{i} A B}, \widetilde{\chi}_{\bar{i}}^{D}$ and $G_{\bar{i}}$ are functions of $X$ and $\bar{X}$. The classical action is given by equation (4.18) of [4],

$$
I=\frac{1}{2} \int \Omega \wedge\left(\mathcal{A} \wedge \bar{\partial} \mathcal{A}+\frac{2}{3} \mathcal{A} \wedge \mathcal{A} \wedge \mathcal{A}\right)
$$

The equations of motion of this holomorphic Chern-Simons theory, to first order, state that $\mathcal{A}$ is an element of $\bar{\partial}$-cohomology

$$
\bar{\partial} \mathcal{A}=0, \quad \mathcal{A} \sim \mathcal{A}+\bar{\partial} \Lambda
$$


where $\Lambda$ is an arbitrary function.

For our purposes, however, it is more convenient to let $\mathcal{A}$ be an element of Čech cohomology rather than $\bar{\partial}$-cohomology. For ordinary manifolds these cohomologies are equivalent, but the advantage of Čech cohomology is that we work with holomorphic functions. For supermanifolds the situation is more complicated, and we refer the reader to [37] [21] [22] for further details. ${ }^{7}$

In Čech cohomology, which is a special case of sheaf cohomology, the B-model field is represented by a holomorphic function $\mathcal{A}$ that is defined on the intersection of patches $U_{1} \cap U_{2}$. This is in contrast to $\bar{\partial}$-cohomology for which $\mathcal{A}$ was a $(1,0)$-form and was not necessarily represented by holomorphic functions, but was defined on the entire manifold $U_{1} \cup U_{2}$. In Čech cohomology, there is an equivalence relation

$$
\mathcal{A} \sim \mathcal{A}+\Lambda_{1}+\Lambda_{2}
$$

where $\Lambda_{1}$ and $\Lambda_{2}$ are holomorphic functions on $U_{1}$ and $U_{2}$, respectively.

How does the infinitesimal complex structure deformation that corresponds to (4.24) change the Čech cohomology class $\mathcal{A}$ ? The infinitesimal complex structure deformation can be interpreted as a change in the holomorphic transition functions between the patch $U_{1}$ and the patch $U_{2}$. In the deformed space, (4.23) are no longer good coordinates on $U_{2}$. Instead, a good set of coordinates is

$$
\frac{1}{X^{2}}, \frac{X^{3}}{X^{2}}, \frac{X^{4}}{X^{2}}, \frac{\Psi^{A}}{X^{2}}+\frac{1}{6\left(X^{2}\right)^{2}} M^{A B} \epsilon_{B C D E} \Psi^{C} \Psi^{D} \Psi^{E} \quad A=1, \ldots, 4,
$$

where we used (4.24). Thus, the Čech equivalence relation (4.27) has to be modified to

$$
\begin{aligned}
\mathcal{A}\left(X^{2}, X^{3}, X^{4},\left\{\Psi^{A}\right\}\right) & \sim \mathcal{A}\left(X^{2}, X^{3}, X^{4},\left\{\Psi^{A}\right\}\right)+\Lambda_{1}\left(X^{2}, X^{3}, X^{4},\left\{\Psi^{A}\right\}\right) \\
& +\Lambda_{2}\left(\frac{1}{X^{2}}, \frac{X^{3}}{X^{2}}, \frac{X^{4}}{X^{2}},\left\{\frac{\Psi^{A}}{X^{2}}+\frac{1}{6\left(X^{2}\right)^{2}} M^{A B} \epsilon_{B C D E} \Psi^{C} \Psi^{D} \Psi^{E}\right\}\right),
\end{aligned}
$$

where $\Lambda_{1}$ and $\Lambda_{2}$ are holomorphic functions of their variables. Let us expand $\mathcal{A}$ in components, similarly to (4.25).

$$
\begin{aligned}
\mathcal{A}= & A\left(X^{2}, X^{3}, X^{4}\right)+\Psi^{A} \chi_{A}\left(X^{2}, X^{3}, X^{4}\right)+\frac{1}{2} \Psi^{A} \Psi^{B} \phi_{A B}\left(X^{2}, X^{3}, X^{4}\right) \\
& +\frac{1}{6} \epsilon_{A B C D} \Psi^{A} \Psi^{B} \Psi^{C} \widetilde{\chi}^{D}\left(X^{2}, X^{3}, X^{4}\right)+\frac{1}{24} \epsilon_{A B C D} \Psi^{A} \Psi^{B} \Psi^{C} \Psi^{D} G\left(X^{2}, X^{3}, X^{4}\right),
\end{aligned}
$$

where $A, \chi_{A}, \phi_{A B}, \widetilde{\chi}_{D}$ and $G$ are holomorphic functions of $X^{2}, X^{3}, X^{4}$ and are defined on $U_{1} \cap U_{2}$, that is, for $X^{2} \neq 0$ and $X^{2} \neq \infty$. (Since confusion is not likely to arise, we use the same notation

\footnotetext{
${ }^{7}$ We are grateful to Alexander Popov, Christian Sämann and Martin Wolf for pointing this to us.
} 
for the component fields in Čech cohomology as in $\bar{\partial}$-cohomology.) The freedom to add $\Lambda_{1}$ in (4.29) implies that each of the component fields can be augmented by a holomorphic function of $X^{2}, X^{3}, X^{4}$ that is nonsingular at $X^{2}=0$. Thus, the Čech cohomology classes of the component fields are only sensitive to the singular behavior of the fields at $X^{2}=0$.

The freedom to add $\Lambda_{2}$ in (4.29) is now more complicated. Let the component expansion of $\Lambda_{2}$ be

$$
\Lambda_{2}\left(y^{2}, y^{3}, y^{4},\left\{\theta^{A}\right\}\right)=\varpi+\theta^{A} \zeta_{A}+\frac{1}{2 !} \theta^{A} \theta^{B} \varsigma_{A B}+\frac{1}{3 !} \epsilon_{A B C D} \theta^{A} \theta^{B} \theta^{C} v^{D}+\frac{1}{4 !} \epsilon_{A B C D} \theta^{A} \theta^{B} \theta^{C} \theta^{D} \kappa
$$

where $\varpi, \varsigma, v, \kappa$ are holomorphic functions of their generic variables $y^{2}, y^{3}, y^{4}$. Expanding (4.29) in components, we now find the equivalence relations

$$
\begin{aligned}
& \chi_{A}\left(X^{2}, X^{3}, X^{4}\right) \sim \chi_{A}\left(X^{2}, X^{3}, X^{4}\right)+\frac{1}{X^{2}} \zeta_{A}\left(\frac{1}{X^{2}}, \frac{X^{3}}{X^{2}}, \frac{X^{4}}{X^{2}}\right) \\
& \tilde{\chi}^{A}\left(X^{2}, X^{3}, X^{4}\right) \sim \widetilde{\chi}^{A}\left(X^{2}, X^{3}, X^{4}\right)+\frac{1}{\left(X^{2}\right)^{3}} v^{A}\left(\frac{1}{X^{2}}, \frac{X^{3}}{X^{2}}, \frac{X^{4}}{X^{2}}\right)-\frac{1}{\left(X^{2}\right)^{2}} M^{A B} \zeta_{B}\left(\frac{1}{X^{2}}, \frac{X^{3}}{X^{2}}, \frac{X^{4}}{X^{2}}\right)
\end{aligned}
$$

for the fermions, and

$$
\begin{aligned}
A\left(X^{2}, X^{3}, X^{4}\right) & \sim A\left(X^{2}, X^{3}, X^{4}\right)+\varpi\left(\frac{1}{X^{2}}, \frac{X^{3}}{X^{2}}, \frac{X^{4}}{X^{2}}\right), \\
\phi_{A B}\left(X^{2}, X^{3}, X^{4}\right) & \sim \phi_{A B}\left(X^{2}, X^{3}, X^{4}\right)+\frac{1}{\left(X^{2}\right)^{2}} \varsigma_{A B}\left(\frac{1}{X^{2}}, \frac{X^{3}}{X^{2}}, \frac{X^{4}}{X^{2}}\right), \\
G\left(X^{2}, X^{3}, X^{4}\right) & \sim G\left(X^{2}, X^{3}, X^{4}\right)+\frac{1}{\left(X^{2}\right)^{4}} \kappa\left(\frac{1}{X^{2}}, \frac{X^{3}}{X^{2}}, \frac{X^{4}}{X^{2}}\right)
\end{aligned}
$$

for the scalars, where we have used the symmetry of $M^{A B}$ in the $A, B$ indices. We see that only the equivalence relation for the field $\widetilde{\chi}^{A}$ is modified. This field is the twistor transform of the $h=-1 / 2$ helicity spinor $\psi_{\alpha}^{A}$. We would now like to relate the modified equivalence relation to the solution to the massive Dirac equation (4.15).

Let us first recall the origin of the equivalence relations (4.32) in the massless case $\left(M^{A B}=0\right)$. It was explained in the appendix of [4] that the physical wave-functions are recovered from $\widetilde{\chi}^{A}$ and $\chi_{A}$ by a Cauchy integral along a path $C$ that encircles the origin,

$$
\begin{aligned}
& \psi_{\alpha}^{A}(x)=\frac{1}{2 \pi i} \oint_{C} \lambda_{\alpha} \widetilde{\chi}^{A}\left(z, x_{1 \dot{1}}+x_{2 \dot{1}} z, x_{1 \dot{2}}+x_{2 \dot{2}} z\right) d z \\
& \psi_{A}^{\dot{\alpha}}(x)=\frac{1}{2 \pi i} \oint_{C} \frac{\partial}{\partial x_{1 \dot{\alpha}}} \chi_{A}\left(z, x_{1 \dot{1}}+x_{2 \mathrm{i}} z, x_{1 \dot{2}}+x_{2 \dot{2}} z\right) d z
\end{aligned}
$$


where we have fixed the rescaling freedom by

$$
\lambda^{1} \equiv 1, \quad \lambda^{2} \equiv z
$$

[Equations (4.34) are the analogs of Whittaker's formula (2.1) for spinor fields in $D=4$.] For $M^{A B}=0$, the equivalence relation (4.32) can be written in this patch as

$$
\begin{aligned}
& \widetilde{\chi}^{A}\left(z, x_{1 \dot{1}}+x_{2 \dot{1}} z, x_{1 \dot{2}}+x_{2 \dot{2}} z\right) \sim \widetilde{\chi}^{A}\left(z, x_{1 \dot{1}}+x_{2 \dot{1}} z, x_{1 \dot{2}}+x_{2 \dot{2}} z\right)+\frac{1}{z^{3}} v^{A}\left(\frac{1}{z}, \frac{x_{1 \dot{1}}}{z}+x_{2 \dot{1}}, \frac{x_{1 \dot{2}}}{z}+x_{2 \dot{2}}\right), \\
& \chi_{A}\left(z, x_{1 \dot{1}}+x_{2 \dot{1}} z, x_{1 \dot{2}}+x_{2 \dot{2}} z\right) \sim \chi_{A}\left(z, x_{1 \dot{1}}+x_{2 \dot{1}} z, x_{1 \dot{2}}+x_{2 \dot{2}} z\right)+\frac{1}{z} \zeta_{A}\left(\frac{1}{z}, \frac{x_{1 \dot{1}}}{z}+x_{2 \dot{1}}, \frac{x_{1 \dot{2}}}{z}+x_{2 \dot{2}}\right) .
\end{aligned}
$$

These equivalences hold because of the identities

$$
\begin{aligned}
& 0=\frac{1}{2 \pi i} \oint_{C} \lambda_{\alpha} \frac{1}{z^{3}} v^{A}\left(\frac{1}{z}, \frac{x_{1 \dot{1}}}{z}+x_{2 \dot{1}}, \frac{x_{1 \dot{2}}}{z}+x_{2 \dot{2}}\right) d z \\
& 0=\frac{1}{2 \pi i} \oint_{C} \frac{1}{z^{2}} \frac{\partial}{\partial x_{2 \dot{\alpha}}} \zeta_{A}\left(\frac{1}{z}, \frac{x_{1 \dot{1}}}{z}+x_{2 \dot{1}}, \frac{x_{1 \dot{2}}}{z}+x_{2 \dot{2}}\right) d z
\end{aligned}
$$

that can be derived by deforming the contour of integration into a circle of radius $|z| \rightarrow \infty$. (Note that $\lambda_{\alpha}$ behaves at worst like $z$, and $v^{A}$ at worst like a constant.) In the second identity in (4.37) we have set $\partial \zeta_{A} / \partial x_{1 \dot{\alpha}}=\left(\partial \zeta_{A} / \partial x_{2 \dot{\alpha}}\right) / z$.

For the massless case $M^{A B}=0$, equation (4.34) is the same as (4.22) with

$$
\widehat{\widetilde{\varrho}}^{A} \equiv \widetilde{\chi}^{A}, \quad \widehat{\varrho}_{A} \equiv \chi_{A}
$$

Now let us turn to the massive case. Rewriting the Dirac wave-functions (4.22) in terms of the B-model fields (4.38), we get

$$
\begin{aligned}
& \psi_{\alpha}^{A}(x)=\frac{1}{2 \pi i} \oint_{C}\left[\lambda_{\alpha} \widetilde{\chi}^{A}\left(z, x_{1 \dot{1}}+x_{2 \dot{1}} z, x_{1 \dot{2}}+x_{2 \dot{2}} z\right)+M^{A B} \eta_{\alpha} \chi_{B}\left(z, x_{1 \dot{1}}+x_{2 \dot{1}} z, x_{1 \dot{2}}+x_{2 \dot{2}} z\right)\right] d z \\
& \psi_{A}^{\dot{\alpha}}(x)=\frac{1}{2 \pi i} \oint_{C} \frac{\partial}{\partial x_{1 \dot{\alpha}}} \chi_{A}\left(z, x_{1 \dot{1}}+x_{2 \dot{1}} z, x_{1 \dot{2}}+x_{2 \dot{2}} z\right) d z
\end{aligned}
$$

where

$$
\left(\lambda^{1}, \lambda^{2}\right) \equiv(1, z), \quad\left(\eta_{1}, \eta_{2}\right) \equiv(1,0)
$$

The equivalences (4.36) now have to be modified to

$$
\begin{aligned}
& \tilde{\chi}^{A}\left(z, x_{1 \dot{1}}+x_{2 \dot{1}} z, x_{1 \dot{2}}+x_{2 \dot{2}} z\right) \sim \tilde{\chi}^{A}\left(z, x_{1 \dot{1}}+x_{2 \dot{1}} z, x_{1 \dot{2}}+x_{2 \dot{2}} z\right) \\
& \quad+\frac{1}{z^{3}} v^{A}\left(\frac{1}{z}, \frac{x_{1 \dot{1}}}{z}+x_{2 \dot{1}}, \frac{x_{1 \dot{2}}}{z}+x_{2 \dot{2}}\right)+\frac{1}{z^{2}} M^{A B} \zeta_{B}\left(\frac{1}{z}, \frac{x_{1 \dot{1}}}{z}+x_{2 \dot{1}}, \frac{x_{1 \dot{2}}}{z}+x_{2 \dot{2}}\right), \\
& \quad \chi_{A}\left(z, x_{1 \dot{1}}+x_{2 \dot{1}} z, x_{1 \dot{2}}+x_{2 \dot{2}} z\right) \\
& \quad \sim \chi_{A}\left(z, x_{1 \dot{1}}+x_{2 \dot{1}} z, x_{1 \dot{2}}+x_{2 \dot{2}} z\right)+\frac{1}{z} \zeta_{A}\left(\frac{1}{z}, \frac{x_{1 \dot{1}}}{z}+x_{2 \dot{1}}, \frac{x_{1 \dot{2}}}{z}+x_{2 \dot{2}}\right) .
\end{aligned}
$$


Indeed, these transformations leave the Cauchy integrals (4.39) invariant. Moreover, the Čech equivalence relations (4.32), in the form (4.40), determine the form of the Cauchy integrals (4.39), with $\eta_{\alpha}$ determined up to the ambiguity (4.17). Thus we have shown that a VEV for $\bar{E}^{A B}$ corresponds to a chiral mass term.

\subsection{Mass terms in Berkovits's model}

We will now study the counterparts of the mass operators (4.10) and (4.13) in Berkovits's twistor string theory. We will work with the worldsheet fields from \$3.8, Berkovits and Witten explained in [14] how to convert B-model operators to operators in Berkovits's model. Adapted to our $D=3$ setting, the procedure is as follows. We start with a holomorphic vector field on mini-twistor space $\mathbb{T}_{3}$. It can be represented in homogeneous coordinates as

$$
f^{\prime}:=f^{i}\left(Z^{1}, Z^{2}, W, \Theta\right) \frac{\partial}{\partial Z^{i}}+f^{W}\left(Z^{1}, Z^{2}, W, \Theta\right) \frac{\partial}{\partial W}+f^{A}\left(Z^{1}, Z^{2}, W, \Theta\right) \frac{\partial}{\partial \Theta^{A}},
$$

subject to the equivalence relation

$$
f^{\prime} \simeq f^{\prime}+\Lambda\left(Z^{1}, Z^{2}, W, \Theta\right)\left(Z^{i} \frac{\partial}{\partial Z^{i}}+2 W \frac{\partial}{\partial W}+\Theta^{A} \frac{\partial}{\partial \Theta^{A}}\right)
$$

This vector field is converted to a Berkovits vertex operator

$$
\mathcal{V}=Y_{i} f^{i}+U f^{W}+\Upsilon_{A} f^{A}
$$

In particular, starting with a B-model operator that is represented as a holomorphic vector field

$$
f:=f^{z}(z, w, \theta) \frac{\partial}{\partial z}+f^{w}(z, w, \theta) \frac{\partial}{\partial w}+f^{A}(z, w, \theta) \frac{\partial}{\partial \theta^{A}}
$$

we can get the worldsheet vertex operator by lifting (4.42) to $\mathbb{C}^{4 \mid 4}$ by setting $Z^{1}=1, Z^{2}=z$ and $W=w$.

$$
\mathcal{V}=Y_{2} f^{z}\left(\frac{Z^{2}}{Z^{1}}, \frac{W}{\left(Z^{1}\right)^{2}}, \frac{\Theta}{Z^{1}}\right)+U f^{w}\left(\frac{Z^{2}}{Z^{1}}, \frac{W}{\left(Z^{1}\right)^{2}}, \frac{\Theta}{Z^{1}}\right)+\Upsilon_{A} f^{A}\left(\frac{Z^{2}}{Z^{1}}, \frac{W}{\left(Z^{1}\right)^{2}}, \frac{\Theta}{Z^{1}}\right)
$$

This is an open string vertex operator in Berkovits's model. The vector field (4.42) is required to preserve the holomorphic superform on mini-twistor space

$$
\Omega=d w \wedge d z \wedge d \theta^{1} \cdots d \theta^{4}
$$

This is a condition that descends from a similar condition in $D=4$ [14]. 
Applying this Berkovits-Witten prescription to (4.10) we get our first ansatz for the Berkovitsmodel mass operators

$$
V_{(15)} \rightarrow \mathcal{V}_{(15)}=\frac{W}{Z^{1} Z^{2}} \Upsilon_{A} M_{B}^{A} \Theta^{B} .
$$

The factor of $Z^{1} Z^{2}$ in the denominator of (4.43) might appear strange at first, but it can be handled by bosonization. We have discussed a similar issue at the end of 3.9 and more details can be found in Appendix $\mathrm{A}$.

To gain more insight, we will now derive (4.43) by directly gauging translations with an R-symmetry twist (4.2) in the world-sheet action (3.301). We therefore augment the covariant derivative (3.34) according to the modified gauge transformation (4.2),

$$
\nabla_{\overline{\mathfrak{z}}} Z_{L}^{3}=\partial_{\overline{\mathfrak{z}}} Z_{L}^{3}-A_{\overline{\mathfrak{z}}} Z_{L}^{3}-\widetilde{B}_{\overline{\mathfrak{z}}} Z_{L}^{1}, \quad \nabla_{\overline{\mathfrak{z}}} Z_{L}^{4}=\partial_{\overline{\mathfrak{z}}} Z_{L}^{4}-A_{\overline{\mathfrak{z}}} Z_{L}^{4}+\widetilde{B}_{\overline{\mathfrak{z}}} Z_{L}^{2}, \quad \nabla_{\overline{\mathfrak{z}}} \Theta_{L}^{A}=\partial_{\overline{\mathfrak{z}}} \Theta_{L}^{A}-i \widetilde{B}_{\overline{\mathfrak{z}}} M_{B}^{A} \Theta^{B},
$$

and similarly for the right-moving fields. Inserting these covariant derivatives into the action (3.30) and integrating out the nondynamical gauge fields $\widetilde{B}_{\mathfrak{z}}, \widetilde{B}_{\bar{z}}$, we get the modified constraint

$$
j_{m}(\mathfrak{z}):=Y_{3} Z^{1}-Y_{4} Z^{2}-i M_{B}^{A} \Upsilon_{A} \Theta^{B}=0 .
$$

(Again, we suppress the $L, R$ subscripts on fields.) Note that for $M_{B}^{A} \neq 0$, the left-moving gauge field $\widetilde{B}_{\overline{\mathfrak{z}}}$ and the right-moving gauge field $\widetilde{B}_{\mathfrak{z}}$ have to be related by complex conjugation, because the left-moving gauge transformation by itself is anomalous, with an anomaly proportional to $M_{B}^{A} M^{B}{ }_{A}$.

According to the previous discussion, and in particular (4.43), the mass deformation corresponds to an open string vertex operator and therefore should manifest itself as a change in the worldsheet boundary conditions. How can we convert (4.45) to a change of boundary conditions?

Let us first recall, on the classical level, what the equations of motion of the Berkovits action are. The charged fields have equations of motion

$$
\nabla_{\overline{\mathfrak{z}}} Z_{L}^{i}=0, \quad \nabla_{\overline{\mathfrak{z}}} Y_{L i}=0, \quad \nabla_{\mathfrak{z}} Z_{R i}=0, \quad \nabla_{\mathfrak{z}} Y_{R}^{i}=0, \quad i=1, \ldots, 4,
$$

and similarly for $\Upsilon_{A}$ and $\Theta^{A}$. The gauge fields $A_{\mathfrak{z}}, A_{\overline{\mathfrak{z}}}$ and $\widetilde{B}_{\mathfrak{z}}, \widetilde{B}_{\overline{\mathfrak{z}}}$ do not have dynamical equations of motion, and they can be arbitrary. The equations of motion (4.46) then imply that gauge invariant combinations of left-moving fields are holomorphic, and gauge invariant combinations of right-moving fields are anti-holomorphic. This (anti-)analyticity and the constraints

$$
\sum_{i=1}^{4} Y_{L i} Z_{L}^{i}+\Upsilon_{L A} \Theta_{L}^{A}=0, \quad \sum_{i=1}^{4} Y_{R}^{i} Z_{R i}+\Upsilon_{R}^{A} \Theta_{R A}=0
$$


together with (4.45), are all the restrictions on gauge invariant combinations. For $M_{B}^{A}=0$, a full set of $\widetilde{B}$-gauge invariant combinations is given by (3.36)-(3.37). For $M^{A}{ }_{B} \neq 0$, we need to modify these formulas. We define the following field combinations,

$$
\begin{aligned}
& Z^{1}, \quad Z^{2}, \quad W:=Z^{1} Z^{4}+Z^{2} Z^{3}, \quad U:=\frac{Y_{4}}{Z^{1}}=\frac{Y_{3}}{Z^{2}}-\frac{i}{Z^{1} Z^{2}} M^{A}{ }_{B} \Upsilon_{A} \Theta^{B} \\
& \widetilde{Y}_{1}:=Y_{1}-U Z^{4}+i \frac{Z^{3}}{\left(Z^{1}\right)^{2}} M^{A}{ }_{B} \Upsilon_{A} \Theta^{B}, \quad \widetilde{Y}_{2}:=Y_{2}-U Z^{3}, \\
& \widetilde{\Theta}:=\exp \left(i \frac{Z^{3}}{Z^{1}} M\right) \Theta, \quad \widetilde{\Upsilon}:=\exp \left(-i \frac{Z^{3}}{Z^{1}} M\right) \Upsilon .
\end{aligned}
$$

They are invariant under the gauge transformations that correspond to $\widetilde{B}$, and using the constraint (4.47), we see that they satisfy

$$
0=\widetilde{Y}_{1} Z^{1}+\widetilde{Y}_{2} Z^{2}+\widetilde{\Upsilon}_{A} \widetilde{\Theta}^{A}+2 U W
$$

Thus, the equations of motion for $Z^{1}, Z^{2}, \widetilde{Y}_{1}, \widetilde{Y}_{2}, W, U, \widetilde{\Theta}, \widetilde{\Upsilon}$ are independent of the mass matrix $M_{B}^{A}$. The mass matrix must, therefore, enter into the boundary conditions.

Let us consider a worldsheet with the topology of a disk. It can be represented as the upper half plane $\operatorname{Im} \mathfrak{z}>0$. The boundary conditions at $\operatorname{Im} \mathfrak{z}=0$ are [14]

$$
Z_{L}^{i}=Z_{R i}^{*}, \quad Y_{L i}=\left(Y_{R}^{i}\right)^{*}, \quad \Theta_{L}^{A}=\Theta_{R A}^{*}, \quad \Upsilon_{L A}=\left(\Upsilon_{R}^{A}\right)^{*}
$$

The "doubling trick" (see, e.g., §2.6 of [46]) is a standard way of treating a conformal field theory on a disk. We extend the definition of the left-moving fields to the full $\mathfrak{z}$-plane by setting

$$
Z_{L}^{i}(\mathfrak{z}):=\left(Z_{R}^{i}(\overline{\mathfrak{z}})\right)^{*}, \quad Y_{L i}(\mathfrak{z})=\left(Y_{R}^{i}(\overline{\mathfrak{z}})\right)^{*}, \quad \Theta_{L}^{A}(\mathfrak{z})=\Theta_{R A}(\overline{\mathfrak{z}})^{*}, \quad \Upsilon_{L A}(\mathfrak{z})=\left(\Upsilon_{R}^{A}(\overline{\mathfrak{z}})\right)^{*}, \quad \operatorname{Im} \mathfrak{z}<0 .
$$

Including the point $\mathfrak{z}=\infty$, the fields are now defined on a sphere.

Now let us look at the fields from (4.48). They are well-defined only if $Z^{1} \neq 0$. When $M_{B}^{A}=0$, we can use the alternative definition $U=Y_{3} / Z^{2}$, which makes sense when $Z^{2} \neq 0$. Thus, when $M_{B}^{A}=0$, the fields from (4.48) make sense whenever either $Z^{1}$ or $Z^{2}$ is nonzero. But for $M_{B}^{A} \neq 0$ we have to use a different set of fields in patches of the worldsheet that contain zeroes of $Z^{1}$. Such an alternative set of fields is given by

$$
\begin{aligned}
& Z^{1}, \quad Z^{2}, \quad W:=Z^{1} Z^{4}+Z^{2} Z^{3}, \quad U:=\frac{Y_{3}}{Z^{2}}=\frac{Y_{4}}{Z^{1}}+\frac{i}{Z^{1} Z^{2}} M^{A}{ }_{B} \Upsilon_{A} \Theta^{B}, \\
& \widetilde{Y}_{1}:=Y_{1}-U Z^{4}, \quad \widetilde{Y}_{2}:=Y_{2}-U Z^{3}-i \frac{Z^{4}}{\left(Z^{2}\right)^{2}} M^{A}{ }_{B} \Upsilon_{A} \Theta^{B}, \\
& \widetilde{\Theta}:=\exp \left(-i \frac{Z^{4}}{Z^{2}} M\right) \Theta, \quad \widetilde{\Upsilon}:=\exp \left(i \frac{Z^{4}}{Z^{2}} M\right) \Upsilon .
\end{aligned}
$$


These fields were determined by the requirement of invariance under $\widetilde{B}$-gauge transformations and the requirement that (4.49) is satisfied.

We still need to specify which of the two sets of formulas, (4.48) or (4.52), to choose for the left-moving and the right-moving sectors. We do not have any compelling reason to prefer one choice over the other, but if we choose (4.52) for the right-movers and (4.48) for the left-movers we will soon see that we recover the mass operator (4.43). It is not clear to us why we cannot choose the same set of formulas, say (4.48), for both left-movers and right-movers, but we note that if we do that we need a certain condition, say $Z_{L}^{1} \neq 0$, to hold throughout the doubled worldsheet, and this could be too restrictive.

With (4.48) for the left-movers and (4.52) for the right-movers, we get the boundary conditions

$$
\begin{aligned}
& Z_{L}^{1}=Z_{R 1}^{*}, \quad Z_{L}^{2}=Z_{R 2}^{*}, \quad W_{L}=W_{R}^{*}, \quad U_{L}=U_{R}^{*}+\frac{i}{Z_{L}^{1} Z_{L}^{2}} M^{A}{ }_{B} \Upsilon_{L A} \Theta_{L}^{B}, \\
& \widetilde{Y}_{1 L}=\widetilde{Y}_{1 R}^{*}+\frac{i W_{L}}{\left(Z_{L}^{1}\right)^{2} Z_{L}^{2}} M^{A}{ }_{B} \Upsilon_{L A} \Theta_{L}^{B}, \quad \widetilde{Y}_{2 L}=\widetilde{Y}_{2 R}^{*}-\frac{i W_{L}}{Z_{L}^{1}\left(Z_{L}^{2}\right)^{2}} M^{A}{ }_{B} \Upsilon_{L A} \Theta_{L}^{B} \\
& \Theta_{L}^{A}=\exp \left(i \frac{W_{L}}{Z_{L}^{1} Z_{L}^{2}} M\right) \Theta_{R A}^{*}, \quad \Upsilon_{L A}=\exp \left(-i \frac{W_{L}}{Z_{L}^{1} Z_{L}^{2}} M\right)\left(\Upsilon_{R}^{A}\right)^{*}
\end{aligned}
$$

These boundary conditions can be succinctly described by adding an extra boundary term to the worldsheet action,

$$
\delta S=-\frac{i}{2} \int d \mathfrak{z} \frac{W_{L}}{Z_{L}^{1} Z_{L}^{2}} \Upsilon_{L A} M^{A}{ }_{B} \Theta_{L}^{B}+\frac{i}{2} \int d \overline{\mathfrak{z}} \frac{W_{R}}{Z_{R}^{1} Z_{R}^{2}} \Upsilon_{R}^{B} M^{A}{ }_{B} \Theta_{R A} .
$$

To first order in $M$, the full action is given by the bulk worldsheet action (3.30) plus the boundary action (4.54), supplemented with the boundary conditions

$$
Z_{L}^{1}=Z_{R 1}^{*}, \quad Z_{L}^{2}=Z_{R 2}^{*}, \quad W_{L}=W_{R}^{*}, \quad \Theta_{L}^{A}=\exp \left(i \frac{W_{L}}{Z_{L}^{1} Z_{L}^{2}} M\right) \Theta_{R A}^{*} .
$$

The remaining boundary conditions (4.53) follow (upto $O\left(M^{2}\right)$ corrections) by minimizing the action. To conclude, we note that the boundary term (4.54) is consistent with the mass operator (4.43) that was predicted from the B-model.

Similarly to (4.43), we can convert (4.13) to Berkovits's model. Using the fields from Table 2 , we get the open string vertex operator

$$
V_{(\mathbf{1 0})} \rightarrow \frac{1}{Z^{1} Z^{2}} M^{A B} \epsilon_{B C D E} \Upsilon_{A} \Theta^{C} \Theta^{D} \Theta^{E}
$$


The mass terms in $\overline{\mathbf{1 0}}$, which were difficult to identify in the B-model mini-twistor string theory, can be readily identified in Berkovits's model. Dimensionally reducing the operators $E_{A B}$ from [14, we get the open string vertex operator

$$
V_{(\overline{\mathbf{1 0}})} \rightarrow \frac{1}{Z^{1} Z^{2}} M_{A B} \Theta^{A} \partial \Theta^{B} .
$$

\subsection{Application of $\operatorname{Spin}(7)$ R-symmetry}

In $\$ 4.2$ and $\$ 4.5$ we found three kinds of $D=3$ mass operators. We found the operators (4.55D) in the $\mathbf{1 0}$ of $S U(4)$, the operators (4.56) in the $\overline{\mathbf{1 0}}$ of $S U(4)$, and the operators (4.43) in the 15 of $S U(4)$. The latter were the easiest to analyze, since they could be derived by a twisted dimensional reduction. However, all these operators should be related by the Spin(7) R-symmetry that we discussed in 93.9 In this subsection we will apply the R-symmetry generators (3.44) to the operators (4.43). We will discover a surprise: the operators (4.43), (4.55) and (4.56) do not fit into an irreducible representation of $\operatorname{Spin}(7)$ !

Nevertheless, starting with the operators (4.56) (in the $\overline{\mathbf{1 0}}$ ), we can reconstruct a good $\operatorname{Spin}(7)$ multiplet of operators by successively applying the $\operatorname{Spin}(7)$ generators (3.44). These operators will be different from our previous results (4.43), (4.55). We can "distill" out of (4.43) the terms that do fall into the irreducible representation 35 and use them to complete the $\operatorname{Spin}(7)$ multiplet.

Let us now do this in detail. Set

$$
\Xi:=\frac{1}{Z^{1} Z^{2}} .
$$

The various mass terms that we found in equations (4.43), (4.55) and (4.56) are

$$
\begin{aligned}
& V_{(\overline{\mathbf{1 0}})} \rightarrow \Xi M_{A B} \Theta^{A} \partial \Theta^{B}, \\
& V_{(\mathbf{1 5 )}} \rightarrow \Xi W \Upsilon_{A} M_{B}^{A} \Theta^{B}, \\
& V_{(\mathbf{1 0})} \rightarrow \Xi M^{A B} \epsilon_{B C D E} \Upsilon_{A} \Theta^{C} \Theta^{D} \Theta^{E} .
\end{aligned}
$$

These terms are linear combinations of the vertex operators

$$
\begin{aligned}
& \mathcal{V}^{A B}:=\Xi \Theta^{(A} \partial \Theta^{B)}, \quad \tilde{\mathcal{V}}_{B}^{A}:=\Xi W\left(\Upsilon_{B} \Theta^{A}-\frac{1}{4} \delta_{B}^{A} \Upsilon_{C} \Theta^{C}\right), \\
& \tilde{\mathcal{V}}_{A B}:=\Xi \epsilon_{C D E(B} \Upsilon_{A)} \Theta^{C} \Theta^{D} \Theta^{E} .
\end{aligned}
$$

(Here and in the equations below, all operators are understood to be normal ordered.) As we will see below, some of these vertex operators are not quite what we are looking for, and the tilde 
over $\mathcal{V}$ will remind us that they are about to be modified. We will denote the modified vertex operators by $\mathcal{V}^{A}{ }_{B}$ and $\mathcal{V}_{A B}$. These vertex operators should form an irreducible representation of Spin(7), isomorphic to 35 . We can therefore write down immediately their expected commutation relations with the $\operatorname{Spin}(7)$ generators (3.42):

$$
\begin{aligned}
& {\left[T_{B}^{A}, \mathcal{V}_{C D}\right]=\delta_{C}^{A} \mathcal{V}_{B D}+\delta_{D}^{A} \mathcal{V}_{B C}} \\
& {\left[T^{A}{ }_{B}, \mathcal{V}^{C D}\right]=-\delta_{B}^{C} \mathcal{V}^{A D}-\delta_{B}^{D} \mathcal{V}^{A C}} \\
& {\left[T^{A}{ }_{B}, \mathcal{V}^{C}{ }_{D}\right]=\delta_{D}^{A} \mathcal{V}^{C}{ }_{B}-\delta_{B}^{C} \mathcal{V}^{A}{ }_{D}}
\end{aligned}
$$

and

$$
\begin{aligned}
& {\left[T^{A B}, \mathcal{V}_{C D}\right]=\delta_{C}^{A} \mathcal{V}^{B}{ }_{D}-\delta_{C}^{B} \mathcal{V}^{A}{ }_{D}+\delta_{D}^{A} \mathcal{V}^{B}{ }_{C}-\delta_{D}^{B} \mathcal{V}^{A}{ }_{C}} \\
& {\left[T^{A B}, \mathcal{V}^{C D}\right]=\epsilon^{A B C E} \mathcal{V}^{D}{ }_{E}+\epsilon^{A B D E} \mathcal{V}^{C}{ }_{E}} \\
& {\left[T^{A B}, \mathcal{V}^{C}{ }_{D}\right]=\epsilon^{A B C E} \mathcal{V}_{E D}+\delta_{D}^{A} \mathcal{V}^{B C}-\delta_{D}^{B} \mathcal{V}^{A C}}
\end{aligned}
$$

Thus, we can calculate the operators $\mathcal{V}^{A}{ }_{B}$ from the commutation relations of $T^{A B}$ with $\mathcal{V}^{C D}$.

We can read off these commutation relations from the coefficient of the simple pole in the OPE of $\mathcal{J}^{A B}$ and $\mathcal{V}^{C D}$. The result is

$$
\begin{aligned}
{\left[T^{A B}, \mathcal{V}^{C D}\right]=} & \frac{1}{2} \epsilon^{A B C E} \Xi\left(U^{-1} \partial \Upsilon_{E} \Theta^{D}+\partial U^{-1} \Upsilon_{E} \Theta^{D}-U^{-1} \Upsilon_{E} \partial \Theta^{D}\right) \\
& +\frac{1}{2} \epsilon^{A B D E} \Xi\left(U^{-1} \partial \Upsilon_{E} \Theta^{C}+\partial U^{-1} \Upsilon_{E} \Theta^{C}-U^{-1} \Upsilon_{E} \partial \Theta^{C}\right)
\end{aligned}
$$

A comparison with (4.60) teaches us that

$$
\mathcal{V}^{A}{ }_{B}=\frac{1}{2} \Xi\left(U^{-1} \partial \Upsilon_{B} \Theta^{A}+\partial U^{-1} \Upsilon_{B} \Theta^{A}-U^{-1} \Upsilon_{B} \partial \Theta^{A}\right)-\frac{1}{8} \delta_{B}^{A} \Xi\left(U^{-1} \partial \Upsilon_{C} \Theta^{C}+\partial U^{-1} \Upsilon_{C} \Theta^{C}-U^{-1} \Upsilon_{C} \partial \Theta^{C}\right)
$$

We have subtracted the trace to make $\mathcal{V}_{B}^{A}$ traceless, as is required of the irreducible representation 15.

Next, we calculate the commutation relations between the newly found $\mathcal{V}^{A}{ }_{B}$ and the $\operatorname{Spin}(7)$ generators $T^{A B}$. A long but straightforward calculation gives

$$
\left[T^{A B}, \mathcal{V}^{C}{ }_{D}\right]=-\delta_{D}^{[A} \Xi \partial \Theta^{B]} \Theta^{C}+\delta_{D}^{[A} \Xi \Theta^{B]} \partial \Theta^{C}-\epsilon^{A B C E} \Xi U^{-2} \partial \Upsilon_{(E} \Upsilon_{D)}
$$

A comparison with (4.60) therefore teaches us that

$$
\mathcal{V}_{A B}=U^{-2} \Xi \Upsilon_{(A} \partial \Upsilon_{B)}
$$

where $\Xi$ was defined in (4.57), and using the bosonized fields from $\$ 3.10$, we have defined

$$
U^{-2}:=-e^{-2 \phi} \xi \partial \xi
$$


This definition is natural, given the definition of $U^{-1}$ in (3.46) and the following OPE

$$
U^{-1}(\mathfrak{z}) U^{-1}(0)=-e^{-2 \phi(0)} \xi(0) \partial \xi(0)+O(\mathfrak{z})
$$

We can now write down our final result for the three types of mass operators:

$$
\begin{aligned}
\mathcal{V}^{A B}= & \Xi \Theta^{(A} \partial \Theta^{B)}, \\
\mathcal{V}_{A B}= & \Xi U^{-2} \Upsilon_{(A} \partial \Upsilon_{B)}, \\
\mathcal{V}_{B}^{A}= & \frac{1}{2} \Xi\left(U^{-1} \partial \Upsilon_{B} \Theta^{A}-U^{-1} \Upsilon_{B} \partial \Theta^{A}+\partial U^{-1} \Upsilon_{B} \Theta^{A}\right) \\
& -\frac{1}{8} \Xi \delta_{B}^{A}\left(U^{-1} \partial \Upsilon_{C} \Theta^{C}-U^{-1} \Upsilon_{C} \partial \Theta^{C}+\partial U^{-1} \Upsilon_{C} \Theta^{C}\right) .
\end{aligned}
$$

We have checked that these operators now satisfy all the relations (4.59)-(44.60) and hence constitute an irreducible representation of the R-symmetry group $\operatorname{Spin}(7)$ that is isomorphic to 35.

Our results are summarized in Table 3. The operator (4.64) agrees with the previous result (4.56), but the other operators (4.65)-(4.66) do not agree with the operators (4.55) and (4.43) that we found in 4.5 . The reason for this apparent discrepancy is not completely clear to us, but we expect the difference between (4.43) to (4.66) to decouple from physical amplitudes. As for the relation between (4.55) and (4.65), we suspect that these operators are in a different "picture," as we will now explain.

In 24] it was explained that vertex operators in Berkovits's model come in different "pictures." A disk amplitude with $(d+1)$ negative-helicity gluons and $(n-d-1)$ positive-helicity gluons requires an insertion of $d$ "instanton-changing operators," in addition to the $n$ vertex operators that correspond to the physical states. These instanton-changing operators are analogous to the picture-changing operators of superstring theory. They contain $\delta$-functions of the $Y_{I}$-fields, and are necessary to absorb zero-modes of those fields. Similarly to superstring theory, one can get rid of a picture-changing operator by absorbing it in a physical vertex operator, thereby changing the "picture" of that vertex operator. This observation played a crucial role in identifying parity symmetry 24 .

Let us now look at the zero-modes of $U$. Using the bosonization formulas (3.45), we can define the $U$-picture of an operator to be its $\phi$-momentum, so that an operator that contains an exponent $\exp (p \phi)$ will be in the $p$-picture. It follows from (3.44) that when $T^{A B}$ acts on an operator in the $p$-picture, it produces a sum of operators in the $(p-1)$-picture and $(p+1)$-picture. 


\begin{tabular}{|l|c|c|c|}
\hline \hline Operator & $M_{A B} \mathcal{V}^{A B}$ & $M^{A}{ }_{B} \mathcal{V}^{B}{ }_{A}$ & $M^{A B} \mathcal{V}_{A B}$ \\
\hline$S U(4)$ representation & $\overline{\mathbf{1 0}}$ & $\mathbf{1 5}$ & $\mathbf{1 0}$ \\
\hline Fermion coupling & $M_{A B} \chi^{A} \chi^{B}$ & $M^{A}{ }_{B} \chi^{A} \chi_{B}$ & $M^{A B} \chi_{A} \chi_{B}$ \\
\hline Conformal SUGRA field & $E_{A B}$ & $V_{4}{ }_{B}{ }_{B}$ & $\bar{E}^{A B}$ \\
\hline B-model deformation: $\delta \theta^{A}=$ & - & $z^{-1} M^{A}{ }_{B} w \theta^{B}$ & $\frac{1}{6} z^{-1} M^{A B} \epsilon_{B C D E} \theta^{C} \theta^{D} \theta^{E}$ \\
\hline Berkovits model & $\Xi \Theta^{(A} \partial \Theta^{B)}$ & $\frac{1}{2} \Xi U^{-1} \partial \Upsilon_{B} \Theta^{A}+\cdots$ & $\Xi U^{-2} \Upsilon_{(A} \partial \Upsilon_{B)}$ \\
\hline \hline
\end{tabular}

Table 3: The three types of mass operators with their $S U(4)$ irreducible representations, their coupling to the fermions, the corresponding conformal supergravity fields, the B-model complex structure deformations $\left(\delta \theta^{A}\right.$ is the extra term in the change of holomorphic variables from the patch $z \neq 0$ to the patch $z \neq \infty$ ) and the Berkovits-model operators from (4.64)-(4.66). The operator $\Xi \equiv 1 / Z^{1} Z^{2}$ was defined in (4.57).

To see why picture-changing is necessary, recall our comment at the end of \$3.1. There we saw that the notion of helicity in $D=3$ is not invariant under a general R-symmetry transformation. For example, we saw that a positive-helicity gluon satisfies $\Phi^{7}=i \Phi^{8}$, but one can find an infinitesimal R-symmetry transformation that acts as $\delta \Phi^{7}=\epsilon \Phi^{6}$, say. After this transformation, the gluon state will acquire a 0-helicity component. But replacing a $(+1)$-helicity state with a 0-helicity state in a scattering amplitude requires, according to [24], an extra instanton-changing operator.

Now take a positive-helicity gluon vertex operator in Berkovits's model. In $D=3$ it is of the form $\Phi\left(Z^{1}, Z^{2}, W\right) J_{C}$, where $J_{C}$ is a holomorphic current from the chiral-current algebra component of the worldsheet theory, and $\Phi$ is a meromorphic function. Acting with $T^{A B}$ gives

$$
\left[T^{A B}, \Phi\right]=\frac{\partial \Phi}{\partial W} \Theta^{A} \Theta^{B}+\frac{1}{2} \widetilde{\Phi}\left(Z^{1}, Z^{2}, U\right) \epsilon^{A B C D} \Upsilon_{C} \Upsilon_{D}
$$

where $\widetilde{\Phi}$ is the residue of the simple pole in the OPE of $\Phi$ with $U^{-1}$ and can be calculated from formula (A.3). The first term on the righthand side of (4.67) describes the above-mentioned 0-helicity state. The second term, however, is in a different $U$-picture and would formally correspond to a $(+2)$-helicity state. Since no such term exists, it must decouple from physical amplitudes. Similarly, the mass operators (4.65) and (4.55) are obviously in a different picture, since one contains $U^{-2}$ while the other does not.

We conclude this subsection by demonstrating explicitly that the operators $\tilde{\mathcal{V}}_{B}^{A}$ are not in the irreducible representation 35, as we claimed at the beginning of this subsection. If they were, we could complete them to a Spin(7) multiplet using the commutation relations (4.60), just like 
we did above with $\mathcal{V}^{A B}$. But an explicit computation gives

$$
\begin{aligned}
{\left[T^{A B}, \tilde{\mathcal{V}}_{C}^{D}\right]=} & 2 \delta_{C}^{[A} \Xi \partial \Theta^{B]} \Theta^{D}+2 \Xi \partial \phi \delta_{C}^{[A} \Theta^{B]} \Theta^{D} \\
& +\epsilon^{A B D E} \Xi U^{-2} \partial \Upsilon_{E} \Upsilon_{C}-\epsilon^{A B D E} \Xi U^{-2} \partial \phi \Upsilon_{E} \Upsilon_{C} \\
& +\Xi \Theta^{A} \Theta^{B} \Theta^{D} \Upsilon_{C}+\frac{1}{2} \epsilon^{A B E F} \Xi U^{-2} \Upsilon_{E} \Upsilon_{F} \Upsilon_{C} \Theta^{D}
\end{aligned}
$$

Here $\partial \phi=-: U W:$ is a bosonized current. If (4.60) were satisfied, we could set

$$
\begin{aligned}
\tilde{\mathcal{V}}_{A B}= & \frac{1}{6} \epsilon_{C D E A}\left[T^{C D}, \tilde{\mathcal{V}}_{B}^{E}\right] \\
= & U^{-2} \Xi \partial \Upsilon_{(A} \Upsilon_{B)}+\frac{1}{12} \epsilon_{C D E(A} \Theta^{C} \Theta^{D} \Theta^{E} \Upsilon_{B)} \\
& +\frac{1}{2} U^{-2} \Xi \partial\left(\Upsilon_{A} \Upsilon_{B}\right)-\Xi \Upsilon_{A} \Upsilon_{B} U^{-2} \partial \phi-\frac{1}{3} \Xi \epsilon_{A B C D} \partial \Theta^{C} \Theta^{D}-\frac{1}{3} \Xi \epsilon_{A B C D} \partial \phi \Theta^{C} \Theta^{D} \\
& +\frac{1}{12} \epsilon_{C D E[A} \Xi \Theta^{C} \Theta^{D} \Theta^{E} \Upsilon_{B]}+\frac{1}{3} \Xi U^{-2} \Upsilon_{A} \Upsilon_{B} \Upsilon_{C} \Theta^{C} .
\end{aligned}
$$

But $\tilde{\mathcal{V}}_{A B}$ has to be symmetric in the indices $A, B$, and the last two lines of (4.69) are antisymmetric in $A, B$. This means that $\tilde{\mathcal{V}}_{A B}$ is a mixture of the $S U(4)$ irreducible representations 10 and 15. It suggests that our starting point, (4.58) for $\tilde{\mathcal{V}}_{B}^{A}$, is a mixture of the irreducible $\operatorname{Spin}(7)$ representations 35 and the adjoint $\mathbf{2 1}$. One can similarly check that

$$
\begin{aligned}
\tilde{\mathcal{V}}^{B C}= & \frac{1}{3}\left[T^{A B}, \tilde{\mathcal{V}}_{A}^{C}\right] \\
= & \Xi \partial \Theta^{(B} \Theta^{C)}+\frac{1}{6} \epsilon^{C D E(B} \Xi U^{-2} \Upsilon_{C} \Upsilon_{D} \Upsilon_{E} \Theta^{C)} \\
& -\frac{1}{6} \epsilon^{B C D E} \Xi U^{-2} \partial\left(\Upsilon_{D} \Upsilon_{E}\right)+\frac{1}{3} \epsilon^{B C D E} \Xi U^{-2} \partial \phi \Upsilon_{D} \Upsilon_{E}-\frac{1}{3} \Xi \Theta^{B} \Theta^{C} \Upsilon_{A} \Theta^{A} \\
& +\frac{1}{6} \epsilon^{D E F[B} \Xi U^{-2} \Upsilon_{D} \Upsilon_{E} \Upsilon_{F} \Theta^{C]}+\frac{1}{2} \Xi \partial\left(\Theta^{B} \Theta^{C}\right)+\Xi \partial \phi \Theta^{B} \Theta^{C}
\end{aligned}
$$

This is again not symmetric in $A, B$ and thus contains a mixture of the $S U(4)$ irreducible representations $\overline{\mathbf{1 0}}$ and $\mathbf{6}$.

\subsection{The singlet mass term}

The last remaining mass term in (4.1) is the singlet 1. A naive guess for the corresponding Bmodel operator is to set $M$ in (4.6) to be proportional to the identity matrix. This means that we cannot obtain (4.6) by dimensional reduction with an $S U(4)$ twist, because the identity matrix is not traceless. An even bigger problem is that if $M$ is not traceless, the complex structure deformation to which (4.6) corresponds does not preserve the holomorphic volume form $\Omega$ of $\mathbb{C P}^{3 \mid 4}$. Indeed, unless $M$ is traceless,

$$
\Omega=\frac{1}{4 !^{2}} \epsilon_{I J K L} Z^{I} d Z^{J} \wedge d Z^{L} \wedge d Z^{L} \epsilon_{A B C D} d \psi^{A} d \psi^{B} d \psi^{C} d \psi^{D}
$$


is not preserved by (4.3). We do not know how to turn on singlet mass terms. (See also the related discussion after equation (2.11) in $§ 2.2$ of [14] regarding complex structure deformations that do not preserve $\Omega$.)

\subsection{Comments on the decoupling limit $M \rightarrow \infty$.}

The discussion in the previous subsections was concerned mainly with infinitesimal mass terms. To first order, these mass terms are related to deformations of the B-model action by closed string vertex operators. Our analysis in $\$ 4.1$ however, allows us to "integrate" the infinitesimal mass deformations and describe finite mass terms. Specifically, (4.3) describes a super complex structure deformation of $\mathbb{C P}^{3 \mid 4}$ that preserves the holomorphic volume super-form for any traceless $M$.

Let $m_{1}, \ldots, m_{4}$ be the eigenvalues of $M$, and set $m_{i}=c_{i} m$ for some constants $c_{i}$. For generic $c_{i}$, the limit $m \rightarrow \infty$ is quite interesting from the physical perspective. If all $c_{i}$ and all $c_{i}+c_{j}$ are nonzero, all fermions and six out of the seven scalars get a large bare mass and decouple. We can preserve $D=3, N=2$ supersymmetry if we keep $c_{1}=0$. In this case, one scalar and two $D=3$ gluinos remain with zero bare mass. We can also preserve $D=3, N=4$ supersymmetry if we keep $c_{1}=c_{2}=0$. In this case, three scalars and four gluinos remain with zero bare mass.

Let us now comment on the $m \rightarrow \infty$ limit from the perspective of Berkovits's open twistor string theory. The mass appears only in the boundary term (4.54). In the limit $m \rightarrow \infty$, the boundary term (4.54) becomes very big. This suggests that the bulk action of $\Upsilon$ and $\Theta$ can be neglected. We end up with a worldsheet theory that has bulk modes $Z^{1}, Z^{2}, W$ and their conjugates $Y_{1}, Y_{2}, U$. The $\Upsilon$ and $\Theta$ fields live only on the boundary and couple to the bulk fields via the boundary action (4.54). This state of affairs is somewhat reminiscent of the SeibergWitten limit of large NS-NS 2-form $B$ field that leads to noncommutative geometry [49] [50] [51]. (A connection between deformations of twistor string theory and noncommutative geometry, and in particular its extension to superspace [52] was also suggested in [30].) This limit in the present context will be studied elsewhere [53].

\section{Conclusions and discussion}

We reviewed Hithcin's construction of mini-twistor space, which relates $D=3$ mini-twistor space to $D=4$ twistor space by dimensional reduction. The key point is that the 3 (complex)- 
dimensional $D=4$ twistor space $\mathbb{C P}^{3} \backslash \mathbb{C P}^{1}$ can be written as a fiber bundle with the 2 (complex)dimensional $D=3$ twistor space $T \mathbb{C P}^{1}$ as the base, and the fiber is $\mathbb{C}$. The structure group is the additive translation group $\sim \mathbb{C}$. We used this fibration to relate tree-level twistor amplitudes of $D=3$ Yang-Mills theory to $D=4$ amplitudes. We calculated $D=3$ tree-level amplitudes from the $D=4$ ones by integrating over the $\mathbb{C}$ fibers of the above fibration. This immediately implies that Witten's observations [4] regarding scattering amplitudes and holomorphic curves in twistor space are valid in $D=3$, at least at tree-level. In $D=3$ there is a known relation between holomorphic curves on mini-twistor space and holomorphic curves in complexified Minkowski space. We used this relation to give a direct physical interpretation to the holomorphic curves in mini-twistor space. At one-loop level and higher, the $D=3$ and $D=4$ amplitudes are not directly related by dimensional reduction, and whether or not the conjectures of [4] extend to $D=3$ remains to be seen. For developments regarding loop amplitudes in $D=4$ see 63, 65, 66, 67, 68, 69, 70].

Our main new results are related to deformations of the $N=8$ supersymmetric $D=3$ YangMills theory by mass terms. We proposed a variant of the topological B-model that describes massive $D=3$ Yang-Mills theory. This model can describe 15 out of the 35 different possible mass terms. These mass-deformed $D=3$ theories can be obtained by twisting the dimensional reduction of massless $D=4$ theories. As for the other mass terms, we only discussed infinitesimal deformations and conjectured that these deformations correspond to VEVs of certain conformal supergravity fields. In this paper, we gave circumstantial evidence in support of the previous statement. It would be very interesting to show this convincingly by examining the three point functions of two supergravity fields and one gauge field operator. This should be possible by performing a computation similar to the one in [14] [see equation (5.5) of that paper], where the correlation functions of a conformal supergravity vertex operator with an arbitrary number of Yang-Mills vertex operators were calculated in Berkovits's model. We hope to report on this in another paper [53].

In $D=3$ we constructed the $\operatorname{Spin}(7)$ R-symmetry currents that transform one type of infinitesimal mass term to another. This allowed us to construct vertex operators for all 35 mass terms in Berkovits's model. We encountered a surprise when we tried to compare the vertex operators in Berkovits's model with the topological B-model. In Berkovits's model we found that the 35 mass operators do not quite fit into an irreducible representation of the R-symmetry group $\operatorname{Spin}(7)$. We suggested a different set of operators that do fit into an R-symmetry multiplet. 
It would be interesting to understand the meaning of this in more detail.

Our results provide a way to break the supersymmetry from $N=8$ to $N=4,2,1,0$ in $D=3$ by arbitrary mass terms, in mini-twistor string theory. One direction for further research is to analyze the limit of infinite mass. In this limit we should obtain pure $N=4,2,1$ Yang-Mills theories, and also $N=0$ with a scalar. Our results also suggest a way to break $N=4$ in $D=4$ by infinitesimal mass terms. It would be interesting to try to "integrate" these infinitesimal deformations to get large mass terms. Understanding R-symmetry better may enable us to convert a non-infinitesimal mass term in the representation 15 [described by (4.3) in the B-model or (4.45) in Berkovits's model] into a mass term in the $\mathbf{1 0}+\overline{\mathbf{1 0}}$, and then it might be possible to lift it to $D=4$. Alternatively, a better understanding of the nonperturbative topological Bmodel, perhaps along the lines suggested recently in 39 [38 [40, may shed light on how to turn on non-infinitesimal VEVs for the closed string modes $E_{A B}$ and $\bar{E}^{A B}$ simultaneously.

Another possible direction for further research is to explore the mirror manifold of mini-twistor space. The same techniques of [54], where the mirror manifold of the twistor space $\mathbb{C P}^{3 \mid 4}$ has been constructed, can be applied to mini-twistor space. It was shown in [54] that the mirror of $\mathbb{C P}^{3 \mid 4}$ is a quadric in $\mathbb{C P}^{3 \mid 3} \times \mathbb{C P}^{3 \mid 3}$. One might then be tempted to examine the dimensionally reduced version of the argument in [72, where the authors use S-duality in conjunction with mirror symmetry to explain why the amplitudes of $\mathcal{N}=4$ Yang-Mills are supported on holomorphic curves. Moreover, certain symmetries (e.g. parity) might be more manifest in the mirror. One would expect to lose some of the manifest $S U(4 \mid 4)$ generators.

\section{Acknowledgments}

It is a pleasure to thank Mina Aganagic, Korkut Bardakci, Itzhak Bars, Iosif Bena, Eric Gimon, Nick Halmagyi, Hitoshi Murayama, Anthony Ndirango, Eliezer Rabinovici and Radu Tatar for helpful discussions. We also wish to thank Alexander Popov, Christian Sämann and Martin Wolf for comments on the first version of our paper. This work was supported in part by the Director, Office of Science, Office of High Energy and Nuclear Physics, of the U.S. Department of Energy under Contract DE-AC03-76SF00098, and in part by the NSF under grant PHY0098840. 


\section{A Dealing with inverses of fields}

The commuting worldsheet fields of Berkovits's model, $\left(Y_{1}, Z^{1}\right),\left(Y_{2}, Z^{2}\right)$ and $(U, W)$, have the same OPEs as the superconformal ghosts $(\beta, \gamma)$ in superstring theory. Based on this, we bosonized the fields $(U, W)$ in $\$ 3.10$. Actually, we will need to bosonize the three pairs $\left(Y_{1}, Z^{1}\right),\left(Y_{2}, Z^{2}\right)$ and $(U, W)$, simultaneously. To ensure that fields from different pairs are commuting (rather than anti-commuting) it is more convenient to use a bosonization scheme with no anti-commuting fields. Recall that any $(\beta, \gamma)$ pair of worldsheet fields with OPE

$$
\beta(\mathfrak{z}) \gamma(0) \sim-\frac{1}{\mathfrak{z}}
$$

can be bosonized in one of two ways:

$$
\text { Either }\left\{(\mathrm{i}) \quad \beta=e^{-\phi+\chi} \partial \chi, \quad \gamma=e^{\phi-\chi}\right\} \quad \text { or } \quad\left\{(\mathrm{ii}) \quad \beta=e^{-\phi+\chi}, \quad \gamma=e^{\phi-\chi} \partial \chi\right\} \text {, }
$$

where $\chi(\mathfrak{z})$ and $\phi(\mathfrak{z})$ are chiral bosons. The current in both cases is

$$
\beta \gamma=\partial \phi
$$

(See for example $\S 10.4$ of [46].)

At the outset, it seems that for each pair of fields $\left(Y_{i}, Z^{i}\right)$ or $(U, W)$, we can choose either the bosonization scheme (i) or (ii). How can we decide which scheme to choose?

Before we pick the bosonization scheme, we have to decide which of the $\beta, \gamma$ fields is to have an inverse. If it is $\gamma$, we should pick scheme (i), because we could then define

$$
\gamma^{-1}:=e^{-\phi+\chi} \quad \text { in bosonization scheme (i). }
$$

If it is $\beta$, we should pick scheme (ii) and set

$$
\beta^{-1}:=e^{\phi-\chi} \quad \text { in bosonization scheme (ii). }
$$

The field $\gamma$ does not have an inverse in scheme (i), and $\beta$ does not have an inverse in scheme (ii).

For the application to mini-twistor space we would like $Z^{1}, Z^{2}$ and $U$ to have inverses. The inverse of $U$ was needed in (3.44) for the $\operatorname{Spin}(7)$ R-symmetry current. The inverses $\left(Z^{1}\right)^{-1}$ and $\left(Z^{2}\right)^{-1}$ were needed for $\Xi$ [defined in (4.57)] and for all the mass operators that contained $\Xi$. The inverses of the other fields, $Y_{1}^{-1}, Y_{2}^{-1}, W^{-1}$ were never needed. 
More basically, if we allow $\left(Z^{i}\right)^{-1}(i=1,2)$ it means that we are restricting to the patch of mini-twistor space where $Z^{i} \neq 0$. If we proscribe $W^{-1}$ it means that we are including points where $w=0$. The discussion above compels us to choose the following bosonization scheme:

$$
\begin{aligned}
Y_{i} & =e^{-\phi_{i}+\chi_{i}} \partial \chi_{i}, \quad Z^{i}=e^{\phi_{i}-\chi_{i}}, \quad\left(Z^{i}\right)^{-1}=e^{-\phi_{i}+\chi_{i}}, \quad(i=1,2), \\
U & =e^{-\phi_{3}+\chi_{3}}, \quad W=e^{\phi_{3}-\chi_{3}} \partial \chi_{3}, \quad U^{-1}=e^{\phi_{3}-\chi_{3}} \\
j & =Y_{1} Z^{1}+Y_{2} Z^{2}+2 U W=\partial \phi_{1}+\partial \phi_{2}+2 \partial \phi_{3} .
\end{aligned}
$$

We conclude with a few useful OPEs:

$$
\begin{aligned}
U(\mathfrak{z}) U^{-1}(0) & \sim 1+\mathfrak{z}[\partial \phi(0)+\eta(0) \xi(0)]+O\left(\mathfrak{z}^{2}\right), \\
U(\mathfrak{z}) W(0) & \sim-\frac{1}{\mathfrak{z}}-\partial \phi(0)+O(\mathfrak{z}), \\
U^{-1}(\mathfrak{z}) W(0) & \sim \frac{1}{\mathfrak{z}} U^{-2}(0)-U^{-2}(0) \partial \phi(0)+O(\mathfrak{z}), \\
U^{-1}(\mathfrak{z}) U^{-1}(0) & \sim U^{-2}(0)+\frac{1}{2} \mathfrak{z} \partial U^{-2}+O(\mathfrak{z})^{2} \\
U^{-1}(\mathfrak{z}) U^{-2}(0) & \sim U^{-3}(0)+O(\mathfrak{z}), \\
U(\mathfrak{z}) \partial U^{-1}(0) & \sim-\partial \phi(0)-\eta(0) \xi(0)+O(\mathfrak{z}) . \\
U(\mathfrak{z}) U^{-2}(\mathfrak{z}) & \sim U(0)-\mathfrak{z} \partial\left(U^{-1}(0)\right)+\mathfrak{z}^{2} X(0)+O(\mathfrak{z})^{3}, \\
U^{-1}(\mathfrak{z}) \partial U^{-1}(0) & \sim \frac{1}{2} \partial U^{-2}(0)+O(\mathfrak{z})
\end{aligned}
$$

where we defined the operators

$$
X:=e^{-\phi}\left(\eta(\partial \xi) \xi-\partial \phi \partial \xi+\frac{1}{2}\left((\partial \phi)^{2}+\partial^{2} \phi\right) \xi\right)
$$

and

$$
U^{-2}:=e^{-2 \phi}(\partial \xi) \xi, \quad U^{-3}:=\frac{1}{2} e^{-3 \phi} \partial^{2} \xi(\partial \xi) \xi
$$

(All products on the righthand side are assumed to be normal ordered.) Finally, we need the OPEs

$$
U^{-1}(\mathfrak{z}) W^{n}(0)=\frac{1}{\mathfrak{z}}(-1)^{n} n ! U^{-n-1}+O(1)
$$

\section{References}

[1] S. J. Parke and T. R. Taylor, "An Amplitude For N Gluon Scattering," Phys. Rev. Lett. 56, 2459 (1986). 
[2] F. A. Berends and W. T. Giele, "Recursive Calculations For Processes With N Gluons," Nucl. Phys. B 306, 759 (1988).

[3] Z. Bern, L. J. Dixon, and D. A. Kosower, "Progress in one-loop QCD computations," Ann. Rev. Nucl. Part. Sci. 46, 109-148 (1996). arXiv:hep-ph/9602280

[4] E. Witten, "Perturbative gauge theory as a string theory in twistor space," arXiv:hep-th/0312171.

[5] R. Penrose, "Twistor algebra," J. Math. Phys. 8, 345 (1967)

[6] S. Gukov, L. Motl and A. Neitzke, "Equivalence of twistor prescriptions for super YangMills," arXiv:hep-th/0404085

[7] J. M. Maldacena, "The large N limit of superconformal field theories and supergravity," Adv. Theor. Math. Phys. 2, 231 (1998), Int. J. Theor. Phys. 38, 1113 (1999) arXiv:hep-th/9711200.

[8] S. S. Gubser, I. R. Klebanov and A. M. Polyakov, "Gauge theory correlators from noncritical string theory," Phys. Lett. B 428, 105 (1998) arXiv:hep-th/9802109.

[9] E. Witten, "Anti-de Sitter space and holography," Adv. Theor. Math. Phys. 2, 253 (1998) arXiv:hep-th/9802150.

[10] N. J. Hitchin, "Monopoles And Geodesics," Commun. Math. Phys. 83 (1982) 579.

[11] P. Baird, "An Introduction to Twistors," http://www.math.jussieu.fr/ helein/encyclopaedia/baird-twistors.pdf

[12] I. Bars, "Twistor superstring in 2T-physics," arXiv:hep-th/0407239.

[13] N. Berkovits, "An alternative string theory in twistor space for $\mathrm{N}=4$ super-Yang-Mills," Phys. Rev. Lett. 93, 011601 (2004) arXiv:hep-th/0402045.

[14] N. Berkovits and E. Witten, "Conformal supergravity in twistor-string theory," JHEP 0408, 009 (2004) arXiv:hep-th/0406051

[15] The Wolfram functions site, http://functions.wolfram.com/05.07.07.0002.01. 
[16] W. Siegel, "Untwisting the twistor superstring," arXiv:hep-th/0404255.

[17] V. P. Nair, "Yang-Mills theory, 2+1 and 3+1 dimensions," arXiv:hep-th/0309061.

[18] S. Sethi, "Supermanifolds, rigid manifolds and mirror symmetry," Nucl. Phys. B 430, 31 (1994) arXiv:hep-th/9404186.

[19] A. D. Popov and C. Sämann, "On supertwistors, the Penrose-Ward transform and $N=4$ super Yang-Mills theory," arXiv:hep-th/0405123.

[20] A. D. Popov and M. Wolf, "Topological B-model on weighted projective spaces and self-dual models in four dimensions," JHEP 0409, 007 (2004) arXiv:hep-th/0406224.

[21] C. Sämann, "The topological B-model on fattened complex manifolds and subsectors of $N=4$ self-dual Yang-Mills theory," arXiv:hep-th/0410292.

[22] M. Wolf, "On hidden symmetries of a super gauge theory and twistor string theory," arXiv:hep-th/0412163.

[23] R. Roiban, M. Spradlin and A. Volovich, "A googly amplitude from the B-model in twistor space," JHEP 0404, 012 (2004) arXiv:hep-th/0402016.

[24] N. Berkovits and L. Motl, "Cubic twistorial string field theory," JHEP 0404, 056 (2004) arXiv:hep-th/0403187

[25] D. Montano and J. Sonnenschein, "Topological Strings," Nucl. Phys. B 313, 258 (1989).

[26] Y. K. Cheung, O. J. Ganor and M. Krogh, "On the twisted $(2,0)$ and little-string theories," Nucl. Phys. B 536, 175 (1998) arXiv:hep-th/9805045.

[27] J. Park and S. J. Rey, "Supertwistor orbifolds: Gauge theory amplitudes and topological strings," arXiv:hep-th/0411123.

[28] M. B. Halpern and C. Helfgott, "The general twisted open WZW string," arXiv:hep-th/0406003 M. B. Halpern and F. Wagner, "The general coset orbifold action," Int. J. Mod. Phys. A 18, 19 (2003) arXiv:hep-th/0205143.

[29] S. Giombi, M. Kulaxizi, R. Ricci, D. Robles-Llana, D. Trancanelli and K. Zoubos, "Orbifolding the twistor string," arXiv:hep-th/0411171. 
[30] M. Kulaxizi and K. Zoubos, "Marginal deformations of $N=4$ SYM from open / closed twistor strings," arXiv:hep-th/0410122.

[31] R. G. Leigh and M. J. Strassler, "Exactly marginal operators and duality in four-dimensional $N=1$ supersymmetric gauge theory," Nucl. Phys. B 447, 95 (1995) arXiv:hep-th/9503121.

[32] O. Aharony, B. Kol and S. Yankielowicz, "On exactly marginal deformations of $N=4$ SYM and type IIB supergravity on $A d S_{5} \times S^{5}$," JHEP 0206, 039 (2002) arXiv:hep-th/0205090.

[33] N. Dorey and T. J. Hollowood, "On the Coulomb branch of a marginal deformation of $\mathrm{N}=$ 4 SUSY Yang-Mills," arXiv:hep-th/0411163.

[34] E. Witten, "Mirror manifolds and topological field theory," arXiv:hep-th/9112056.

[35] M. Bershadsky, S. Cecotti, H. Ooguri and C. Vafa, "Kodaira-Spencer theory of gravity and exact results for quantum string amplitudes," Commun. Math. Phys. 165, 311 (1994) arXiv:hep-th/9309140

[36] C. h. Ahn, " $N=1$ conformal supergravity and twistor-string theory," JHEP 0410, 064 (2004) arXiv:hep-th/0409195.

[37] M. Movshev and A. Schwarz, "On maximally supersymmetric Yang-Mills theories," Nucl. Phys. B 681, 324 (2004) arXiv:hep-th/0311132.

[38] N. Nekrasov, H. Ooguri and C. Vafa, "S-duality and topological strings," JHEP 0410, 009 (2004) arXiv:hep-th/0403167.

[39] M. Aganagic, R. Dijkgraaf, A. Klemm, M. Marino and C. Vafa, "Topological strings and integrable hierarchies," arXiv:hep-th/0312085.

[40] R. Dijkgraaf, S. Gukov, A. Neitzke and C. Vafa, "Topological M-theory as unification of form theories of gravity," arXiv:hep-th/0411073.

[41] M. Aganagic, H. Ooguri, N. Saulina and C. Vafa, "Black holes, q-deformed 2d Yang-Mills, and non-perturbative topological strings," arXiv:hep-th/0411280.

[42] C. Hofman and W. K. Ma, "Deformations of topological open strings," JHEP 0101, 035 (2001) arXiv:hep-th/0006120. 
[43] C. I. Lazaroiu, "On the structure of open-closed topological field theory in two dimensions," Nucl. Phys. B 603, 497 (2001) arXiv:hep-th/0010269.

[44] C. Hofman, "On the open-closed B-model," JHEP 0311, 069 (2003) arXiv:hep-th/0204157.

[45] P. Griffiths and J. Harris, Principles of Algebraic Geometry, Wiley-Interscience Publication, 1994.

[46] J. Polchinski, "String theory. Vol. 1: An introduction to the bosonic string," "String theory. Vol. 2: Superstring theory and beyond," Cambridge 2003.

[47] D. Polyakov, "GL(1) Charged States in Twistor String Theory," arXiv:hep-th/0501219.

[48] N. Berkovits, "Calculation of Green-Schwarz superstring amplitudes using the $N=2$ twistor string formalism," Nucl. Phys. B 395, 77 (1993) arXiv:hep-th/9208035.

[49] A. Connes, M. R. Douglas and A. Schwarz, "Noncommutative geometry and matrix theory: Compactification on tori," JHEP 9802, 003 (1998) arXiv:hep-th/9711162.

[50] M. R. Douglas and C. M. Hull, "D-branes and the noncommutative torus," JHEP 9802, 008 (1998) arXiv:hep-th/9711165.

[51] N. Seiberg and E. Witten, "String theory and noncommutative geometry," JHEP 9909, 032 (1999) arXiv:hep-th/9908142.

[52] N. Seiberg, "Noncommutative superspace, $N=1 / 2$ supersymmetry, field theory and string theory," JHEP 0306, 010 (2003) arXiv:hep-th/0305248.

[53] Work in progress.

[54] M. Aganagic and C. Vafa, "Mirror symmetry and supermanifolds," arXiv:hep-th/0403192.

[55] F. Cachazo, P. Svrcek and E. Witten, "MHV vertices and tree amplitudes in gauge theory," JHEP 0409, 006 (2004) arXiv:hep-th/0403047.

[56] P. Baird and J.C. Wood, "Harmonic morphisms and conformal foliations by geodesics of three-dimensional space forms," J. Austral. Math. Soc.,123, (1998) 273-300.

[57] A. Sinkovics and E. Verlinde, "A six dimensional view on twistors," arXiv:hep-th/0410014. 
[58] E. Witten, "An Interpretation Of Classical Yang-Mills Theory," Phys. Lett. B 77, 394 (1978).

[59] K. Zoubos, "Holography and quaternionic Taub-NUT," JHEP 0212, 037 (2002) arXiv:hep-th/0209235.

[60] B. Y. Hou, B. Y. Hou, X. H. Wang, C. H. Xiong and R. H. Yue, "The affine ambitwistor space as the moduli space of SUYM in $A d S_{5} \times S^{5}$," arXiv:hep-th/0406251.

[61] M. F. Atiyah and N. Hitchin, The Geometry And Dynamics Of Magnetic Monopoles (Princeton University Press, 1988).

[62] N. J. Hitchin, A. Karlhede, U. Lindstrom and M. Rocek, "Hyperkähler Metrics And Supersymmetry," Commun. Math. Phys. 108, 535 (1987).

[63] Z. Bern, L. J. Dixon and D. A. Kosower, "All non-maximally-helicity-violating one-loop seven-gluon amplitudes in $\mathrm{N}=4$ super-Yang-Mills theory," arXiv:hep-th/0410224.

[64] Z. Bern, L. J. Dixon and D. A. Kosower, "All next-to-maximally helicity-violating one-loop gluon amplitudes in N = 4 super-Yang-Mills theory," arXiv:hep-th/0412210.

[65] F. Cachazo, P. Svrcek and E. Witten, "Twistor space structure of one-loop amplitudes in gauge theory," JHEP 0410, 074 (2004) arXiv:hep-th/0406177.

[66] F. Cachazo, "Holomorphic anomaly of unitarity cuts and one-loop gauge theory amplitudes," arXiv:hep-th/0410077.

[67] R. Britto, F. Cachazo and B. Feng, "Generalized unitarity and one-loop amplitudes in $N=4$ super-Yang-Mills," arXiv:hep-th/0412103.

[68] J. Bedford, A. Brandhuber, B. Spence and G. Travaglini, "A twistor approach to one-loop amplitudes in $N=1$ supersymmetric Yang-Mills theory," Nucl. Phys. B 706, 100 (2005) arXiv:hep-th/0410280

[69] Z. Bern, L. J. Dixon and D. A. Kosower, "On-Shell Recurrence Relations for One-Loop QCD Amplitudes," arXiv:hep-th/0501240 
[70] S. J. Bidder, N. E. J. Bjerrum-Bohr, D. C. Dunbar and W. B. Perkins, "One-loop gluon scattering amplitudes in theories with $N<4$ supersymmetries," arXiv:hep-th/0502028.

[71] E. Witten, "Parity invariance for strings in twistor space," arXiv:hep-th/0403199.

[72] A. Neitzke, and C. Vafa, " $N=2$ strings and the twistorial Calabi-Yau," arXiv:hep-th/0402128. 


\begin{tabular}{|c|c|c|}
\hline Symbol & Subsection & Meaning \\
\hline$a, b, \ldots$ & $\oint 3.1$ & Spin(7) spinor R-symmetry indices $(1 \ldots 8)$ \\
\hline$A, B, C, \ldots$ & $\$ 2.5$ & $S U(4)$ R-symmetry indices $(1 \ldots 4)$ \\
\hline$A_{\mu}$ & $\$ 3.1$ & spacetime gauge field (either $D=3$ or $D=4$ ) \\
\hline$A_{\mathfrak{z}}, A_{\overline{\mathfrak{z}}}$ & $\$ 3.8$ & worldsheet $G L(1)$ gauge field \\
\hline$\widetilde{B}_{\mathfrak{z}}, \widetilde{B}_{\overline{\mathfrak{z}}}$ & $\$ 3.8$ & worldsheet gauge field, used to gauge for translations \\
\hline$E_{A B}, \bar{E}^{A B}$ & $\$ 3.9$ & spacetime conformal supergravity fields from [14] \\
\hline$f, f^{\prime}$ & 4.5 & holomorphic vector field on mini-twistor space \\
\hline $\mathcal{F}$ & $\$ 3.6$ & focal curve \\
\hline$i, j, \ldots$ & $\$ 3.1$ & Euclidean spacetime indices $1, \ldots, 3$ or $1, \ldots, 4$ \\
\hline$I, \ldots$ & $\$ 3.1$ & $\operatorname{Spin}(7)$ vector index $1, \ldots, 7$ \\
\hline I & $\$ 3.10$ & a collective index for one of the eight $D=4$ Berkovits-model fields \\
\hline$J_{ \pm}, J_{1}$ & $\oint 2.4$ & $D=3$ rotation generators \\
\hline $\mathcal{L}$ & $\$ 3.1$ & spacetime Lagrangian \\
\hline$M_{B}^{A}, M_{A B}, M^{A B}$ & 94.1 & Mass matrices \\
\hline$P_{ \pm}, P_{1}$ & $\$ 2.4$ & $D=3$ translation generators \\
\hline$Q_{ \pm}, \bar{Q}_{ \pm}$ & $\$ 2.5$ & $D=3$ supersymmetry generators \\
\hline$T^{A B}, T_{B}^{A}$ & $\$ 3.9$ & $\operatorname{Spin}(7) \mathrm{R}$-symmetry generators \\
\hline $\mathcal{J}^{A B}, \mathcal{J}^{A}{ }_{B}$ & $\$ 3.10$ & $\operatorname{Spin}(7) \mathrm{R}$-symmetry currents \\
\hline $\mathfrak{t}$ & $\oint 2.6$ & $D=3$ mini-twistor \\
\hline $\mathbb{T}_{3}$ & $\$ 2.5$ & Mini-twistor space \\
\hline$U_{1}, U_{2}$ & $\$ 3.3$ & patches of mini-twistor space \\
\hline$U$ & $\$ 3.8$ & worldsheet Berkovits-model field dual to $W$ \\
\hline $\mathcal{V}^{A}{ }_{B}, \mathcal{V}^{A B}, \mathcal{V}_{A B}$ & 4.6 & Berkovits-model mass operators \\
\hline$\tilde{\mathcal{V}}_{B}^{A}, \tilde{\mathcal{V}}^{A B}, \tilde{\mathcal{V}}_{A B}$ & 4.6 & Berkovits-model mass operators (converted from B-model) \\
\hline$\tilde{W}, \tilde{W}$ & $\$ 3.7$ & B-model worldsheet field $(D=3)$ \\
\hline$W$ & $\$ 3.8$ & Berkovits-model worldsheet fields $(D=3)$ \\
\hline$w, \bar{w}$ & $\$ 2.2$ & coordinates on mini-twistor space (for the fiber of $T \mathbb{C P}^{1}$ ) \\
\hline$X^{i}$ & $\$ 4.4$ & worldsheet fields in $D=4$ B-model $(i=1, \ldots, 4)$ \\
\hline$x_{\alpha \dot{\alpha}}$ & $\$ 3.2$ & spacetime coordinate $(D=3$ or $D=4)$ \\
\hline$Y_{i}=$ & $\$ 3.8$ & Berkovits model dual field $(i=1,2$ in $D=3$ ) \\
\hline$\tilde{Z}, \bar{Z}$ & $\$ 3.7$ & B-model worldsheet fields $(D=3)$ \\
\hline$Z^{i}$ & $\$ 3.8$ & worldsheet fields in $D=3$ Berkovits model $(i=1,2)$ \\
\hline$z, \bar{z}$ & 22.2 & coordinate on mini-twistor space (for the base of $T \mathbb{C P}^{1}$ ) \\
\hline $\mathfrak{z}, \overline{\mathfrak{z}}$ & $\$ 3.8$ & worldsheet coordinate \\
\hline
\end{tabular}

Table 4: Various symbols used in the text (Roman letters). The "subsection" column is the place where the symbol first appeared with its given meaning. 


\begin{tabular}{|c|c|c|}
\hline Symbol & Subsection & Meaning \\
\hline$\alpha, \beta, \ldots$ & 92.7 & chiral spinor indices $(D=3$ or $D=4)$ \\
\hline$\dot{\alpha}, \dot{\beta}, \ldots$ & $\$ 3.1$ & dotted spinors $(D=4)$ \\
\hline$\Gamma^{\mu}$ & $\$ 2.7$ & $D=3$ Dirac matrices \\
\hline$\zeta_{A}$ & $\$ 4.4$ & holomorphic component function on twistor space \\
\hline$\zeta$ & 4.4 & arbitrary function of $\lambda, \hat{\lambda}$ \\
\hline$\widehat{\zeta}$ & 4.4 & twistor transform of $\zeta$ \\
\hline$\eta$ & $\$ 3.2$ & fiber coordinate in the fibration $\mathbb{C P}^{3} \rightarrow T \mathbb{C P}^{1}$ \\
\hline$\eta$ & $\$ 3.10$ & worldsheet field used for bosonization of $(U, W)$ \\
\hline & 4.4 & solution to the equation $\eta_{\alpha} \lambda^{\alpha}=1$ \\
\hline $\bar{\eta}^{\bar{z}}, \bar{\eta}^{\bar{w}}, \bar{\eta}_{A}$ & $\$ 3.7$ & B-model worldsheet fields \\
\hline$\Theta^{A}, \bar{\Theta}^{A}$ & $\$ 3.7$ & worldsheet fields \\
\hline$\vartheta_{z}, \vartheta_{w}, \vartheta_{A}$ & 33.7 & B-model worldsheet anti-commuting fields \\
\hline$\kappa$ & 4.4 & holomorphic component function on twistor space \\
\hline$\Lambda$ & 4.4 & arbitrary function on twistor space \\
\hline$\lambda^{\alpha}$ & 3.2 & twistor variable \\
\hline$\hat{\lambda}_{\dot{\alpha}}$ & $\$ 2.7$ & appears in the decomposition of null vectors \\
\hline$\mu_{\dot{\alpha}}$ & $\$ 3.2$ & twistor variable \\
\hline$\mu, \nu, \ldots$ & $\$ 2.7$ & spacetime indices $0, \ldots, 2$ or $0, \ldots, 3$ \\
\hline$\xi$ & 3.10 & worldsheet field used for bosonization of $(U, W)$ \\
\hline$\xi_{1}, \xi_{2}, \xi_{3}$ & 3.4 & projective coordinates on $W \mathbb{C P}^{1,1,2}$ \\
\hline & 4.6 & Stands for the worldsheet field $1 /\left(Z^{1} Z^{2}\right)$ \\
\hline$\pi, \pi^{\prime}$ & $\$ 3.2$ & projections from $D=4$ twistor space to $D=3$ mini-twistor space \\
\hline$\varpi$ & $\$ 4.4$ & holomorphic component function on twistor space \\
\hline$\rho^{z}, \rho^{w}, \rho^{A}$ & 3.7 & B-model worldsheet fields \\
\hline$\varrho, \widetilde{\varrho}$ & 8.4 & functions of $\lambda, \tilde{\lambda}$ appearing in the solution to Dirac's equation \\
\hline$\widehat{\varrho}, \widetilde{\widetilde{\varrho}}$ & 4.4 & twistor transforms of $\varrho, \widetilde{\varrho}$ \\
\hline$\sigma_{\alpha \beta}^{i}$ & 3.1 & $D=3$ Pauli matrices \\
\hline$S_{A B}$ & 4.4 & holomorphic component function on twistor space \\
\hline $\bar{\Sigma}$ & $\$ 3.4$ & curve in twistor space \\
\hline$\Sigma$ & $\$ 3.4$ & curve in mini-twistor space \\
\hline$v^{A}$ & 4.4 & holomorphic component function on twistor space \\
\hline$\Upsilon_{A}$ & 3.8 & Berkovits model fields dual to $\Theta^{A}$ \\
\hline$\phi$ & $\$ 3.10$ & worldsheet field used for bosonization of $(U, W)$ \\
\hline$\Phi^{I}$ & 3.1 & spacetime scalars \\
\hline$\chi_{A}, \tilde{\chi}^{A}$ & 4.4 & component fields of the B-model (functions of twistor space) \\
\hline$\chi_{\alpha}^{A}, \chi_{\alpha A}, \chi_{\alpha}^{a}$ & $\$ 3.1$ & $D=3$ fermions (functions of spacetime) \\
\hline $\begin{array}{l}\psi_{\alpha}^{A}, \bar{\psi}_{A}^{\alpha} \\
\Psi^{A}\end{array}$ & $\$ 3.1$ & $\begin{array}{l}D=4 \text { fermions (functions of spacetime) } \\
\text { fermionic coordinates in the B-model }\end{array}$ \\
\hline
\end{tabular}

Table 5: Various symbols used in the text (Greek letters). The "subsection" column is the place where the symbol first appeared with its given meaning. 